Portland State University

PDXScholar

$1-1-2011$

\title{
Self-Efficacy and Drinking with Friends: An Investigation into the Drinking Behaviors of Japanese College Students
}

Staci Jean Wendt

Portland State University

Follow this and additional works at: https://pdxscholar.library.pdx.edu/open_access_etds Let us know how access to this document benefits you.

\section{Recommended Citation}

Wendt, Staci Jean, "Self-Efficacy and Drinking with Friends: An Investigation into the Drinking Behaviors of Japanese College Students" (2011). Dissertations and Theses. Paper 293.

https://doi.org/10.15760/etd.293

This Dissertation is brought to you for free and open access. It has been accepted for inclusion in Dissertations and Theses by an authorized administrator of PDXScholar. Please contact us if we can make this document more accessible: pdxscholar@pdx.edu. 
Self-Efficacy and Drinking with Friends:

An Investigation into the Drinking Behaviors

of Japanese College Students

$$
\text { by }
$$

\section{Staci Jean Wendt}

A dissertation submitted in partial fulfillment of the requirements for the degree of

Doctor of Philosophy
in
Applied Psychology

Dissertation Committee: Cynthia D. Mohr, Chair

Eric Mankowski

Mo Wang

Liu-Qin Yang

Peter Collier

Portland State University

(C)2011 
Self-Efficacy and Drinking with Friends i

\begin{abstract}
Recent studies have documented an alarming rate of alcohol use in Japan (Eisenback-Stangl et al., 2005; Milne, 2003; Shimizu, 2000). Indeed, permissive social and cultural norms for alcohol use exist within Japanese culture (Shimizu, 1990, 2000). Japanese college-students may be at further risk due to their developmental time period, where increases in alcohol use are typically seen. Furthermore, drinking habits formed during this time period may be difficult to alter later in life (Frone, 2003). Thus, social, developmental, and cultural factors exist to influence drinking among Japanese college students. The purpose of the current study was to investigate the drinking behaviors of Japanese college students and possible proximal predictors of use. Specifically, given the importance of social relationships and interactions to interdependent cultures, such as Japan, the occurrence of negative social interactions may be influential in predicting subsequent drinking, as individuals may increase drinking in order to adhere to the social norms and to make amends. Hypothesis testing confirmed a significant and positive relationship between negative social events and drinking with others. Furthermore, the expected physical, social and emotional outcomes of alcohol consumption (alcohol outcome expectancies) have been shown to predict alcohol use among U.S. samples (e.g., Goldman, 1994), however, daily fluctuations in the desirability of alcohol outcome expectancies has not been previously investigated in a Japanese sample. Given the importance of fluctuations in desirability of alcohol outcome expectancies among U.S. samples
\end{abstract} (Armeli et al., 2005), this dissertation investigated daily fluctuations in the desirability 
of expected outcomes and alcohol use. Support for this relationship was found; on days with individuals experienced increases in the desirability of alcohol outcome expectancies, individuals drank more with others. Support for the hypothesis that increases in daily negative social events would predict increases in the desirability of alcohol outcome expectancies was not found. Finally, this dissertation investigated two types of self-efficacy (drinking refusal self-efficacy and social self-efficacy) as stable factors of drinking. Drinking refusal self-efficacy significantly and negatively predicted drinking with others; marginal support for drinking refusal self-efficacy as a moderator of the relationship between negative social events and drinking with others was found. Social self-efficacy significantly and positively predicted drinking with others. No support was found for social self-efficacy as a moderating variable in the relationship between negative social events and drinking with others. In sum, using data that was previously collected via daily process methodology, this dissertation investigated the relationships between daily negative social interactions, daily desirability of alcohol outcome expectancies, and drinking refusal and social selfefficacy as moderators of alcohol consumption. Support was found for five of the seven hypothesized relationships. 


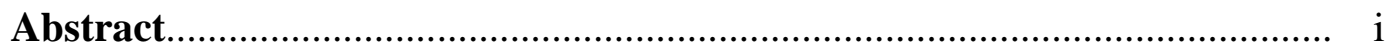

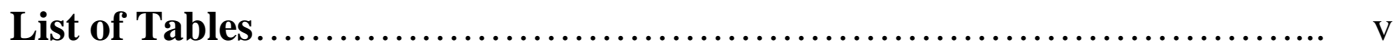

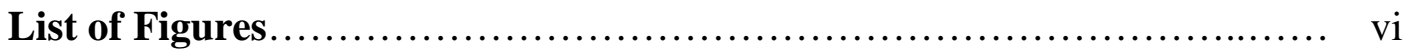

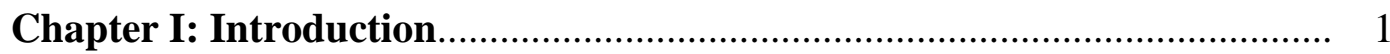

Chapter II: The Japanese Self and Negative Social Events........................... 13

The Japanese Self....................................................................................... 14

Social Events and Interactions and the Japanese Self......................................... 19

Chapter III: Alcohol Consumption in Japan and Among Japanese College Students.................................................... 22

College Student Drinking............................................................................. 30

Social Context of Drinking............................................. 32

Chapter IV: Alcohol Outcome Expectancy Desirability............................... 35

Predictors of Alcohol Consumption.................................................................. 35

Alcohol Outcome Expectancies....................................................................... 37

Alcohol Outcome Expectancy Desirability........................................................ 42

Chapter V: Daily Process Methodology .................................................... 46

Field Methods......................................................................................... 46

Daily Process Methodology........................................................................... 47

Types of Daily Process Protocols....................................................................... 50

Chapter VI: Self-Efficacy ................................................................... 54

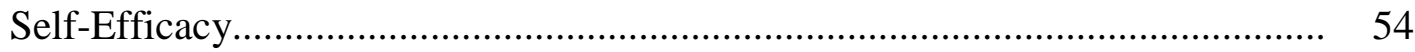

Drinking Refusal Self-Efficacy.................................................................... 60

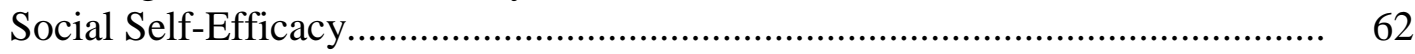

Chapter VII: Development of Research Questions and Hypotheses.............. 66

The Proposed Study ..................................................................................... 67

Hypotheses and Research Questions........................................................... 68

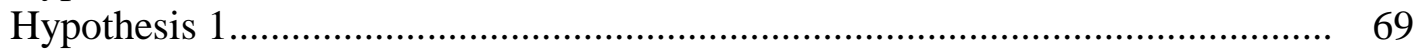

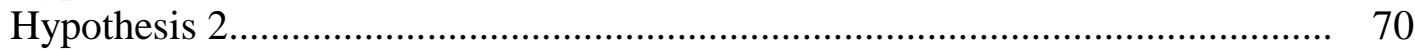

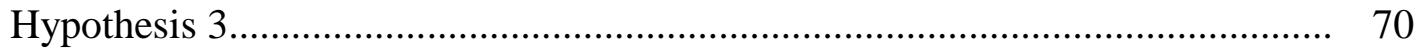

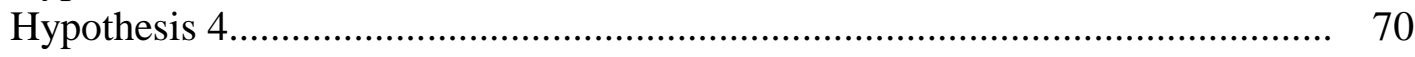

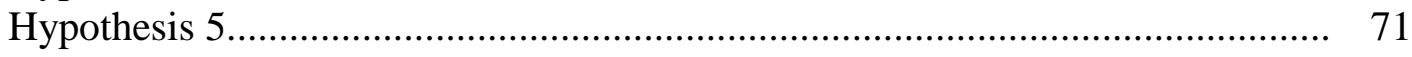

Research Question 1........................................................................... 72

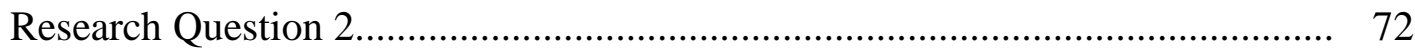


Chapter VIII: Methods......................................................................... 73

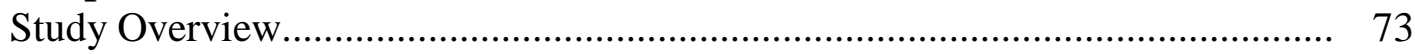

Procedure.............................................................. 73

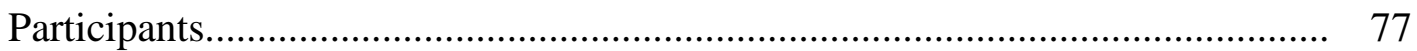

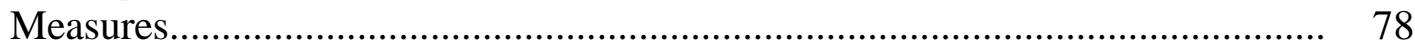

Initial Assessment Measures................................................................. 78

Daily Interview Measures.............................................................. 80

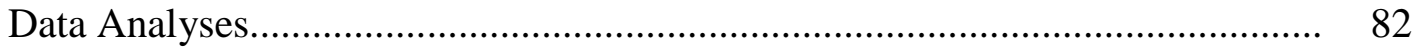

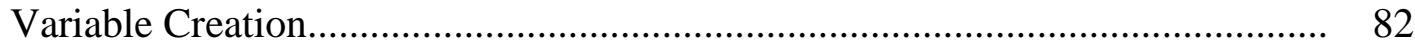

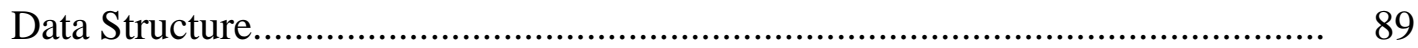

Chapter IX: Results...................................................................... 91

Descriptive Information...................................................................... 91

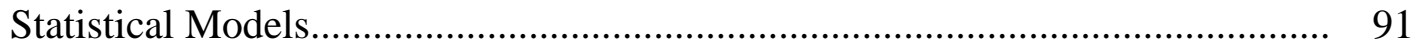

Hypothesis Testing .............................................................................. 93

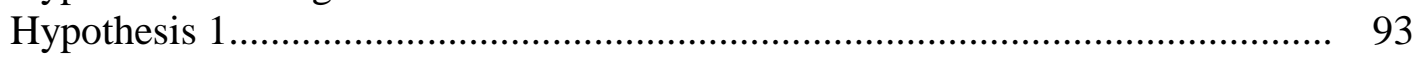

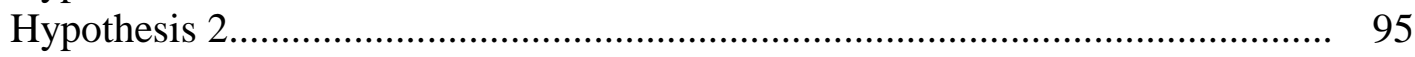

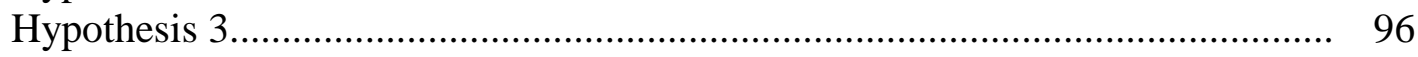

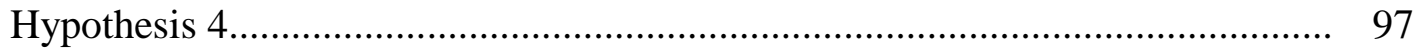

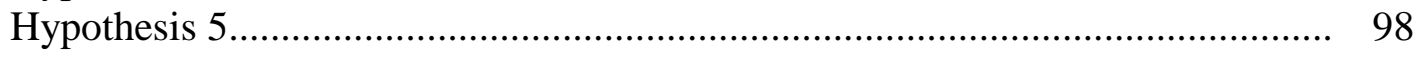

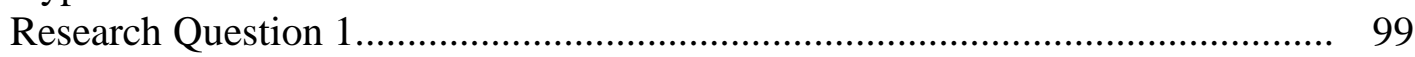

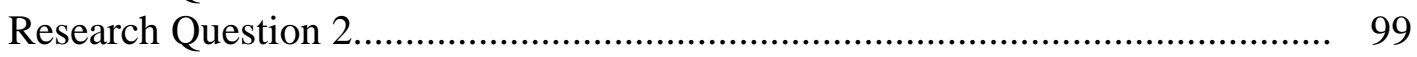

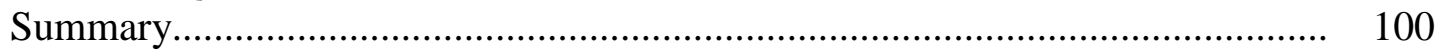

Chapter X: Discussion..................................................................... 104

Negative Social Events and Drinking with Others........................................ 106

Alcohol Outcome Expectancy Desirability................................................ 108

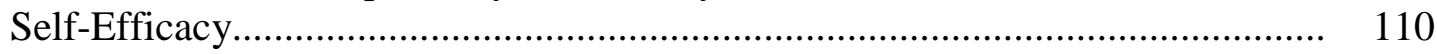

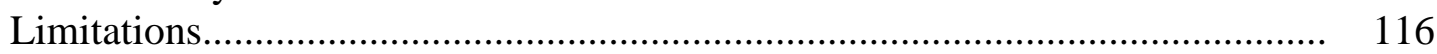

Theoretical and Practical Implications.................................................... 120

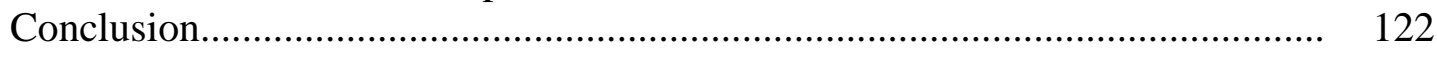

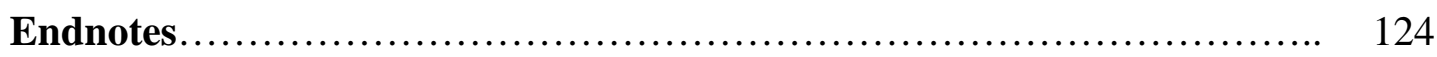

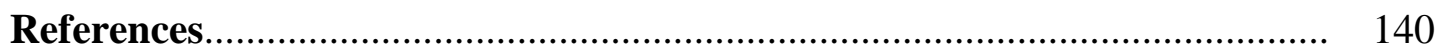

Appendix A. Initial Assessment Measures................................................. 167

Appendix B. Daily Interview Measures..................................... 169 
Table 1. Between-person correlations.......................................... 131

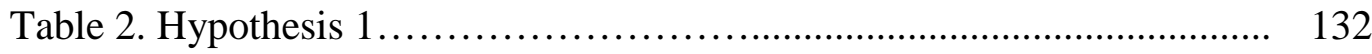

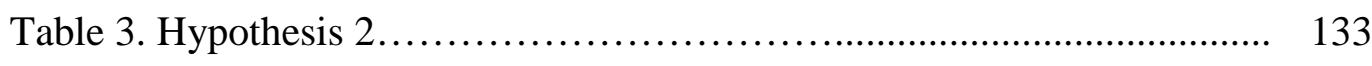

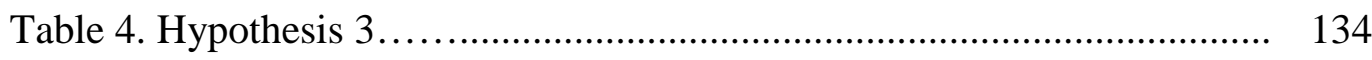

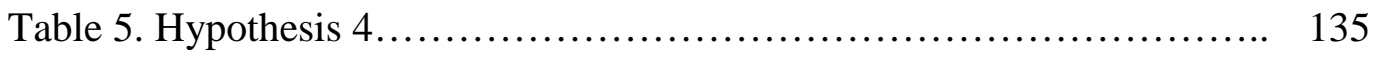

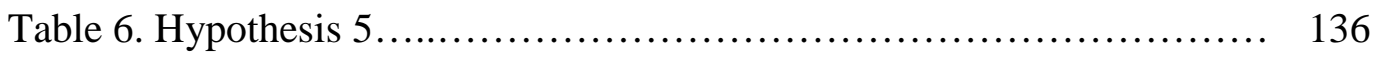

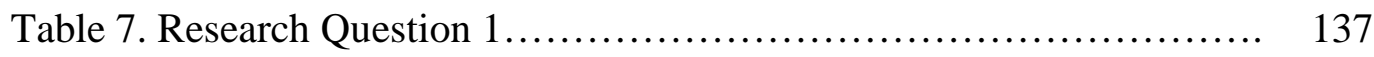

Table 8. Research Question 2 ................................... 138

Table 9. Hypothesis testing results............................... 139 


\section{List of Figures}

Figure 1. Proposed theoretical model of alcohol use...................................... 125

Figure 2. Predictors of alcohol consumption................................................ 126

Figure 3. Transactional model of stress and coping..................................... 127

Figure 4. Key measurements................................................................. 128

Figure 5. Daily interview protocol................................... 129

Figure 6. Example of missing data................................... 130 


\section{CHAPTER I}

\section{Introduction}

Alcohol use in Japan is dramatically increasing (Higuchi, Matsushita, \& Osaki, 2006). Results from a 2001 national survey of drinking found that $91 \%$ of men and $77 \%$ of women report being current drinkers (Bond, Greenfield, Roberts, \& Korcha, 2010) and monthly binge-drinking rates as high as 37\% among men (Grittner, Kuntsche, \& Bloomfield, 2010), compared to data from 1968 where only $76 \%$ of men and $19 \%$ of women reported being current drinkers (Higuchi et al., 2006). Following World War II, the norms surrounding alcohol use have become increasingly permissive (Shimizu, 1990); for example, it is common to find vending machines on street corners that sell alcohol. Further, public displays of drunkenness, especially among businessmen, are widely accepted and permitted (Shimizu, 1990). The high levels of consumption are of concern to health researchers as the Japanese may have particular biological predispositions to negative health effects of alcohol consumptions, such as cancer and liver disease (e.g., Luczak, Glatt, \& Wall, 2006).

Given the prevalence of alcohol consumption in Japan, it is important to understand possible cultural and contextual antecedents of alcohol use, in order to inform interventions to reduce use. First, it is important to understand the possible ways in which culture influences consumption. Japanese culture is considered a collectivist or interdependent culture (Benedict, 1946; Markus \& Kitayama, 1991). The Japanese self is embedded within a social framework, wherein social relationships and connections are considered fundamental to the self (e.g. Fiske, 1992; Kitayama \& 
Uchida, 2004). Research has shown that the Japanese are reactive to the emotional experiences and expressions of others, in addition to their own experiences (Kitayama, Markus, \& Kurokawa, 2000). Negative social interactions are particularly distressing to Japanese individuals, as they indicate a failure in the interdependent task to properly maintain relationships, as indicated by the experience of shame (Mesquita \& Karasawa, 2002). Specifically, Mesquita and Karasawa (2002) found that, compared to U.S. college students, Japanese college students reported greater distress in response to thinking about negative social interactions with their friends. The response to negative social interactions is shame (Benedict, 1946).

Shame is a critical emotion for the Japanese, as it reflects the failure to maintain or foster relationships; for the Japanese, social connections are central to well-being (Markus \& Kitayama, 1991; Mesquita \& Leu, 2007). Compared to more independent cultures, the Japanese are more strongly affected by the emotional expressions of others (Scollon, Diener, Oishi, \& Biswas-Diener, 2004). For the Japanese, making repairs often includes the willingness to conform to others' behaviors or desires, or to alter one's own behaviors to match those of the larger group (Morling, Kitayama, \& Miyamoto, 2002; Mesquita \& Leu, 2007). In order to make reparations for these negative interpersonal interactions, the Japanese seek out others and attempt to repair these relationships (Mascolo, Fischer, \& Li, 2003).

One context where individuals may be able to make amends for negative interactions is in drinking with friends. Indeed, strong, permissive social norms for drinking have been documented in Japan (Shimizu, 1990, 2000). In Japan, drinking 
with friends is seen as a method to form and affirm connections with one's social group (Shimizu, 2000). Among a society that normally follows a strict, hierarchical structure of social relationships, when consuming alcohol, the social rules are relaxed (e.g., Allison, 1994; DeMente, 1995). Thus, drinking with friends is one way that Japanese individuals may repair relationships, specifically in response to a prior negative social interaction.

In addition to the strong social norms for drinking, researchers have documented several negative health consequences of alcohol consumption, including liver disease (Parrish, Higuchi, Muramatsu, Stinson, \& Harford, 1991), esophageal cancer (Parrish, Higuchi, \& Lucas, 1993), and suicide (Akechi, Iwasaki, Uchitomi, \& Tsugane, 2006). Japanese individuals may be at increased risk to these negative health consequences due to the prevalence of the ALDH2 genotype (Takeshita \& Morimoto, 1999), which makes the digestion of alcohol more harmful to the body. Moreover, the increases in quantity and frequency of alcohol consumption typically seen among college students (Baer \& Carney, 1993) may put college students at an additional risk for the development of alcohol-related problems and the physical health consequences of drinking, as habits formed during this developmental time period may continue into adulthood (Babor et al., 1992). Such increases may be due to the developmental time period known as emerging adulthood, when students often experience more personal freedom and less social control, compared to earlier developmental time periods (Arnett, 2000). Further, given the strong social influence within Japanese culture, certain social contexts may be riskier for young Japanese adults, compared to U.S. 
college students, in terms of pressure to consume alcohol (Higuchi, Matsushita, Muramatsu, Murayama, \& Hayashida, 1996).

Thus, drinking behavior among Japanese college students is multifaceted, including developmental, cultural, and contextual influences. Moreover, given the high levels of alcohol use in Japan, and the documented consequences of such use, it is important to understand possible pathways to alcohol consumption. The experience of negative interpersonal events may be an important antecedent to alcohol consumption within Japanese culture. Therefore, in response to negative interactions, Japanese individuals may be more likely to consume alcohol with their friends, instead of in more solitary contexts. Thus, the drinking context may differ for Japanese drinkers, compared to U.S. drinkers, suggesting that a study investigating the distinct predictors of alcohol consumption for Japanese drinkers is warranted.

Indeed, Cooper (1994) distinguished solitary from social contexts for drinking; drinking in solitary contexts was related to drinking-to-cope behaviors and was more likely to lead to the development of alcohol-related problems, whereas drinking in social contexts was more related to social enhancement. Social drinking in response to negative social interactions may be a distinct behavior that is characteristic of drinking among the Japanese. It is important to investigate a theoretical model of alcohol consumption that accounts for the unique impact of negative social interactions, the socio-cultural norms, and differences in perceived alcohol outcome expectancies that may contribute to drinking among the Japanese (see Figure 1). 
The strong beliefs about the socially enhancing benefits of alcohol consumption may be an important antecedent to drinking. That is, in a society with high levels of consumption and strong social norms for drinking, individuals may hold alcohol outcome expectancies that alcohol lubricates social interactions. Alcohol outcome expectancies are the beliefs regarding physical, emotional, and social effects of alcohol consumption (Marlatt \& Rosenhow, 1980). Alcohol outcome expectancies develop over time and are influenced by cultural, biological, and social influences, in addition to an individual's direct experience with alcohol (MacCorquodale \& Meehl, 1953). Alcohol expectancies have been described as one of the strongest predictors of alcohol use (Goldman, 1994), predicting quantity (Carey, 1995), frequency (Fromme, Stroot, \& Kaplan, 1993), initiation and maintenance of alcohol consumption (Brown, Christiansen, \& Goldman, 1987).

Given the use of alcohol as a social facilitator in Japan (i.e. Shimizu, 2000), Japanese drinkers may have positive alcohol outcome expectancies related to feeling more connected to the social group or relieving tension. These positive alcohol outcome expectancies, or the beliefs about the effects of alcohol, have been shown to predict frequency and quantity of drinking for a variety of samples (e.g., Goldman, Del Boca, \& Darkes, 1999). Thus, in addition to cultural and contextual norms for drinking, alcohol outcome expectancies are important antecedents of drinking. Embedded within the alcohol outcome expectancies is the desirability of these outcomes (e.g., Leigh, 1989). Desirability is defined as the degree to which an individual wants to achieve an expected outcome. Whereas alcohol outcome 
expectancies are developed over time and remain relatively stable, fluctuations occur in the desirability of these outcomes (Armeli et al., 2005). For example, an individual may expect to become more assertive when she/he drinks alcohol, however, if she/he would prefer to relax, the desirability of this expected outcome is low, and it would be expected that the individual would not consume alcohol. Specifically in response to the experience of negative social events, alcohol outcome expectancies related to social enhancement and bonding with friends may be more desirable, although this relationship has not previously been studied.

Whereas negative social events may be associated with higher levels of drinking, there may be protective factors, such as self-efficacy that limit or reduce drinking (Figure 1). Self-efficacy has been identified in a number of health models as a major contributing factor to health behaviors (e.g., Theory of the Planned Behavior, Ajzen, 1991; Health Belief Model, Rosenstock, 1966). Generally, self-efficacy has been shown to protect individuals against the negative effects of stressful events (e.g., Bandura, 1992). Specifically, previous research has identified a type of self-efficacy, drinking refusal self-efficacy, or the belief about one's ability to abstain from drinking in certain contexts, as an important factor in determining drinking (Oei, Fergusson, \& Lee, 1998), and may be particularly important to Japanese college students who are confronted with strong social pressures to consume alcohol. Furthermore, social selfefficacy, or one's beliefs about her/his ability to initiate and maintain social relationships, has been shown to be a between-person predictor of health behavior for cultures where maintaining relationships is crucial to well-being (Gong \& Fan, 2006). 
Therefore, social and drinking refusal self-efficacy may play an important role in the association between the experience of negative events and drinking.

Given the variation in the number and type of occurrences of daily interactions, information regarding these social interactions should be collected daily. Likewise, given the fluctuations in the desirability of alcohol outcome expectancies, researchers should obtain reports of desirability as close to real-time as possible. The advent of daily process methodology has been critical to the understanding of within-person variation in interpersonal interactions and drinking. Daily process methodology is characterized as collecting data on a daily basis using various types of recording schedules (e.g. Reis \& Gable, 2000). Daily process methodology allows researchers to establish a temporal sequence between events and behaviors (such as negative social events and drinking) to be established, as data is collected daily over several weeks. Additionally, daily process methodology is useful for collecting data that is vulnerable to retrospective bias, such as emotionally relevant information (Thomas \& Diener, 1990) and contingencies between drinking behavior, such as mood (Carney, Tennen, Affleck, Del Boca, \& Kranzler, 1998). Indeed, retrospective reports of mood may show truncated variability (Robinson \& Clore, 2002) or may be influenced by an individual's current mood (Snyder \& White, 1982). In conjunction with multi-level modeling, daily process data allows for investigations of intra-individual variations in daily events and alcohol consumption and inter-individual factors such as selfefficacy. In other words, daily process data allows researchers to examine within- and between-person associations, such as those proposed in Figure 1. This dissertation will 
use data collected daily to further examine the proposed model of alcohol consumption.

Overview of the Proposal

Using daily process methodology, this dissertation will investigate the relationships between negative social events, alcohol outcome expectancy desirabilities, drinking refusal and social self-efficacy, and alcohol consumption among a Japanese college student sample. Given the social nature of drinking among this population, and the strong influences for drinking in social contexts (e.g. Shimizu, 1990, 2000), this dissertation will look specifically at drinking with others. In particular, this dissertation will examine whether Japanese students drink more with others on days with greater negative social events relative to days with fewer negative social events, as highlighted in the proposed theoretical model in Figure 1. In line with models of shame-related drinking (e.g., Dearing, Stuewig, \& Tangney 2005; Mohr et al., 2008), it is hypothesized that students will drink more with others on days characterized by greater negative social interactions compared to days with fewer negative social interactions, as negative social interactions are likely to invoke a need to make amends for negative social interactions (Benedict, 1946; Morling et al., 2002; Mascolo et al., 2003; Mesquita \& Leu, 2007). Second, this dissertation will investigate whether daily negative social events predict daily alcohol outcome expectancy desirability, such that expectancies related to relationship maintenance and tension reduction are more desirable on days characterized by negative social events, relative to days with fewer negative social events. Third, it is hypothesized that drinking with 
others will be greater on days when alcohol outcome expectancies are rated as more desirable, compared to days with lower rated desirability. Fourth, this dissertation will examine drinking refusal self-efficacy and social self-efficacy as cross-level moderators of negative social events and drinking with others. Specifically, based on existing research that demonstrates an inverse relationship between drinking refusal self-efficacy and consumption (Oei, Hasking, \& Phillips, 2007), it is hypothesized that negative social events will have less of an effect on drinking for individuals who have high perceived drinking refusal self-efficacy, compared to those with low drinking refusal self-efficacy. Finally, this dissertation will investigate the relationship between social self-efficacy, negative social events and drinking. Specifically, is social selfefficacy a between-person moderator of the relationship between negative social events and drinking? This research question is a non-directional hypothesis as it is possible that drinking with others, as it is seen as a normative behavior, may benefit relationships and thus be a demonstration of greater social self-efficacy. Conversely, it is reasonable that individuals with high social self-efficacy may be less affected by negative social events, and therefore be less inclined to drink with others as a way to make amends.

The significant contributions of this work include identifying within-person variation in drinking among Japanese college students. This work will provide a thorough description of negatively-appraised social events. This is a significant contribution, as the current study will look at appraisals of events, thus reducing researcher bias in regards to what is considered a negative compared to a positive 
event. This will have implications for future studies, as it is likely that there are crosscultural differences in event appraisals, and few studies have investigated event appraisals for research samples outside of the U.S. Further, this study has crosscultural implications, as it will be the first study to examine alcohol outcome expectancy desirability and alcohol consumption among a Japanese sample. This study will add to the self-efficacy literature, as it will examine two specific types of selfefficacy and their relationship to alcohol consumption. Finally, this study will provide a unique investigation into the cultural, contextual and individual components of alcohol consumption.

This research was part of a larger study investigating cross-cultural adjustment and health-related outcomes among a sample of Japanese students. Participants were students from Waseda University. A portion of the sample was international students studying at Portland State University, the remainder of the sample was students studying in Japan. The project was directed by Mo Wang, Ph.D., and Cynthia D. Mohr, Ph.D., and funded by the Medical Research Foundation of Oregon. I assisted Drs. Wang and Mohr by serving as Project Manager and was involved in all aspects of the project. This project examined daily health behaviors, such as alcohol, caffeine, and nicotine consumption, as well as daily interpersonal interactions, among Japanese college students studying in the United States and Japan. The purpose of the study was to investigate cross-cultural adjustment as a moderator of interpersonal interactions and alcohol consumption. The 57 participants accessed a web survey each afternoon between $3-8 \mathrm{pm}$ for 30 days. The web survey instrument probed positive and negative 
interpersonal exchanges and alcohol use. Using this approach, I will conduct a secondary analysis of this data to investigate how daily negative interactions predict subsequent drinking behavior.

Using hierarchical linear modeling (HLM, v6.0; Raudenbush, Bryk, Cheong, \& Congdon, 2000) to analyze this data, this dissertation will investigate the relationships between negative social events, alcohol outcome expectancy desirabilities, drinking refusal and social self-efficacy and alcohol consumption among a Japanese college student sample (Figure 1). The purpose of this dissertation is to determine the relationship between negative social events, self-efficacy, and drinking among a Japanese sample.

The proposal proceeds as follows. First, in Chapter II, I will provide a review of the Japanese self and how this interdependent self relates to others. This chapter will serve to situate the remainder of the proposal within the context of the Japanese self. Chapter II will also discuss negative social events and in Chapter III I will discuss alcohol consumption among the Japanese population, generally, and college-students, specifically. Following the proposed model of alcohol consumption (Figure 1), in Chapter IV, I will provide a more detailed review of alcohol outcome expectancies and desirability, which are situated in the larger context of the drinking literature. In Chapter IV, I will discuss daily process methodology and the advantages of collecting data using this methodology. Next, in Chapter VI, I will review the broad concept of self-efficacy as well as the specific types of self-efficacy that will be investigated in the current project: drinking refusal self-efficacy and social self-efficacy. In Chapter 
VII, I will discuss the current study, hypotheses, and research questions. In Chapter VIII, I will present the proposed methods and analyses. Finally, in Chapter IX, I will discuss the implications and limitations of the proposed study. 


\section{CHAPTER II}

The Japanese Self and Negative Social Events

The purpose of this chapter is to describe the Japanese self. After explaining the larger interdependent cultural model, I will discuss how negative interactions influence behavior for an interdependent culture, such as Japan. Finally, I will discuss the interaction of the interdependent cultural self and the experience of negative social events, which in turn relate to alcohol consumption. It is important to recognize these cultural differences in order to understand why motivational models of alcohol use, as will be discussed in the remainder of this dissertation, may differ for these populations, based on the differences in emotional experiences. Thus, this chapter will serve as a foundation for constructs and behavior discussed in later chapters of this dissertation.

Culture is the shared beliefs and norms shared by a population of people who share a common language and interact within the same geographical space within the same historical period (Triandis, 1989). As Triandis (1989) said, "Culture is to society what memory is to the person." Culture influences the way individuals think about the self, and interact in social situations (Triandis, 1989). Culture includes all aspects of a society, including economic, political and educational systems, social structures, and language (Triandis, 1989). Thus, understanding the cultural self is an important foundation with which to understand alcohol consumption; cultural views of the self and of one's relationship influence daily behavior and social institutions (e.g., Kitayama \& Markus, 1999; Kitayama \& Park, 2007). 


\section{The Japanese Self}

Social scientists have documented cultural differences in psychological processes (Fiske, Kitayama, Markus, \& Nisbett, 1998; Markus \& Kitayama, 1991; Triandis, 1989). According to cultural psychological models, core cultural ideas, such as what is good, what is moral, and what constitutes the self, have direct and indirect influences on psychological processes and action (Fiske et al., 1998; Kitayama \& Markus, 1999). This collective reality influences the customs, norms, and systemic reality, as well as the individual's reality. Individuals live within differing sociocultural contexts, and feelings and behaviors must be considered within these cultural models (Mesquita \& Albert, 2007). Thus, cultural influences are a fundamental root of individual behavior.

The way an individual perceives her/himself in relation to the surrounding environment comprises the cultural view of the self. Researchers commonly separate these viewpoints into independent or interdependent categories ${ }^{1}{ }^{1}$. In turn, these cultural views of the self compose behavior; beliefs and behaviors that are congruent with the dominant views of the self and relationships are likely to continue and strengthen over time (Kitayama \& Park, 2007). Cultural influences can be seen in public artifacts, such as advertising, socially shared meanings, such as the definition of happiness, and socially constructed situations, such as those that elicit increased relatedness with one's social network (Kitayama \& Park, 2007); they serve to reflect and reinforce a culture. Within a society, individuals not only act in ways that promote the dominant culture, but also the social situations in which they interact encourage 
behavior congruent with the culture (e.g. Lebra, 1976; Markus \& Kitayama, 1991;

Triandis, 1989). In the following sections, I will provide examples of public artifacts, socially shared meanings, and socially constructed behavior to demonstrate the interdependent culture.

The independent and interdependent cultural models have various influences on emotional, motivational, and cognitive processes (Markus \& Kitayama, 1991). Western cultures, mainly the U.S. and Britain are considered more independent compared to the more interdependent cultures, such as Japan or China (Triandis, 1989). The right to make one's own choices is characteristic of more individualistic cultures. The self is perceived as separate from others; the self is known by one's independence from others (Fiske et al., 1998; Markus \& Kitayama, 1991; Triandis, 1989). Autonomy and independence are the main goals of this model. The individualist model of the self has been the implicit model/framework used in Western psychology until recent years. However, a second model, the interdependent model, also exists.

The interdependent model is prevalent in cultures such as Japan (Benedict, 1946; Markus \& Kitayama, 1991). In this model, the self is seen as interconnected with others and the environment. This model emphasizes belongingness and social cohesion. The interdependent model is reinforced through parenting practices that accentuate conformity, obedience and reliability (Triandis, 1989). The self is known by one's relationship to others. In this model, the self works to adjust to others' expectations and the larger group ideals. The major task of the interdependent self is to 
maintain relationships by adjusting oneself within important relationships, engaging in appropriate, norm-driven actions, and promoting larger group goals. Thus, relational goals take precedence over personal goals (Kitayama, Markus, \& Matsumoto, 1995; Heine, Kitayama, Lehman, Takata, Ide, \& Leung, 2001).

In an analysis of public artifacts of culture, Markus, Uchida, Omoregie, Townsend and Kitayama (2006) analyzed media coverage of the 2000 and 2002 Olympics in Japan (classified as an interdependent culture) and the U.S. (classified as an independent culture). Their analysis showed that in American media, athletes were described in a way that highlighted her/his internal attributes (i.e., individual talent, effort, or perseverance). In the American coverage, very little attention was given to the athlete's home-life or upbringing. When athletes won events, credit was given to the individual athlete, not to the coach or the team. In contrast, the Japanese media described athletes in terms of her/his background, her/his support system, and/or her/his team effort.

In a second example of public artifacts projecting cultural models, Kim and Markus (1999) compared advertisements in South Korea (classified as an interdependent culture) and the U.S. In South Korea, advertisements often used conformity appeals that promoted group harmony, whereas the American advertisements highlighted individual differences and uniqueness. The American advertisements were more likely to include appeals that emphasized freedom of choice over appeals of conformity. These examples of public artifacts demonstrate the 
emphasis interdependent cultures place the important of conforming to the social norm, whereas independent cultures emphasize uniqueness and individualism.

Lebra (1976) noted that social interactions and relationships were of central concern to Japanese individuals. Indeed, Japanese make more internal adjustments to adapt to social relationships and the larger social environment, are more focused on maintaining relationships and avoiding negative perceptions of the self, compared to Americans (Markus \& Kitayama, 1991; Heine et al., 2001; Karasawa, 2001; Mesquita \& Karasawa, 2002). The Japanese self is more likely to conform to one's behavior to match those of the group around her/him, compared to Americans. Similarly, in response to relational conflict, the Japanese are more likely to make internal changes, as opposed to expecting their partner to change their behavior. The Japanese self strives for harmonious relationships and relational closeness is central to well-being (e.g., Markus, Kitayama, \& Heiman, 1997; Oishi \& Diener, 2001). Social motivations are oriented toward fitting in to the interpersonal context and maintaining relationships (Kwan, Bond, \& Singelis, 1997; Kitayama \& Markus, 2000; Kitayama, Markus, \& Kurokawa, 2000).

For the Japanese, giri is key to understanding social obligations. Although giri does not have a direct English translation, Davies and Ikeno (2002) defined giri as a constellation of related meanings that "define moral principles or duty, the rules one has to obey in social relationships, and the behavior one is obliged to follow or that must be done against one's will (p.95)." To go against giri is to fail in social obligations. For example, if an individual invites a friend out for a drink, and the 
friend does not want to go, if the friend declines, they are not adhering to their social obligation. Thus, the friend will accept the invitation, despite preferring to not attend the event (Davies \& Ikeno, 2002).

Furthermore, the hierarchical or vertical structure of social relationships in Japan dictates that reverence be paid to one's elders or superiors. The younger Japanese individual must acquiesce to the requests of the older or superior individual (Shimizu, 1990; Davies \& Ikeno, 2002). Failure to do so results in dishonoring one's social group.

For the interdependent self, the successes and failures of members within one's social group reflect on the individual (Benedict, 1946). For this reason, an individual's actions, if they are not in accordance with group norms, or reflect poorly on the group, are reprimanded in a way that causes shame, or negative feelings about the self. Individuals in an interdependent culture strive to adhere to social norms and to act in ways that benefit the larger group (Kitayama, Markus, \& Matsumoto, 1995).

In sum, for more interdependent variations of the self, the self is defined in terms of her/his relationship to her/his family and society; the self is not detached from these relationships (Sampson, 1988). The interdependent self is complex and is defined by the social obligations, norms and rituals of the larger in-group (Triandis, 1989). That is, belongingness, dependency, empathy, and one's place in a group are of constant concern for the interdependent self (Markus \& Kitayama, 1991). This interdependent construal of the self directly influences the experience of emotions (Markus \& Kitayama, 1991; Suh, Diener, Oishi, \& Triandis, 1988; Triandis, 1989). 
Social Events and Interactions and the Japanese Self

Japanese individuals are focused on self-improvement and the fulfillment of relational obligations (e.g. Mesquita \& Karasawa, 2004; Karasawa, 2001). As previously discussed, individuals strive for relational harmony and maintenance. In the interdependent cultural model, the core social motive is to gain acceptance and to avoid rejection (Fiske, 2004). Moreover, Mesquita and Leu (2007) found that when Japanese participants reported their own emotional experience, the perceived emotional experiences of others were more central to the individual's reported emotional experience than the individual's actual emotional experience. In contrast to Americans, who seek out situations that are likely to elicit positive affect, the Japanese seek out situations that enable them to meet social standards, rather than increase positive affect (Scollon et al., 2004).

Thus, when negative social events or interactions (e.g. embarrassing oneself in front of others, having a disagreement with friends) occur, the interdependent individual seeks to self-improve by seeking out others and attempting to make amends (Kitayama \& Markus, 2000; Suh et al., 1998). That is, Japanese individuals seek to reduce social distance, make changes to ones self to fit in with important others, or to submit to others (Markus et al., 1997).

In interdependent contexts, individuals attempt to restore relationships in response to experiencing negative social interactions, such as letting down one's group or getting into an argument with a friend (Markus \& Kitayama, 1991; Mesquita \& Karasawa, 2002). These types of negative social interactions isolate the individual 
from the larger group, which indicates a failure to preserve and cultivate relationships, a motive central to the interdependent self. The experience of negative social interactions or failing at interdependent tasks is typically followed by public apologies, and declarations to change the unworthy action (Mesquita, 2001). Thus, in the interdependent model, individuals choose to engage themselves within their social network (Kitayama et al., 2000; Suh et al., 1998).

For the Japanese, action in response to negative events is self-adjustment (Kitayama, Duffy, \& Uchida, 2007); happiness and well-being are embedded in the maintenance of social relationships. For example, Suh et al. (1998) found that behaviors that include adapting to social norms and fulfilling relational obligations increased happiness among East Asians (including the Japanese). Miyamoto and Kitayama (2002) found that happiness was conceptualized as feeling harmonious in one's relationships. Uchida and Kitayama (2009) asked American and Japanese college students to write about different aspects of unhappiness or happiness, and then to rate each characteristic of the event as either desirable or undesirable. The results showed that, for the Japanese students, happiness was more strongly associated with social harmony than with personal achievement. Additionally, Japanese students indicated that restoring harmony and interdependence in the social situation by fitting in with the relative norms was a way to cope with unhappiness. Kitayama et al. (2000) also found an association between a sense of well-being and acting in harmony with social norms and social scripts for Japanese students. 
One way that individuals in this relatively interdependent context may choose to make reparations to members of their social network, after the occurrence of negative social events is to consume alcohol with friends. Specifically, as will be discussed in Chapter 3, drinking with friends is seen as an interpersonal experience (Shimizu, 1990, 2000). Furthermore, as previously discussed, when negative social events have occurred, the Japanese seek out friends in order to make amends, and attempt to regulate behavior to match that of the larger social group. Therefore, if the larger group is consuming alcohol, it is reasonable to predict that the individual will also consume alcohol. Thus, following the Kitayama and Park's (2007) framework for understanding the reciprocal relationship between cultural views of the self and socially constructed situations, drinking with others as a method of amending negative social interactions may be a situation where pressures to conform are highest. As I will discuss in the following chapter, strong social norms for drinking exist within Japanese culture, and the social context for drinking is a particular environment that highlights conformity to social norms for drinking. 


\section{Chapter III}

Alcohol Consumption in Japan and Among Japanese College Students

As discussed in Chapter II, within an interdependent culture, the pressure to conform to the larger in-group norms and beliefs is stronger, compared to an independent culture (Suh et al., 1998). Moreover, within an interdependent culture, social behavior is a function of the in-group norms (Markus \& Kitayama, 1991). Interpersonal conflict is relatively avoided; when conflict occurs, it causes emotions such as shame (Markus \& Kitayama, 1991). Consuming alcohol with friends is one way to atone for the previous negative social interactions and strengthen relationships. Thus, the experience of negative social interactions may be detrimental to Japanese individuals as these events may predict heavy subsequent alcohol use. In this chapter, I will discuss the current level of alcohol use in Japan and possible facilitators to alcohol use. I will also review the literature on alcohol use among Japanese college students, as individuals within this developmental time period may be at increased risk for the development of alcohol-related problems. Similar to Chapter II, where I discussed the Japanese self, Chapter III will serve as a building block for the remainder of this dissertation. Moreover, in the proposed study, alcohol consumption will be the outcome variable of interest.

\section{Alcohol Consumption in Japan}

Existing studies of alcohol consumption are primarily limited in their focus on American populations, which, as previously discussed, may have limited generalizability to more interdependent cultures, where social influences are more 
strongly related to behavior (e.g. Markus \& Kitayama, 1991). Specifically, extensive research examining the causal factors of alcohol consumption among the Japanese population does not exist. For example, the first nationally representative survey of excessive drinking was conducted in 2001 (Milne, 2003), indicating a rather nascent investigation into alcohol use. However, studies have begun to document that this population is at risk, as alcohol use is high. In fact, Milne (2003) wrote that Japan was in the midst of an alcoholism crisis, due to the dramatic increases in levels of alcohol consumption during the past 30 years (Higuchi et al., 2006; Kitano, Chi, Rhee, Law, \& Lubben, 1992).

In a review of the prevalence of alcohol consumption in Japan, Higuchi et al. (2006) outlined the following historical developments. The Japanese have used alcohol in Shinto rituals and other events throughout history. Prior to World War II, alcohol use was used primarily for religious or ceremonial purposes, and was often consumed in the form of rice wine, which was common in villages (due to the local agriculture). Thus, alcohol use was limited. However, following WWII, alcohol use increased considerably. Higuchi et al. (2006) theorize that alcohol use has increased due to the rapid development of Japan and economic prosperity, thereby making alcohol more accessible and encouraging a more liberal view of alcohol consumption. There has also been a dramatic change in the types of alcohol consumed; beer and "alcopops" are quite common among drink choices. Alcopops are flavored spirits, sold in a can or a bottle and have an alcohol content around 10\%. Finally, Higuchi et al. (2006) attribute the increase in alcohol consumption to the diversification of the 
alcohol drinking population. Alcohol use among females, youth and the elderly has increased (Higuchi \& Kono, 1994); more than $60 \%$ of junior high aged and $80 \%$ of high school aged students have reported consuming alcohol (Higuchi et al., 2006).

Indeed, in a seminal paper on drinking culture in Japan, Shimizu (1990) proposed the concept of an alcoholic social system. This social system links the relationship between Japanese drinking culture and Japanese social structure. Specifically, Shimizu outlined four tenets of this alcoholic social system: 1) a general acceptance of drinking and toward drunkenness shared by group members; 2) that social relationships are organized around alcohol; 3) structural vulnerability against the negative effects of alcohol consumption; 4) the mechanism of simultaneous integration of permissiveness and control. Shimizu (1990) noted that these characteristics were only applicable to men at the time, but that drinking among women was becoming more acceptable. I will return to the rise in consumption among women later in this chapter. In fact, Shimizu posited that it would be difficult for Japanese society to function without alcohol. According to a typology of drinking cultures outlined by Pittman (1967), Japan is considered a permissive or overpermissively drinking culture, meaning that drunkenness and occasional pathological forms of drinking, in addition to normal drinking are accepted. According to Shimizu (1990), nationwide, more than $45 \%$ of the male population consumes alcohol on 4 or more days a week. This daily, nighttime drinking is called banshaku and occurs only at night, after work duties are completed. 
The most recent estimates of costs of alcohol use in Japan were at $\$ 60$ billion (U.S. dollars) in 1987 (Nakamura, Tanaka, \& Takano, 1993). In 1987, this accounted for 1.9\% of Japan's Gross Domestic Product (GDP; Thavorncharoensap, Teerawattananon, Yothasamut, Lertpitakpong, \& Chaikledkaew, 2009). This report is the most current estimate of alcohol-use related costs in Japan. Results from the GENACIS (Gender, Alcohol, and Culture: An International Study; Bloomfield et al., 2005) project, which documents multi-national alcohol use, show that, among the 23 countries $^{2}$ included in the study, Japan ranked sixth in the number of current drinkers (approximately $80 \%$ of women, $95 \%$ of men). For comparison, the US ranked $18^{\text {th }}$ (approximately 65\% of women, $75 \%$ of men); Austria ranked first (approximately $85 \%$ of women, $95 \%$ of men). In this study, current drinkers were defined as individuals who had consumed an alcoholic beverage in the past year. Results showed a similar percentage of weekly drinkers, or individuals who drank at least two alcoholic beverages a week for Japan (30\% of women, $60 \%$ of men) and the U.S. (30\% of women, $55 \%$ of men). Finally, similar results were found for the percentage of heavy drinkers among 18-34 year olds; although heavy alcohol use among women was slightly higher in Japan. Heavy drinkers were defined as individuals who had more than four alcoholic beverages in one sitting. In a separate study of drinking and social norms, Nagoshi, Nakata, Sasano, and Wood (1994) found that social norms for drinking were stronger among Japanese college students compared to American college students. 
The prevalence of heavy alcohol use among men has been noted in several studies. A recent prospective cohort study of 8,934 Japanese adults found that $81 \%$ of males ages $40-49$ were drinkers, with $44 \%$ of drinkers being moderate-to-heavy drinkers (drinking more than at least 4 ounces of pure alcohol per day). This study found that the proportion of abstainers in the sample was similar to the percentage of abstainers reported in the U.S. and in most European countries, however, the proportion of male heavy drinkers was higher in Japan as was reports of drinkers who consume spirits, compared to beer (Sadakane, Gotoh, Ishikawa, Nakamura, \& Kayaba, 2009). Furthermore, one study found that $25 \%$ of men ages $55-77$ were heavy drinkers (consumed more than 8 ounces of pure alcohol per day; Hirayama, Lee, Binns, Okumura, \& Yamamoto, 2009). A second study found that within the Miyagi prefecture $^{3}$ of Japan, $68 \%$ of drinkers were considered moderate-to-heavy users (Nakaya et al., 2007).

In addition to the high levels of drinking among men, recent research suggests that alcohol use may be on the rise among Japanese women. In national public surveys in Japan, researchers found that $76 \%$ of Japanese men and $19 \%$ of Japanese women reported that they were drinkers in 1968 , compared to $78 \%$ of Japanese men and $43 \%$ of Japanese women in 1988 (Higuchi et al., 2006). With more women entering the work force in Japan, women are at increased risk for developing patterns of heavy drinking, as modernism influences encourage women to drink with their professional colleagues, similar to the behaviors traditionally documented among Japanese business men (Higuchi et al., 2006). These concerns are further supported by the 
increase in prevalence of female individuals with alcohol-related problems, which more than doubled from 1984 to 2003 (Osaki, Matsushita, Shirasaka, Hiro, \& Higuchi, 2005). Moreover, Takano, Nakamura, and Watanabe (1996) found alcohol consumption to be most prevalent among women ages 20-39 (compared to other age groups of women). It is particularly important, then, that studies of Japanese consumption include women.

One reason for the high levels of consumption could be that alcohol consumption is considered a social act in Japan, with the underlying belief that drinking is an interpersonal experience shared with others (Shimizu, 2000). Sharing drinking experiences and getting drunk with friends is common and is a societal norm (Shimizu, 2000, 1990); alcohol use is the social lubricant for Japanese society (Wada, Price, \& Fukui, 1998). Moreover, drinking with colleagues after work, in restaurants and izakayas, which are drinking pubs, is seen as a normative behavior (e.g. Higuchi et al., 2006). Furthermore demonstrating this normative behavior is the high prevalence of male workers (who comprise the traditional workforce) who report alcohol-related problems (Higuchi et al., 2006). Thus, drinking with friends is an example of a socially constructed situation, which Kitayama and Park (2007) posits influences and reinforces the interdependent cultural model.

Despite the alarming increase in alcohol consumption among the Japanese population, legislation surrounding alcohol sales and distribution remains liberal; the National Tax Agency in Japan reports that the number of alcohol retailers continues to steadily increase in Japan (2004). Additionally, there is limited punishment for 
underage drinking; although there is a legal drinking age in Japan (i.e. 20 years old), alcohol is available to minors, and the drinking age is poorly enforced (Higuchi et al., 2006). Moreover, alcohol is readily available in vending machines throughout Japan, with only a limited number of the vending machines verifying legal drinking age (Higuchi et al., 2006).

Environmental factors (e.g., availability of alcohol) are important predictors of consumption, as demonstrated among U.S. samples (e.g. Jones-Webb, Toomey, Miner, Wagenaar, Wolfson, \& Poon, 1997). Indeed, Borsari and Carey (2001) confirmed a structural model where the perceived availability of alcohol had direct and indirect effects on binge-drinking for a college student sample of underage drinkers. Perceived availability was defined as whether or not students had access to alcohol. Further, the presence of alcohol outlets, or locations to buy alcohol, also reflects the social norms for drinking within a certain community (Grube, Gruenewald, \& Chen, 2010). The numerous alcohol outlets in Japan, including many that require no check of legal drinking age, demonstrate a high societal acceptance of drinking. Indeed, societal norms toward drinking among youth and women are changing; it is now more socially acceptable for women and youth to drink (Osaki et al., 2005). Therefore, the fact that alcohol legislation remains liberal in Japan may further enhance the social normative context for drinking.

The normative pressures to consume alcohol with friends, experienced by the Japanese, may be similar to the strong normative pressures to consume alcohol experienced by U.S. college students. Despite the differences between the cultural 
selves for these two populations, strong normative pressures to consume alcohol exist for both groups. Specifically, college students are susceptible to pressures to conform (Sears, 1987), especially in terms of alcohol consumption (Baer, 2002). Researchers have identified a strong normative influence for U.S. college students to interact in environments where alcohol was present and to consume alcohol (Perkins \& Berkowitz, 1986), similar to the social influences Shimizu (1990, 2000) outlined among the Japanese. Moreover, Greek members, specifically fraternity men, report the highest level of drinking for both themselves and for their peers (Perkins, 2002). Membership in Greek organizations can be described as a tight collective, where individual members are often described by characteristics representative of their larger organization (Madson \& Trafimow, 2001), similar to the way individuals within interdependent cultures describe themselves (Markus \& Kitayama, 1991).

Evidence for the strong social norms for drinking is found in research documenting alcohol use among U.S. college students. In the U.S., alcohol consumption is highest among college students; two out of five college students report heavy binge-drinking (O'Malley \& Johnston, 2002). Normative pressures for drinking, availability of alcohol, and alcohol advertisements targeted to this population are significant contributors to alcohol consumption among college students (e.g., Baer, 2002; O’Malley \& Johnston, 2002). As previously discussed, these same influences exist in Japan, but apply to the other subgroups, specifically businessmen and more recently women and youth. Given these similarities, it may be beneficial to think of 
the existing literature on U.S. college student drinking when discussing alcohol use in Japan.

\section{College Student Drinking}

As discussed, in the U.S. heavy drinking patterns have been shown to develop during college (e.g. Baer, 2002). Further, as college students prepare to enter the workforce they will likely develop alcohol consumption patterns similar to those who are already in the workforce (Frone, 1999). Adolescence and young adulthood is considered a critical and formative age, as many developmental trajectories are established during this time period and become increasingly difficult to alter later in life (Frone, 2003). Habits or coping methods that students develop during their college years may carry-over into their adult lives (Institute of Medicine, 1998). Within a population where heavy drinking is seen as normative, students who are developing risky drinking behaviors are at increased risk for future dysfunction once they enter the workforce (Higuchi et al., 2006).

In addition to the lack of research focusing on the characteristics of drinking among the general Japanese population, even less research exists which specifically looks at this at-risk population. Existing research shows that alcohol consumption is increasing among adolescents and young adults. Further, alcohol use among Japanese youth has been documented, which is particularly risky, in terms of the development of alcohol-related problems, as alcohol use early in life can reinforce alcohol use as a coping mechanism (Cooper, 1994). Specifically, in a longitudinal study of Japanese high-school students, researchers found that $50 \%$ of junior high-school students and 
$70 \%$ of senior-high school students reported drinking alcohol on at least one occasion (Uehata, Suzuki, Wada, Yamaguchi, Minowa, \& Oida, 2001). Indeed in a multi-year study of alcohol use among Japanese junior and senior high school-aged students, $40 \%$ of girls and $42 \%$ of boys reported drinking with friends in the past 30 days, and $28 \%$ of girls and $33 \%$ of boys reported drinking alone in the past 30 days (Osaki, Tanihata, Ohida, Kanda, Suzuki, et al., 2008). In the same study, $39 \%$ of girls and $51 \%$ of boys reported drinking greater than 3 drinks on one occasion at least once in the past month, with $12 \%$ of girls and $21 \%$ of boys reporting drinking greater than 6 drinks in one occasion in the past 30 days. Finally, the study found that $20 \%$ of students were reporting alcohol-related problems, such as vomiting or experiencing a black out. These behaviors are indicative of the development of future alcohol-related problems, including dependence.

Further supporting these alarming figures of use among Japanese youth, Yeh, Inose, Kobori, and Change (2001) found that $13.4 \%$ of Japanese college students surveyed reported that they drink alcohol to alleviate stress and emotional distress; students who reported a higher collectivist identity were more likely to drink to cope. Drinking to cope is of particular concern, as drinking to cope behaviors are associated with the development of alcohol related problems in U.S. samples (Carey, 1995; Cooper, 1994; Cox \& Klinger, 1988). For example, Cooper, Russell, Skinner, and Windle (1992) found that individuals who endorsed drinking to cope motives showed an increase in substance abuse problems, compared to individuals who endorsed social drinking motives. The relationship between higher collectivist identity and drinking- 
to-cope behaviors documented by Yeh et al. (2001), supports the argument that increases in negative social interactions may play an important role in the decision to consume alcohol; alcohol consumption may be a way to cope with the experience of these negative social interactions.

\section{Social Context of Drinking}

Alcohol researchers have identified solitary and social drinking as two distinct social contexts for drinking. Specifically, solitary alcohol consumption has been associated with drinking-to-cope behaviors and negative mood-related drinking. It is widely accepted that people drink to regulate affect (Cooper et al., 1995). That is, people drink alcohol to manage negative and positive mood, which can be problematic because individuals who drink to cope with negative mood drink more, drink more often and may be at greater risk for alcohol problems, when compared to individuals who drink for social reasons (Cooper, Russell, \& George, 1988). Highlighting contextual differences in consumption, Mohr et al. (2001) found that individuals drank more in solitary contexts on days with high negative interpersonal exchanges and drank more in social contexts on days with high positive interpersonal exchanges. Whereas drinking-to-cope predicted consumption and alcohol problems, social and emotional enhancement expectancies and positive emotions predicted drinking to enhance, which predicted consumption, but not alcohol problems (Cooper et al., 1995). Furthermore, drinking-to-cope behaviors may be more detrimental because reliance on alcohol to regulate negative mood may weaken other methods of coping and create a dependency on alcohol to regulate negative mood (Cooper et al., 1995). 
In a second study of college students, Mohr et al. (2005) found that students drank more with friends on days that they experienced positive mood, which indicates that positive mood drinking may be more social in nature. Moreover, negative mood was indicative of greater drinking both at home and away from home on days when students spent less time with friends, demonstrating a possible solitary component of negative mood-related drinking. As negative mood-related drinking may comprise solitary drinking, individuals may be at increased risk of developing alcohol-related problems as solitary drinking limits the availability of social cues for drinking behavior, and illuminates one's emotional state (Armeli et al., 2003). Thus identifying drinking in these distinct contexts in important. Given the social influences for drinking among Japanese college students, the social context for drinking may be a stronger antecedent for drinking, compared to solitary contexts.

In summary, there are multiple factors contributing to the high levels of alcohol use among Japanese college students. Specifically, the social influences of drinking may make students feel greater pressure to consume alcohol. Moreover, students may drink with friends in an attempt to make amends for previous negative social events. Drinking with friends may be a way students fit in with the larger social group and seek self-improvement, as predicated in interdependent cultural models. Thus, as with other behaviors, understanding drinking behavior within the larger socio-cultural context is important in delineating pathways to consumption. These antecedents of drinking have not previously been examined within this population, despite the documented increase in consumption and cross-sectional reports of 
drinking-to-cope behaviors. As will be discussed in upcoming chapters of this dissertation proposal, studies of Japanese college student drinking should consider socio-cultural influences, social context influences, within-person motivations for drinking, and possible protective factors for drinking, such as drinking refusal selfefficacy and social self-efficacy. This dissertation will investigate antecedents of alcohol consumption. 


\section{CHAPTER IV}

\section{Alcohol Outcome Expectancy Desirability}

In the last chapter, I discussed the Japanese self and alcohol consumption in Japan. I also discussed the impact negative social events have on the Japanese individual and how the individual may choose to make amends for such negative interactions by engaging in social drinking. The act of engaging with others after experiencing a negative or shameful interaction is relatively common to Japan, compared to the United States. Thus, for the Japanese, when one experiences a negative social event, the expected outcome of increasing ties with other through mutual alcohol consumption may seem more desirable, compared to other more solitary situations. The purpose of this chapter is to explain and review such desirability in the expectancy of the effects of alcohol and discuss desirability of alcohol outcome expectancies as a predictor of subsequent alcohol consumption.

First, I will first provide information of how alcohol expectancies are situated within the larger framework of factors influencing drinking behavior. Then, I will provide a review of alcohol outcome expectancies and their relationship to consumption. Finally, I will focus on the desirability of these expectancies and how the desirability is a proximal predictor of alcohol consumption.

\section{Predictors of Alcohol Consumption}

From a biopsychosocial perspective, the decision to drink is multi-faceted and is the result of multiple pre-dispositions, individual decisions, and contextual influences. Specifically, researchers have focused on biological influences, such as 
specific genes related to vulnerability of organ damage (Luczak et al., 2006) and to the development of alcoholism (National Institute on Alcohol Abuse and Alcoholism, 2007). In addition to biological predispositions for the development of alcoholism and alcohol-related problems, personality has also been researched as an individual difference factor predicting alcohol abuse. For example, some individuals are sensation seekers and are drawn to alcohol for it's social enhancement qualities (DelBoca, Darkes, Goldman, \& Smith, 2002). These biological predispositions and personality characteristics, in conjunction with past drinking experiences, influence alcohol outcome expectancies and drinking motivations (DelBoca, Darkes, Goldman, $\&$ Smith, 2002), as shown in Figure 2. Alcohol outcome expectancies are the conscious and subconscious beliefs about the physical and emotional effects of alcohol consumption (Darkes \& Goldman, 1998; Goldman, Del Boca, \& Darkes, 1999), such as "I feel more sociable when I drink." Similarly, drinking motivations are the beliefs about the incentives or reasons for drinking (Cooper, 1994), such as "drinking will make this party more fun." Depending on the context, specific alcohol outcome expectancies and drinking motivations may become more salient (DelBoca et al., 2002). Indeed, certain cultural and contextual influences, such as alcohol availability, social norms, and cultural rituals, interact with alcohol outcome expectancies and drinking motivations, to predict alcohol consumption (Kuntsche, Knibbe, Gmel, \& Engels, 2006). In conclusion, as seen in Figure 2, alcohol outcome expectancies are developed through biological predispositions, cultural and personality factors, and are drawn upon in certain contexts, making them an important proximal predictor of 
alcohol consumption. Alcohol expectancies and drinking motives have been linked to the development of alcohol-related problems, and increases in drinking frequency and quantity among a variety of samples (e.g. Baer, 2002; Goldman, Del Boca, \& Darkes, 1997; Cooper, 1994; Cox \& Klinger, 1988). Further, these beliefs about the outcomes of alcohol consumption are proximal predictors of consumption (Kuntsche et al., 2006), compared to distal predictors of consumption, such as biological predisposition or personality factors. Within the alcohol consumption literature, differences exist between alcohol outcome expectancies and drinking motivations, although both are considered proximal predictors of alcohol consumption. Despite some theoretical differences between the two theoretical concepts, both include motivational properties that influence drinking. Specifically, as will be discussed in this chapter, alcohol outcome expectancies are the beliefs about what will happen when alcohol is consumed, and influence consumption based on whether the individual desires the expected outcome. The desirability of the expected outcome is very similar to the way drinking motivations have been described. Indeed, DelBoca et al. (2002) described the salience of certain alcohol outcome expectancies in specific contexts as a motivational factor for drinking. The remainder of this chapter will draw on the motivational aspects of the alcohol outcome expectancy and drinking motivations literature.

\section{Alcohol Outcome Expectancies}

Cox and Klinger (1988) describe individuals' drinking behavior as a response to incentive motivation. The term incentive motivation is defined as a force to either pursue positive outcomes or to avoid negative consequences. In terms of alcohol 
consumption, an example of a positive incentive could be to reduce tension by drinking, whereas an example of a negative incentive could be to avoid experiencing a hangover. Further, a person who is pursuing an incentive, or desired outcome, is experiencing a motivational state. Cox and Klinger (1988) characterize this state as the final pathway in alcohol consumption (Figure 1).

An individual's decision to drink is multifaceted, however, at the most proximal level to drinking, an individual makes a decision to drink in response to the desired effects of consumption. Cox and Klinger (1988) outline four possible pathways for this to occur: the expectation that positive affect will be enhanced, the expectation that positive affect will be reduced, the expectation that negative affect will be reduced, and the expectation that negative affect will be increased. If the individual expects the outcomes to be positive, the individual drinks. If the individual expects the outcomes to be negative, the individual abstains. These affective responses are influenced by contextual factors (i.e., whether or not others are present and are drinking), and situational factors, (i.e. whether or not alcohol is available). Thus, whereas the expectancy of what will happen when alcohol is consumed is relatively stable, these motivations for drinking are likely to fluctuate.

Expectancy theory is a motivational theory that has been shown to predict a variety of behaviors. Specifically, expectancy theory is a psychological, motivational theory that posits that individuals are motivated to behave in ways that are consistent with their beliefs regarding the outcome of their behavior (Fishbein \& Ajzen, 1975). Expectancies are a learned relationship between stimuli, responses to those stimuli, 
and outcomes (MacCorquodale \& Meehl, 1953), that is, expectancies can be thought of as a summary of an individual's biologically influenced and learned behavior related to a specific outcome (DelBoca et al., 2002). These expectancies are developed in the presence, or in the absence, of actually performing the behavior and can be conscious or automatic (e.g. Goldman, Del Boca, \& Darkes, 1997). Thus, expectancies can be learned through cultural knowledge. Indeed, expectancy theory is a general underlying theory of behavior and has been applied to a variety of behaviors, including aggression (Perry, Perry, \& Rasmussen, 1986), health behaviors (Fishbein \& Ajzen, 1975), and education (Wigfield, 1994).

Specific to alcohol consumption, alcohol expectancies are stable, knowledgebased cognitive beliefs regarding the psychological, behavioral and physical effects of alcohol (Marlatt \& Rosenhow, 1980). In other words, alcohol expectancies are an individual's beliefs about what will happen when one consumes alcohol. For example, "When I drink, I feel more sexual," or "When I drink, I often say things that I later regret."

A sizable amount of research has investigated how alcohol expectancies relate to alcohol consumption. Alcohol expectancies have been shown to be moderators of the relationships between psychosocial variables and alcohol use. For example, individuals may drink alcohol to reduce social anxiety. Built into this process is the assumption that alcohol decreases social anxiety (Goldman, Del Boca, \& Darkes, 1999). 
Goldman (1994) described alcohol outcome expectancies as one of the strongest predictors of alcohol consumption. Alcohol expectancies predict quantity (Carey, 1995), frequency (Fromme, Stroot, \& Kaplan, 1993), initiation and maintenance of alcohol consumption (Brown, Christiansen, \& Goldman, 1987) for a variety of age groups and drinkers (Goldman, Del Boca, \& Darkes, 1999). Carey (1995) found that for college students, expectancies predicted drinking at a one-month follow-up.

Alcohol expectancies have been characterized as either positive or negative (e.g. Brown, Christiansen, \& Goldman, 1987), that is, effects are either desirable or undesirable (DelBoca et al., 2002). Effects of alcohol, such as social or physical pleasure, sexual enhancement, and relaxation are considered positive alcohol expectancies (e.g., Leigh \& Stacy, 1993), whereas effects such as aggressiveness or loss of control are considered negative alcohol expectancies (e.g. Leigh \& Stacy, 1993). Negative expectancies may be inversely related to alcohol consumption, whereas positive alcohol outcome expectancies have been shown to predict increased consumption (Darkes \& Goldman, 1993; Johnson \& Fromme, 1994), and are more predictive of consumption, compared to negative expectancies (Stacy, Widaman, \& Marlatt, 1990; Rohsenow, 1983); cf. Armeli et al., 2005). Leigh (1989) found that global, positive alcohol outcome expectancies accounted for $10-19 \%$ of the variance in current alcohol use. It should be noted that Armeli et al. (2005) found that, at times, negative expectancies are more desirable than positive expectancies, and thus can still 
predict increases in drinking. This finding will be further discussed in the following section of this chapter.

The greater influence of positive expectancies, over negative expectancies, may be due to the timing of positive effects of drinking. Positive effects of drinking, such as increased social lubrication/decreased social anxiety are typically experienced closer to the actual time of consumption, compared to more negative effects of alcohol, such as a hangover (e.g. Cox \& Klinger, 1988). This immediacy of positive effects likely influences positive alcohol outcome expectancies and therefore is more predictive of alcohol use (Leigh \& Stacy, 1993; Abrams \& Niara, 1987). Indeed, in a study of U.S. college undergraduates, Stacy, Widaman, and Marlatt (1990) found that, when in similar drinking situations, positive alcohol expectancies were more accessible in memory than negative expectancies.

Specific alcohol outcome expectancies are strengthened through direct drinking experience, that is, when expectancies are confirmed through experience, the memory of this expectancy is reinforced (e.g. Campbell \& Oei, 2010). Smith, Goldman, Greenbaum, and Christiansen (1995) posited a feedback loop whereby individuals who had positive alcohol outcome expectancies reported greater levels of drinking, which then reinforced positive outcome expectancies. Studies also show that once outcome expectancies are developed, the individual will act out expectancies, even in the absence of alcohol consumption (i.e., when given a placebo; Marlatt \& Rosenhow 1980). 
One study of adolescents (Miller, Smith, \& Goldman, 1990) found that alcohol outcome expectancies crystallized with age. Thus, with additional confirmation of expectancies, an outcome expectancy becomes increasingly difficult to change. However, the desirability of this outcome may change during the life-course, as environmental contexts and situational demands also change (Sher, Wood, Wood, \& Raskin, 1996). I will return to the concept of desirability in the next section.

In sum, alcohol outcome expectancies are the beliefs about what will happen when an individual consumes alcohol. These beliefs can be learned through personal experience, observation, or cultural influences (Goldman, Del Boca, \& Darkes, 1999). Expectancies are predictive of increased consumption and the development of alcoholrelated problems (Carey, 1995; Fromme, Stroot, \& Kaplan, 1993; Brown, Christiansen, \& Goldman, 1987). Moreover, positive alcohol outcome expectancies, that is, outcome expectancies that are considered enjoyable or desirable, are crucial to understanding drinking behavior (Johnson \& Fromme, 1994; Darkes \& Goldman, 1993). However, the beliefs about what happens when alcohol is consumed does not solely predict alcohol use. Indeed expectancies must also be considered in terms of the desirability of expected outcomes. In other words, the expected outcome must be a desirable result in order for an individual to choose to consume alcohol (Armeli et al., 2005).

\section{Alcohol Outcome Expectancy Desirability}

In accordance with other measures of attitudes, an attitude is the combined belief about the outcome and the desirability of the outcome that predicts behavior 
(e.g. Fishbein \& Ajzen, 1975). Whereas alcohol outcome expectancies can be thought of as "if-then" contingencies that reflect learned beliefs about alcohol, a second component of alcohol outcome expectancies, whether or not the outcome is desirable, also exists (e.g. Leigh, 1989). This is the individual's perceived belief about the desirability of the specific outcome expectancy. For example, is the social lubrication provided by alcohol consumption desirable? Is the reduced tension felt after alcohol is consumed desirable? Although expectancies exist, they may not fuel consumption unless the specific outcome is desirable. It is not just the knowledge of the expected outcome, but whether or not the individual wants to experience the expected outcome; it is the desirability of the expected outcome that motivates an individual to drink (Cox \& Klinger, 1988).

In terms of alcohol expectancies, declines in desirabilities are correlated with decreases in drinking across time (Sher et al., 1996). Similarly, the desirability of such outcomes is likely to vary, depending on circumstances such as individual mood and drinking context. Furthermore, there may be environmental or affective cues that activate an outcome expectancy. In fact, in the only study examining daily fluctuations in the desirability of specific alcohol outcome expectancies, Armeli et al. (2005) found daily fluctuations in the desirability of alcohol outcome expectancies; indeed, there were greater fluctuations within-person than there were between-person, indicating that individual ratings of desirability varied by day. Specifically, alcohol outcome expectancy desirabilities were higher on days with higher positive affect (compared to lower positive affect), higher negative affect (compared to days with lower negative 
affect), and on days with a higher number of negative social events (compared to days with lower negative social events). Finally, Armeli et al. (2005) found that desirabilities significantly and positively predicted evening drinking.

Whereas alcohol outcome expectancies and drinking motives have been distinguished as separate constructs, the desirability of expected outcomes may be similar to drinking motives. Alcohol outcome expectancies are inherent in both the desirability of anticipated outcomes and motives for drinking. Both desirability of anticipated outcomes and drinking motives are based on the appealing effects of consumption. Alcohol expectancies influence drinking motives by informing what the outcomes will be; alcohol expectancies are inherent in drinking motives. Specifically, Cox and Klinger (1988) posited that motivated drinking is in response to expectations about the reactions to alcohol consumption, whether the reactions are biological (i.e. due to alcohol's chemical affects) or affective in nature.

Kuntsche et al. (2005) found that drinking motives, such as "I like the feeling of drunkenness" or "I drink to get high," mediated the relationship between alcohol outcome expectancies and drinking. Defined in this way, drinking motives are similar to the desirability of alcohol outcome expectancies that Armeli et al. (2005) used in their study (e.g. "A pleasant feeling from drinking alcohol with friends."). In conjunction with the Armeli et al. (2005) study, these studies demonstrate the importance of the desirability of expected outcomes in predicting drinking.

In summary, alcohol outcome expectancies are an important predictor of alcohol consumption. Their predictability is aided by measuring the desirability of 
such outcomes. Desirability of outcomes fluctuates as a result of environmental and affective states. Therefore, as discussed in Chapter 6, measuring alcohol outcome expectancy desirability at a daily level is an appropriate way to capture changes in desirability that serve as more proximal predictors of drinking. Specifically of interest to this dissertation is the finding that alcohol outcome expectancy desirability increases on days with greater negative social events compared to days with fewer negative social events. Desirability of alcohol outcome expectancies is likely to fluctuate; therefore an interview protocol that captures such variations in desirability would be ideal. Daily process methodology includes multiple interviews during the course of the study and is an advantageous method for tracking such fluctuations. In the next chapter, I will present daily process methodology and discuss its usefulness in capturing these types of fluctuations. 


\section{CHAPTER V}

\section{Daily Process Methodology}

I have just presented two possible proximal predictors of alcohol consumption among Japanese college students: daily negative social events and alcohol outcome expectancy desirability. Both of these predictors are likely to have day-to-day fluctuations in occurrences. The purpose of this chapter is to describe daily process methodology, which is an ecological form of measurement that captures data at multiple time points for each participant, and is a suitable form of measurement for these types of variables. First, I will outline field observational methods, paying special attention to daily process methodology in particular. After defining daily process methodology, I will describe the benefits of a daily process approach.

\section{Field Methods}

Beginning in the 1980s, a major shift in social psychology occurred (Jones, 1998). Some researchers were becoming increasingly concerned with the field's reliance on data collected in lab experiments, using college sophomores as participants (e.g., Sears, 1980). Researchers began to call for a change in research methodologies and changes in research programs. Field research, including non-obtrusive observational methods were encouraged (e.g., Wortman, Abbey, Holland, Silver, \& Janoff-Bulman, 1980).

The opportunity to capture naturally occurring events during a specific period of time is one of the facets of field methods. This form of data collection is enriched because it has ecological realism and includes a variety of methods, such as 
observations, survey research, and daily process methodology. These reports allow for a more detailed, accurate, multifaceted picture of social behavior as it occurs in a natural context (Bolger, Davis, \& Rafaeli, 2003; Reis \& Gable, 2000).

\section{Daily Process Methodology}

Daily process methodology is characterized as the collection of data on a recurring basis (e.g., once per day, once per week) using various types of recording schedules, ranging from certain time interval, to event contingent intervals (e.g., Reis \& Gable, 2000). This data is collected in a variety of formats, including internet surveys (e.g., Mohr et al., 2005), hand-held computers (e.g., Mohr, et al., 2003), telephone surveys (e.g., Holtzman \& DeLongis, 2007), and pen and paper surveys that are subsequently mailed back to the researcher (e.g., Stone \& Shiffman, 1994). The cornerstone of daily process methodology is multiple repeated measurements from the same individual that occur close in time to the occurrence of the event of interest. This type of measurement is similar to following a person throughout their daily life and recording their behavior, only less obtrusive (Reis \& Gable, 2000; Reis, 1994).

Using daily process methodology more accurately captures certain behaviors and events, compared to one-time self-report measures, which is one of the main advantages of daily process methodology over other forms of survey methods. These phenomena are those that are highly subject to retrospective bias, meaning making, salience, and recency. Such phenomena include minor events, social interactions, affect, and drinking behavior (Reis \& Gable, 2000). For example, retrospective reports of mood are often contaminated by problems such as exaggerations of the magnitude 
of the mood (Thomas \& Diener, 1990) and variability in mood experiences (Robinson \& Clore, 2002). Similarly, daily events and interactions are often easily forgotten over time, but do cause detriments to well-being (Reis \& Gable, 2003; Bolger, DeLongis, Kessler, \& Schilling, 1989; DeLongis, Folkman, \& Lazarus, 1988). Finally, retrospective reports of alcohol consumption may be incorrect, in that people cannot recall drinking contingencies (Carney et al., 1998).

In addition to capturing more accurate reports of social interactions and alcohol consumption, daily process methods allow for researchers to test hypotheses regarding the relationship between social interactions and drinking. In order to accurately measure drinking in response to social interactions, drinking and social interactions must be captured as they occur (Carney et al., 1998) which is difficult to do using onetime self-report measures. Indeed, according to models of stress and coping, as will be discussed in the following chapter; a process exists whereby a stressor occurs and a coping method is chosen to alleviate the stressor (Lazarus \& Folkman, 1988). In order to accurately capture this process as it unfolds, contemporaneous reports of behavior are a necessity. Daily process methods are considered contemporaneous reports of behavior, and are particularly important when investigating drinking in response to daily interactions and events (Tennen \& Affleck, 1996; Tennen, Affleck, Armeli, \& Carney, 2000). In conjunction with multi-level modeling, daily process data can be used to create temporal associations, capture intra- and inter- individual changes, and create individual trajectories (e.g., Affleck, Zautra, Tennen, \& Armeli, 1999; Tennen, Suls, \& Affleck, 1991). 
Daily process methodology is commonly used to obtain information regarding the prevalence and characteristics of phenomena (Reis \& Gable, 2000). In other words, how often does a specific event happen? Daily process methodology can also be used to test hypotheses regarding within-person or between-person processes, or to test hypothesis with ecological realism. That is, daily process methodology enhances the ecological validity of studies. Ecological validity is the extent to which the research methodology, process and data collection mirror a real-life situation where the variable(s) of interest naturally occur (Shadish, Cook, \& Campbell, 2002; Brewer, 2000). Finally, these techniques can be used to discover how events unfold (Bolger, Davis, \& Rafaeli, 2003; Reis \& Gable, 2000). Daily process methods allow the researcher to investigate everyday experience. These experiences may differ from experiences found within the laboratory, or captured from self-reports remotely following an event (i.e., questionnaires that ask participants to think about their behavior over the past month) for several reasons.

First, in the laboratory, participants are aware that they are being studied; this may influence the participants to act in a certain way. In daily process studies, the diary assessment becomes assimilated into the participant's life and allows for a more accurate representation of behavior (e.g., Stone, Shiffman, \& DeVries, 1999; Stone \& Shiffman, 1994). Second, participants may have difficulties remembering events exactly how they occurred in the past. Human memory is subject to retrospective bias and meaning making. Specifically, as time passes following an event, individuals are able to understand events in a larger contextual framework, can make amends, or can 
justify behavior (e.g., Bolger, Davis, \& Rafaeli, 2003; Reis \& Gable, 2000; Stone, Shiffman, \& DeVries, 1999). Individuals are likely to recall the event better if it happened recently (recency effect) or caused greater disruption in their daily life (salience). Individuals are also likely to forget about minor events, such as negative social interactions with strangers. However, these events, even though minor, if stressful can decrease well-being over time (Reis \& Gable, 2003; Bolger et al., 1989; DeLongis, Folkman, \& Lazarus, 1988). Finally, multiple reports of similar behaviors/events allows for fluctuations in these behaviors/events to be captured. Fishbein and Ajzen (1975) suggest that one of the best ways to accurately capture behavior is to evaluate a behavior at multiple time points; a criterion satisfied by daily process methodology. Further, Shiffman (2000) demonstrated that aggregates of dairyreported behavior had a closer fit to actual behavior (as reported via signal contingent daily reports of smoking behavior), compared to retrospective reports, thus demonstrating that daily diary data have greater validity and reliability, due to decreases in measurement error (Bolger, Davis, \& Rafaeli, 2003).

\section{Types of Daily Process Protocols}

In general, there are three types of ecological momentary assessments: signalcontingent, event-contingent, and interval-contingent (Reis \& Gable, 2000; Wheeler \& Reis, 1991). In signal contingent methods, participants are prompted to complete a survey by some sort of an alarm. This signal is commonly in the form of a pager or an alarm that is carried with the participant. The signal can be set to randomly occur. When signaled, participants report their current behavior. Signal contingent protocols 
are preferable when determining the prevalence of events in daily life. In an eventcontingent protocol, participants complete a diary survey after a specific event occurs (as specified by the researcher). The event-contingent protocol, similar to the signalcontingent protocol, is useful in determining the prevalence of events in daily life.

The third type of daily experience protocols is interval-contingent recording. In this protocol, participants report their experiences at regularly, predetermined intervals. Interval-contingent protocols have less participant burden, compared to event-contingent and signal-contingent protocols, as the participant is aware of the time that they will complete a survey. This allows for the participant to plan ahead for survey completion. Participants also do not have to carry the recording device with them during their regular day (thus allowing for computer-based surveys). The disadvantage of interval-contingent designs is that the report may be somewhat removed from the event, although this report is still preferred over one-time selfreports that ask participants to recall events over a larger period of time. Indeed Perrine, Mundt, Searles, and Lester (1995) found a significant correlation between biological and retrospective reports of alcohol consumption during a 24-hour period. Thus, an interval contingent protocol is acceptable, as long as data is collected within 24-hours of the event.

The current study will use data that were collected via an interval-contingent protocol in order to reduce participant burden and to collect daily reports of alcohol consumption, negative social events, and alcohol outcome expectancy desirability. This type of protocol was chosen over an event-contingent protocol because the 
interval-contingent protocol does not require the participant to carry the data collection device with them at all times, and allows for participants to plan in advance to answer the daily survey questions. For this same reason, a signal-contingent protocol was not chosen. Additionally, event- and signal-contingent protocols are often used to establish the frequency of events, which is not a purpose of the current study. The purpose of the current study is to identify a temporal relationship between events; thus, an interval-contingent protocol is preferred over signal- and event-contingent protocols.

Regardless of the specific type of protocol that is used to collect data (i.e., event-contingent, interval-contingent, signal-contingent), there are important considerations the researcher must make in order to ensure the daily process data are accurately captured. Specifically, it is imperative to ensure that participants complete the surveys at the appropriate time and do not change their answers (Green, Rafaeli, Bolger, Shrout, \& Reis, 2006). If the researcher cannot ensure that participants did not "backfill" their surveys, or complete their diaries when appropriate, daily process data have no benefits over traditional self-report data (Tennen, Affleck, Coyne, Larsen, \& DeLongis, 2006). One way to ensure that diaries are completed at the appropriate time and that participants do not change their answers is to have participants complete their diaries using a web survey, which time-stamps the survey and restricts access to the survey.

Daily process methodology allows researchers to capture both daily interactions and events and drinking behavior on a daily basis, thus enhancing 
measurement, as well as allowing researchers to establish the temporal relationship between these events and consumption. In conclusion, using daily process methods, researchers are able to capture events in the sequence they occur, capture fluctuating processes closer to "real-time," and minimize recall error (Tennen \& Affleck, 1996). Therefore, daily process methodology is an appropriate methodological choice when studying fluctuations in the desirability of alcohol outcome expectancies, the occurrence of social events and interactions, and daily alcohol use.

Now that I have discussed the possible proximal predictors of alcohol consumption among Japanese college students and presented daily process methodology as an appropriate tool to measure these predictors, I will discuss possible protective factors for drinking. Specifically, researchers have documented that certain types of self-efficacy, or one's belief about their ability, reduce the amount of alcohol an individual consumes. The next chapter will discuss two types of self-efficacy, drinking refusal self-efficacy and social self-efficacy, which may be resources specific to reducing alcohol consumption among this population. That is, these types of selfefficacy may act as moderators in the relationship between negative social events and drinking. 


\section{CHAPTER VI}

\section{Self-Efficacy}

I have previously discussed daily negative social events and alcohol outcome expectancy desirability as proximal predictors of alcohol consumption. I have also discussed an appropriate methodology for studying these predictors, given the frequent fluctuations found for these variables. The purpose of this chapter is to discuss selfefficacy as a possible moderator of the previously mentioned relationships.

Self-efficacy is an individual's beliefs about their ability to perform a certain behavior, in this case, to abstain from, or limit alcohol consumption. That is, selfefficacy may be a potential protective factor for drinking. This chapter will focus on self-efficacy. First, I will define and discuss general self-efficacy. I will discuss the benefits of including self-efficacy in the prediction of behavior and how self-efficacy fits into a larger model of health behavior. I will also present the difficulties in measuring self-efficacy. Then, I will present two task-specific types of self-efficacy that are especially relevant to this dissertation: drinking refusal self-efficacy and social self-efficacy.

Self-efficacy

Self-efficacy is the belief that one has the ability to act in a certain way or achieve a certain goal (Bandura, 2004). Self-efficacy influences the way people interact with others and their environment. It also influences perceptions of the self (Bandura, 1992). Self-efficacy influences an individual's health behaviors through cognitions about their ability to either perform a behavior or not perform a behavior, 
through their behavioral choice, through the amount of effort enacted toward a behavior, and in perseverance in response to adversity (e.g., Bandura, 1977, 1997, 2004).

Individuals with low perceived self-efficacy expect negative, or poor, outcomes from their behavior, whereas individuals who have high perceived selfefficacy expect positive outcomes from their behavior (Bandura, 2004). Research has shown that efficacy beliefs predict a variety of behaviors, including work performance, academic achievement, athletic performance, and health functioning (Bandura \& Locke, 2003).

Central to the construct of self-efficacy is the assumption that people make attempts to avoid engaging in tasks where efficacy is low, and seek out tasks where efficacy is high (Bandura, 1986). Moreover, multiple researchers have posited that individuals perform best and have the greatest well-being when efficacy judgments are slightly higher than actual ability (e.g., Csikszentmihalyi, 1997; Bandura, 1986). Selfefficacy differs from actual ability, as it is an individual's perception of their ability, not their actual ability. This creates an opportunity for misperceptions in ability and can be maladaptive (Bandura, 1986). For example, as is commonly seen among firstyear graduate students, students enter the new environment with high academic selfefficacy due to their exceptional performance during their undergraduate careers. Upon entering the new graduate school context, students are met with greater challenges, and if self-efficacy is too high, and effort does not increase, the student will likely fail, causing detriments to her/his well-being. Moreover, actual 
performance can increase (or decrease) self-efficacy over time, as a result of accomplishments (or failures). However, individuals with high self-efficacy usually attribute failure to extenuating circumstances, compared to individuals with lower selfefficacy who internalize the failure (e.g., Bandura, 1986). As a result of these fluctuations in experiences, and evaluations, self-efficacy is not static (Salovey, Rothman, \& Rodin, 1998).

A variety of health behavior models posit that self-efficacy has direct and indirect effects on behavior. For example, in the theory of planned behavior (TPB; Ajzen, 1991), attitudes, norms and self-efficacy influence behavior indirectly through intention, while self-efficacy also has a direct effect on behavior (whereas attitudes and norms do not). Similarly, the value-belief-norm health model also includes selfefficacy as a direct predictor of behavior (Stern, Dietz, Abel, Guagnano, \& Kalof, 1999). Thus, self-efficacy is an important component of behavior. Self-efficacy influences health as fluctuations in self-efficacy also influence changes in affect, motivation, and action (Bandura, 1992). Health models posit that self-efficacy can be increased by providing individuals with the knowledge and skill-set to perform a behavior, and with mastery experiences, or opportunities to practice the behavior. For example, self-efficacy to use a condom was increased in a study where women role played talking with their sexual partner about using a condom, were involved in discussions surrounding condom use, and learned ways to talk about condom use (Bryan, Aiken, \& West, 1996). The learning component of self-efficacy makes it an important factor in prevention and intervention efforts (Bandura, 1992, 1986). 
Self-efficacy is an integral component of the coping process (e.g., Bandura, 1986). Lazarus and Folkman (1984) proposed the transactional model of stress and coping (Figure 3 represented a customized adaptation of the model to fit alcohol consumption). According to this model, when an individual experiences a potential threat to well-being (i.e., a stressor), the individual seeks to restore balance (Lazarus \& Cohen, 1977). In other words, some events cause chaos in our lives. In order to reduce this distress, individuals take action to mitigate the stressful event. The transactional model is a framework for understanding and evaluating the process by which the individual addresses the stressor. According to the model, the individual interacts with the environment in order to address and alleviate the stressor. When the individual is first faced with a stressor, a primary appraisal is made. This appraisal is the individual's judgment regarding the significance of the event and whether the event is controllable, challenging or irrelevant. After assessing the event, a second appraisal is made. The second appraisal is an evaluation of the individual's resources to deal with the event. Secondary appraisals are based on the individual's perception of how they can control or alleviate the event.

Following the transactional model of coping (Lazarus \& Folkman, 1984), when confronted with a stressor, a primary appraisal is made. If the stressor is appraised as a threat, a secondary appraisal is made of how to combat the stressor. Self-efficacy affects the secondary appraisal process (Figure 3; Jerusalem \& Schwarzer, 1992; O'Leary, 1992). According to Bandura (1992, 1986), perceived selfefficacy influences the individual's perceived ability to exercise control over the 
stressor. If an individual has confidence in their efficacy to address the event (i.e., selfefficacy is high), the secondary appraisal will involve a positive evaluation of the resources available to combat the stressor, thus presenting a challenge for the individual. If an individual doubts their efficacy (i.e., self-efficacy is low), the individual will appraise the stressor as a threat. If an individual feels they cannot combat the stressor, a detriment to well-being is experienced.

As a result, self-efficacy plays a central role in arousal. If an individual perceives a stressor as a threat and has low self-efficacy for addressing the stressor, anxiety and physiological responses increase (i.e., increases in heart rate and catecholamine release), whereas high self-efficacy actually reduces the negative stress response (e.g., Bandura, 1992, 1986; O’Leary, 1992; Salovey, Rothman, \& Rodin, 1998). For example, individuals who believe they have the skills to complete a task or overcome a stressor, experience less anxiety and smaller increases in heart rate and blood pressure. Individuals with low self-efficacy, compared to individuals with high self-efficacy, also report lower challenge and higher threat appraisals (Jerusalem \& Schwarzer, 1992). Low self-efficacy to combat stressors has also been linked to weakened immune functioning (Bandura, 1992; Coe \& Levine, 1986). Finally, individuals with low self-efficacy experience greater loss in response to failures, compared to individuals with higher self-efficacy (Jerusalem \& Schwarzer, 1992).

According to Bandura's $(1986,1977)$ original conception of self-efficacy, there are two types of expectancies related to self-efficacy: efficacy expectancy, or the individual's beliefs regarding their ability to perform a behavior, and outcome 
expectancy, which is the belief about consequences regarding the activity (similar to alcohol outcome expectancies; Bandura, 1986, 1977). Bandura discriminated between general self-efficacy, which is a broad belief about self-efficacy, and task-specific selfefficacy, which is self-efficacy regarding a particular behavior.

Bandura (1992) states that task-specific self-efficacy is a more appropriate predictor of specific behavior and encourages the use of task-specific measures of selfefficacy in the prediction of behavior. In other words, an individual may have high academic self-efficacy (a task-specific type of self-efficacy), which increases performance in familiar academic contexts, but high academic self-efficacy will likely not predict performance in a medical emergency. Oei, Hasking, and Phillips (2007) confirmed this distinction in a study investigating drinking refusal self-efficacy (a specific type of self-efficacy related to the perceived ability to decline alcohol) and drinking. Participants mailed in survey responses regarding their drinking refusal selfefficacy and drinking outcomes. The cross-sectional results showed that drinking refusal self-efficacy was a stronger predictor of quantity and frequency of consumption, compared to general self-efficacy. Thus, task-specific types of selfefficacy may be beneficial to the prediction of behavior. I will discuss the drinking and social relationship task-specific types of self-efficacy later in this chapter.

Finally, it is important to establish a temporal sequence of self-efficacy predicting behavior. In order to predict a causal relationship between self-efficacy and behavior, self-efficacy beliefs must occur (and be measured) prior to behavior (Judd \& Kenny, 1981). Specifically, in order to influence behavior, self-efficacy must occur 
prior to the behavior. Furthermore, Salovey et al. (1998) suggest that self-efficacy should be specific to the measured behavior. For example, if the researcher is interested in studying academic achievement, a measure of general self-efficacy may be too broad to capture actual beliefs regarding the individual's self-efficacy toward academic testing. Following this suggestion of specificity, drinking refusal selfefficacy, which is specifically related to alcohol consumption, and social self-efficacy, a specific measure of one's perceived beliefs about their ability to create and maintain relationships, will be used in this dissertation in order to predict drinking and social behaviors.

\section{Drinking Refusal Self-efficacy}

Drinking refusal self-efficacy (DRSE) is a task-specific type of self-efficacy. It is an individual's confidence or beliefs about her/his self-control behavior in certain drinking contexts (Baldwin, Oei, \& Young, 1993; Lee \& Oei, 1993; Oei \& Burrow, 2000; Young, Connor, Ricciardelli, \& Saunders, 2006). Specifically, DRSE is an individual's belief about her/his ability to resist or refuse alcohol (Baldwin, Oei, \& Young, 1991). Drinking refusal self-efficacy is inversely related to consumption, as it represents an individual's perceived ability to decline drinks (Lee \& Oei, 1993). Individuals who report a greater inability to either refuse drinks in situations where drinking occurs, or who are unable to limit the number of drinks they consume are described as having low self-efficacy for drinking refusal (Oei, Hasking, \& Young, 2005). There are three dimensions of self-efficacy for drinking refusal: social pressure, emotional relief, opportunistic drinking (Lee \& Oei, 1993). Social pressure is defined 
as drinking because one wants to adhere to the social norm. Drinking for emotional relief is characterized as drinking to alleviate an aversive mood (Lee \& Oei, 1993). Finally, opportunistic drinking is defined as drinking in certain situations, such as drinking at lunch, or drinking on the way home from school. DRSE is predictive of drinking levels, frequency of drinking episodes, and can distinguish problem drinkers from non-problem drinkers (Oei \& Young, 1993; Young et al., 2006; Lee \& Oei, 1993). Moreover, DRSE has been found to be a better predictor of alcohol consumption, compared to general self-efficacy (Oei \& Sweeney, 1993).

In a survey of college undergraduates, Young et al. (2006) found that DRSE predicted $50 \%$ of the variance in alcohol dependence, frequency of drinking, and quantity of alcohol consumed on each occasion. Similarly, in a sample of college undergraduates, Oei and Morawska (2004) found that DRSE predicted both quantity and frequency of drinking. Thus, DRSE is important when predicting the number of drinks an individual will consume in certain contexts, as well as whether or not the individual chooses to drink. Additional studies show that when given the opportunity to drink, individuals with low DRSE choose to consume a greater number of drinks, compared to individuals with higher DRSE (Hasking \& Oei, 2002; Lee \& Oei, 1993).

Thus, drinking refusal self-efficacy is an important contributor to the decision to drink and to keep drinking. Further, compared to general self-efficacy, drinking refusal self-efficacy is more specific to alcohol consumption and therefore is a better predictor of consumption. 


\section{Social Self-Efficacy}

A second type of task-specific self-efficacy is social self-efficacy. Social selfefficacy is an individual's beliefs about their ability to engage in social tasks that are central to the initiation and maintenance of social relationships (Smith \& Betz, 2000). Social self-efficacy includes beliefs of skills such as participation in group activities, friendly behaviors, ability to initiate social contact, and the ability to appropriately respond to relationship difficulties (Bandura, 1977; Gecas, 1989). People with high social self-efficacy have greater confidence about their ability to address interpersonal difficulties and also utilize more effective problem-solving strategies (Bilgin \& Akkapulu, 2007). Social self-efficacy has important outcomes related to one's social relationships (O'Leary, 1992). Specifically, social self-efficacy is reinforced by positive (or negative) evaluations from one's social group. These evaluations and perceptions of social self-efficacy may affect the structure of an individual's social network (in terms of opportunities for social support), as well as the functionality of members of the network. These interpersonal relationship factors have repeatedly been shown to predict well-being and physical health-related outcomes (e.g., Cohen \& Wills, 1985; House, Umberson, \& Landis, 1988; Stroebe \& Stroebe, 1996). Thus, social self-efficacy is an important contributor to interpersonal relationships and, as a result of this influence, also to health and well-being.

A number of studies have investigated social self-efficacy among children, adolescents, and young adults. In a study of depression in children, Bandura, Pastorelli, Barbaranelli, and Caprara (1999) found that social self-efficacy had direct 
and indirect effects on depression. Further, in a sample of high school students, Bandura (1997) found a positive relationship between social self-efficacy and emotional well-being. Similarly, Gresham, Evans, and Elliott (1988) found social selfefficacy predicted sociometric status in $3^{\text {rd }}-5^{\text {th }}$ graders. Finally, Vieno, Santinello, Pastore, and Perkins (2007) found that social self-efficacy predicted high life satisfaction at a five-year follow-up for a sample of junior high school students.

In a study of Japanese high school students, Matsushima and Shiomi (2003) found that social self-efficacy was negatively correlated with interpersonal stress and was positively correlated with interpersonal stress coping (which includes measurement of positive coping, expectation of support, and cognitive coping). Further, individuals who were characterized as having high social self-efficacy and either low or high coping responses reported lower levels of interpersonal conflict, feelings of interpersonal inferiority, and interpersonal dislocation, compared to individuals who where characterized as low social self-efficacy.

Social self-efficacy has also been investigated as an important predictor of life satisfaction, well-being, loneliness, and symptoms of depression among traditional college students. This developmental time period may be particularly sensitive to social self-efficacy, as it is a time period where many students are exposed to new social networks and are leaving a familiar environment (e.g., Arnett, 2000). A negative relationship between social self-efficacy and depression has been well-documented among college students (Smith \& Betz, 2002; Saltzman \& Holahan, 2002; Hermann \& Betz, 2006). Social self-efficacy has also been found to have a negative relationship 
with loneliness (Hermann \& Betz, 2006), including a meditational relationship between attachment style and reports of loneliness at a six-month follow-up (Wie, Russell, \& Zakalik, 2005). Finally, DeWitz and Walsh (2002) found that social selfefficacy was significantly correlated with satisfaction with college among a sample of college students.

Social self-efficacy has also been investigated among a variety of worker samples. In a study of telephone hotline workers, Xie (2007) found that social selfefficacy moderated the relationship between mid-shift stressors and end of shift reports of participant stress, such that stressors measured midway through a worker's shift had an ameliorating effect for individuals with high social self-efficacy, compared to those with low social self-efficacy. In a study of management work teams, DeSivilya and Eizen (2005) found that individuals with high levels of social self-efficacy reported a desire to engage with coworkers, who were members of a management team, in response to interpersonal stress. This type of engagement was defined as an engagement-constructive mode of conflict resolution and was related to a greater functioning of the group. Thus, high social self-efficacy workers were less influenced by stressors, and chose more constructive conflict resolution strategies, compared to individuals with lower social self-efficacy.

Although social self-efficacy has not been studied directly in relationship to alcohol consumption, it is a significant predictor of interpersonal relationships and well-being. As I discussed in Chapter II, a relationship between social events and interactions, and drinking exists. Social self-efficacy may interact with this 
relationship and provide a protective buffer against the deleterious effects of negative social events for individuals with high social self-efficacy. In contrast, social selfefficacy may also influence consumption in social settings, where drinking is considered the normative behavior. These relationships will be investigated in the proposed study. 


\section{CHAPTER VII}

\section{Development of Research Questions and Hypotheses}

In the preceding sections, I have discussed important predictors of alcohol consumption particularly among Japanese college students (see Figure 1). I have identified negative social interactions as significant events for the Japanese and drinking in response to such events as an important cultural influence. I have also discussed the importance of desirability of alcohol outcome expectancies as a withinperson predictor of consumption. Finally, I have discussed drinking refusal selfefficacy and social self-efficacy as possible between-person moderators of alcohol consumption. I have discussed each of these constructs as predictors of alcohol consumption, which have previously been studied as separate constructs. Now, I turn to the current study, which proposes investigating how these constructs influence each other and work together to predict alcohol consumption.

The current study is the first study of its kind to investigate the interrelationships between daily negative social interactions, the desirability of alcohol outcome expectancies and drinking among a Japanese college student sample. This study will be the first to identify within-person variation in drinking among this population. These data will provide a more detailed description of alcohol use among a Japanese college student sample; previous studies have relied on one-time selfreports of alcohol use during the past 30-days to one year. This will also be the first study to investigate fluctuations in the desirability of alcohol outcome expectancies among a Japanese sample. Further, the current study will add to the self-efficacy 
literature by investigating drinking refusal self-efficacy and social self-efficacy as between-person moderators in the relationship between negative social interactions and alcohol consumption.

The Proposed Study

Given the strong social influences on behavior, and the strong desire to make amends following negative social interactions that occurs within Japanese culture, the current study, using data collected via daily process methodology, will investigate the relationships between daily negative social events, daily alcohol outcome expectancy desirability, self-efficacy and drinking with others. Specifically, using data collected via daily process methodology, this dissertation will examine whether Japanese students drink more with others on days with greater negative social events relative to days with fewer negative social events (see Figure 1).

Second, this dissertation will investigate whether daily negative social events predict daily alcohol outcome expectancy desirability, such that expectancies related to relationship maintenance and tension reduction are more desirable on days characterized by negative social events, relative to days with fewer negative social events. Third, this dissertation will examine the relationships between drinking refusal self-efficacy and social self-efficacy, and drinking. In addition to examining the main effects of these types of self-efficacy on drinking, this dissertation will also investigate drinking refusal self-efficacy and social self-efficacy as cross-level moderators of negative social events and drinking with others. 
As I described in earlier sections of this dissertation, this study will use data previously collected from a sample of Japanese college students studying in the U.S. and in Japan. The data were originally collected as part of a larger project on crosscultural adjustment and health. During the 30-day study, students completed daily web-based diaries of events and health outcomes, such as drinking.

\section{Hypotheses and Research Questions}

As discussed in Chapter 2, negative social interactions are the most harmful of daily stressors (Bolger et al., 1989) in terms of detriments to well-being. Furthermore, negative social interactions may be even more harmful for individuals in collectivist cultures, where an emphasis is placed on harmony within interpersonal relationships (Markus \& Kitayama, 1991). However, in comparison to more independent cultures, where individuals may more commonly seek out isolation in response to negative interactions, in interdependent cultures, such as Japan, individuals may more commonly seek out network members in an effort to repair the relationship or to save face (Mascolo, Fischer, \& Li, 2003). As discussed in Chapter 2, sharing alcoholic drinks with friends is considered a positive interpersonal experience within Japanese culture (Wada, Price, \& Fukui, 1998).

Moreover, drinking is a socially prescribed act in Japan (Shimizu, 1990). Consuming alcohol with friends is a way to build and strengthen relationships. Given the socially engaging response to negative interactions, and the strong social norms for drinking with friends, it is reasonable to assume that on days with greater negative social events, compared to days with fewer negative social events, Japanese college 
students will drink more with others, as demonstrated by the proposed theoretical model in Figure 1. On the basis of these assertions, I propose the following:

Hypothesis 1: Students will drink more with others on days characterized by greater negative social events compared to days with fewer negative social events.

According to existing research, alcohol outcome expectancies or the beliefs about the effects of alcohol consumption predict alcohol consumption (Goldman, Del Boca, \& Darkes, 1999). For example, the belief that alcohol will decrease tension influences an individual's decision to consume alcohol in order to relieve tension. Specifically for Japanese college students, the belief that consuming alcohol will increase closeness between oneself and one's friends is an important component of drinking behavior. For example, Wada, Price, and Fukui (1998) found that adolescents and young adults mirrored their drinking behavior to match older Japanese adults drinking behavior, which focused on drinking as an interpersonal experience shared with friends. However, in order for the expectancy to predict consumption, the individual must desire the expected outcome. A second component of expectancies is the desirability of such expectancies (Armeli et al., 2005). There are fluctuations in the desire to elicit a certain outcome. That is, on some days, certain outcomes related to alcohol consumption may be more desirable. As previously mentioned, negative interpersonal events are extremely stressful, especially to individuals within an interdependent culture, where an emphasis is placed on maintaining social relationships and social harmony. Following the Japanese cultural model, I predict it is 
more likely that an individual will seek out others in order to make amends for the negative event. Thus, alcohol outcome expectancies related to tension reduction and enhancing social relationships are likely to be more desirable following the experience of negative social interactions. Hypothesis 2 and 3 are proposed as follows:

Hypothesis 2: The desirability of alcohol outcome expectancies related to relationship building and tension reduction will be higher on days with greater negative social events, compared to days with fewer negative social events. Hypothesis 3: Drinking with others will be higher on days when alcohol outcome expectancies related to tension reduction and relationship building are rated as more desirable, compared to days with lower rated desirability. Whereas negative social events may increase drinking, individuals' beliefs about their ability to abstain or limit their drinking in certain contexts may serve as a between-person protective factor. Specifically, existing research demonstrates an inverse relationship between drinking refusal self-efficacy and consumption (Oei, Fergusson, \& Lee, 1998). In other words, the more individuals believe that are able to decline offers of alcoholic beverages in certain situations, the more likely they are to actually decline offers. This relationship will also be investigated for the current sample:

Hypothesis 4: Drinking refusal self-efficacy will significantly and negatively predict drinking with others.

Moreover, drinking refusal self-efficacy may act as a moderator in the relationship between negative social events and drinking. Individuals who have a high 
perceived ability to decline drinks may be less likely to turn to alcohol consumption with friends as a way to make amends after experiencing negative social interactions. Therefore, drinking refusal self-efficacy may serve as a buffer (or a moderator) in the relationship between negative social events and drinking. Thus, Hypothesis 4 is:

Hypothesis 5: Negative social events will have less of an effect on drinking for individuals who have high perceived drinking refusal self-efficacy, compared to those with low drinking refusal self-efficacy.

Finally, a second protective factor may be social self-efficacy. Social selfefficacy is the beliefs about one's ability to engage in social tasks that enhance relationships (Smith \& Betz, 2000). Individuals who have high social self-efficacy perceive themselves to be adept at facilitating group interactions, maintaining and initiating relationships, and appropriately responded to relationships concerns (Gecas, 1989; Bandura, 1977). Thus, individuals with high social self-efficacy may be less affected by negative social interactions; they may have the perception that they have the resources to repair the relationship. Therefore individuals who have high social self-efficacy may feel less pressure to consume alcohol with friends in order to repair relationships. However, the reverse relationship may also be true; given the importance in Japanese culture of drinking with friends, it may be individuals who have higher social self-efficacy that are more willing to drink with friends and thus adhere to social norms. As there is little existing research investigating the individual components of the social self-efficacy, negative social events, and drinking 
relationship, examining this relationship will be one research question addressed in this dissertation. Thus,

Research Question 1: What is the relationship between social self-efficacy and drinking with others?

Research Question 2: Does social self-efficacy moderate the relationship between negative social events and drinking? If so, what is the direction of this relationship? 


\section{CHAPTER VIII}

\section{Methods}

\section{Study Overview}

This dissertation is a secondary analysis of data collected as part of a larger study regarding the impact of cross-cultural adjustment on well-being and health, which was directed by Mo Wang, Ph.D., and Cynthia D. Mohr, Ph.D., and funded by the Medical Research Foundation of Oregon. I assisted Drs. Wang and Mohr by serving as Project Manager and was involved in all aspects of the project. My role as project manager included working with Drs. Wang and Mohr to develop and implement study protocol, creating the internet survey instrument and survey maintenance, creating participant tracking systems, supervising the survey translation process (from English into Japanese, and the back-translation process), recruiting participants and conducting participant orientation sessions, disbursement of participant compensation, correspondence with participants, and supervision of undergraduate research assistants.

\section{Procedure}

Recruitment. Participants were recruited via academic contacts in Tokyo, Japan and Portland, Oregon, as well as through referrals from international students currently studying in the United States. Participants in Portland, Oregon were participating in an international study abroad program through Waseda University in Tokyo, Japan. The one-year study abroad program included Waseda University students who had been selected to study at Portland State University. Portland State University and Waseda 
University are sister universities and the exchange program is well-developed in that there is formal, scheduled academic and social programming for visiting students. Waseda University students arrive to Portland in September, and are enrolled at Portland State University throughout the academic school year, ending in June.

International students were recruited via an email sent directly to Waseda University students studying at Portland State University, via the International Student Office at Portland State University. Interested participants also recruited additional Waseda University students studying at Portland State University by word-of-mouth. Students studying at Waseda University in Tokyo, Japan were recruited via a faculty member at Waseda University. This professor recruited students from her classes and by word-of- mouth. Each method of recruitment included providing potential participants with information regarding the study and inviting them to participate in an orientation session.

The students studying in Portland attended one of five group orientation sessions, where I presented an overview of the study and provided in-depth training on completing the web-survey. Participants signed informed consent at these orientation sessions. These orientation sessions were conducted in English, although a native Japanese speaker also attended the sessions in order to provide any necessary translation. The students who were studying in Tokyo participated in a one-on-one orientation over the phone. A native Japanese speaker, who was a research assistant for this project, conducted these orientation sessions in Japanese. I provided supervision for these phone calls. Participants in Japan met with our Waseda 
University contact to complete informed consent. The informed consents were mailed to our research lab at Portland State University.

In order to be eligible to participate, participants needed to be of legal drinking age (21 in the United States; 20 in Japan). Participants had to be full-time college students. There were no other eligibility requirements. No interested individuals were excluded based on these eligibility criteria, although the recruitment advertisements were clear in stating the eligibility requirements, so it is likely that interested individuals who did not meet these basic eligibility requirements did not express interest, and thus were not included in orientation sessions.

One participant signed an informed consent, but then chose not to participate in the study, before completing the initial assessment. This participant did not provide information for why he decided not to participate in the study.

Initial Assessment. Following the orientation sessions, participants were contacted via email to access the initial questionnaire. The initial assessment questionnaire was an online survey (i.e., Questionpro), accessible only to individuals who received a unique invitation to complete the survey. The initial assessment took approximately one hour to compete; participants were given one week to complete the initial assessment. The initial assessment was comprised of multiple psychosocial questionnaires, including individual difference measures such as cross-cultural adjustment, social self-efficacy and drinking refusal self-efficacy (Figure 4). Participants were not allowed to continue to the daily diary portion of the study if they had not signed the informed consent and completed the initial assessment procedure. 
Two participants completed the initial assessment, but did not participate in the daily diary portion of the study.

Daily Interview. Participants started the daily portion of the study on the same day of the week, at different times throughout the year. Participants were sent a daily email to their email account reminding them to fill out the survey each day. Participants were able to complete the daily surveys between 3-8pm each day. This time period was chosen because it coincides with a time period that marks the end of a students' workday and the beginning of the evening (where drinking is more likely to occur; Mohr et al., 2005). Each day, participants answered questions regarding the previous evening's activities, the current day's activities, and possible outcomes of drinking alcohol that evening. Although the focus of the current proposed secondary analysis is on alcohol consumption, each day, participants answered questions regarding alcohol consumption and other activities, such as physical health, work stress, school stress, daily goals, and significant events. Thus, participants were not necessarily aware that this was a study on alcohol consumption. Once a survey was submitted, participants were unable to go back to the survey and change their answers, thus eliminating any potential back filling of surveys. This interview schedule is consistent with the interval-contingent method of ecological momentary assessment as described in Chapter VI of this proposal (Reis \& Gable, 2000). Figure 4 depicts when key measures were assessed.

Debriefing Interview. At the end of the 30 days participants completed a webbased debriefing questionnaire, similar to the initial assessment survey. Again, a 
battery of psychosocial measures was included in the debriefing interview. The debriefing interview took approximately 45 minutes to complete and participants had one week, following the completion of the daily interview portion of the study, to complete the debriefing questionnaire. Participants were paid per survey, with weekly bonuses for completing all of the surveys during the week, in addition to a payment for completing the initial and debriefing questionnaires. Participants who completed at least $75 \%$ of the daily surveys were entered into a lottery to win one of eight cash prizes. In total, participants were able to earn up to $\$ 65$ and win a lottery prize of $\$ 75$.

\section{Participants}

Participants were 16 Japanese college students at a large Japanese university and 41 Japanese international students studying at a university in the northwestern U.S. Japanese local students (Japanese students studying in Japan) were eligible to participate if they were 20 years old or older (the legal drinking age in Japan is 20). Japanese international students (Japanese students who were studying abroad in the U.S.) were eligible if they were 21 years or older. Two participants were not included in the analyses, as they were outside of the traditional college student age range (ages 45 and 69). Of the remaining participants, the average age was $23(\mathrm{SD}=2.96)$. Women comprised $79 \%$ of the sample.

The participants who were also international students studying in Portland were participating in an established study abroad program through a partnership between Waseda University (their home school) and Portland State University. These universities are considered "sister universities," meaning that a reputable relationship 
exists between the two universities and a formalized program of study exists.

Specifically, these students arrived in the U.S. as a cohort, attended classes together and lived in close proximity to each other. Social events for this cohort of students were also organized through the Waseda University office located on the Portland State University campus. Thus, although there was a shift in geographical context for this group, the social context was relatively familiar.

Measures

All measures were presented to participants in Japanese. In order to ensure reliability of translation, all scales were initially translated from English into Japanese by one translator, and then back into English by a second translator. The original measure and the back-translated measure were compared and a percent agreement was calculated for each battery and all percentages were greater than $93 \%$. Instances where there were discrepancies between the original English version of the measure and the back-translated version were resolved via a discussion among three research assistants who were fluent in Japanese and English.

\section{Initial Assessment Measures}

Between-person measures of self-efficacy were assessed at the initial assessment. These are trait measures of social and drinking refusal self-efficacy.

Drinking Refusal Self-Efficacy. The 19-item Drinking Refusal Self-Efficacy

Questionnaire (Oei, Hasking, \& Young, 2005) was administered to determine individuals' ability to decline an alcoholic beverage in certain circumstances. Participants were instructed to indicate how sure or unsure they were that they would 
drink in 19 different situations. There are three subscales: social pressure (SP), emotional relief (ER), and opportunistic drinking (OD). Situations include "When I come home from school," "When I am at dinner," and "When someone offers me a drink." The responses range from 1 (I am very sure I would drink) to 6 (I am sure that I would not drink). Higher scores indicate a greater ability to refuse drinking in certain situations. This scale has been shown to have good construct and concurrent validity in predicting alcohol consumption (Oei et al., 2005). Correlations between the subscales indicated that all three scales were significantly correlated to each other (social pressure and emotional relief, $r=.55, p<.01$; social pressure and opportunistic drinking $r=.54, p<.01$; opportunistic drinking and emotional relief, $r=.79, p<.01$ ). Furthermore, a scree plot identified one overall factor of drinking refusal self-efficacy. For these reasons, a total scale score of drinking refusal self-efficacy will be used in the data analyses. Within this sample, this scale was internally consistent, $\alpha=.94$.

Social Self-efficacy. The Social Self-efficacy Questionnaire (Gong \& Fan, 2006) was administered to determine individuals' social self-efficacy. This eight-item scale measures individuals' beliefs about their confidence that they could properly engage in social situations. Participants rated their confidence on a scale of 1 (No confidence) to 5 (complete confidence). Example items include "Ask a group of people who are planning to engage in a social activity (e.g., go to a movie) if you can join," and "Get invited to a party that is being given by a prominent or popular individual." This scale has been shown to have good internal consistency ( $\alpha=.89$; Gong \& Fan, 2006). Within this sample, this scale was internally consistent $(\alpha=.88)$. 


\section{Daily Interview Measures}

Within-person measures were assessed daily during the course of the 30-day diary study. These measures include daily negative social events, alcohol outcome expectancy desirabilities, and daily consumption. Participants completed the daily portion of the survey each day between $3-8 \mathrm{pm}$, however, participants were asked to recall events and drinking during recent reference points. Specifically, participants were asked to recall daily negative social events that occurred from the time they woke up until the time that they completed the diary. Participants were asked to report their drinking from the night before (i.e., after the completed the diary until they went to bed). Finally, participants were asked to report their current alcohol outcome expectancy desirabilities. Figure 5 shows the daily interview timeline; further description of the within-person measures follow.

Daily Negative Social Events. Each day participants rated the occurrence of daily events using a 14-item daily event checklist (adapted from Gable, Reis, \& Elliot, 2000). Participants were asked to indicate whether or not events from a checklist occurred that day. If events occurred, participants were asked to rate how desirable the event was on a scale ranging from 1 (extremely undesirable) to 7 (extremely desirable). Events that were appraised as 1-3 on the scale were considered negative events, whereas events appraised as 5-7 on the scale were considered positive events. Examples of events are "Had a disagreement or conflict with friend(s) or girlfriend/boyfriend," and "Did something special for friend(s) or girlfriend/boyfriend that was appreciated." 
Alcohol Outcome Expectancy Desirability. Each day participants reported the desirability of four alcohol outcome expectancies (Armeli et al., 2005) on a scale of 1 (not at all desirable) to 7 (very desirable). Examples of items include: "A sense of carelessness from drinking alcohol," "A pleasant feeling from drinking alcohol with friends.” Armeli et al. (2005) found this scale to have good internal consistency $(\alpha=.93)$. Internal consistency for this scale was measured at three points throughout the 30 days (Day 7, Day 14, and Day 21). For this sample, this scale was internally consistent ( $\alpha=.78, \alpha=.75, \alpha=.72$, respectively).

Daily Consumption. Participants reported the previous night's consumption each day. Specifically, participants were asked to report the total quantity of alcohol they consumed at home (or in their dorm room) and away from home. Using a checklist with response options ranging from one to greater than 15 , participants reported how many drinks they had at home while drinking alone and how many drinks they consumed while at home interacting with others. Similarly, participants reported how many drinks they consumed away from home alone and away from home with other people. These reports were based on standardized measurements of alcohol content $(1$ glass $=500 \mathrm{~mL}$ glass of beer, $148 \mathrm{~mL}$ glass of wine, $355 \mathrm{~mL}$ wine cooler, $45 \mathrm{~mL}$ shot, or in a cocktail; Wechsler, Nelson, Lee, Seibring, Lewis, \& Keeling, 2002). Daily consumption was calculated by summing the total number of drinks each individual drank with others (either away from home or at home) with larger numbers indicating greater number of drinks consumed. 


\section{Data Analyses}

In the following sections, I discuss the process I used to analyze the data. First, I discuss the between-group comparisons I conducted to examine whether or not there were significant group differences in drinking with others between students who were studying in Japan and students who were studying in the U.S. Next, I discuss the process I used for variable creation. Then, I discuss the prevalence and type of missing data. Finally, I discuss the data structure, including day of the week trends in drinking with others.

\section{Between-Group Comparisons}

The first step was to determine whether there were differences between groups in the outcome variables of interest (drinking with others and alcohol outcome expectancy desirability), as the data was collected from Japanese international students and Japanese local students. In order to determine whether there were significant group differences on each outcome variable, I used a t-test for each variable, comparing the two groups to each other. The t-test results showed no significant differences between local and international students on AOED $[t(24.59)=1.74, p=.09]$ nor in the amount of drinking with others $[t(14.74)=1.56, p=.14]$. Based on the nonsignificant results from the t-tests, there were no significant group differences. Thus,

for the remainder of the analyses, I did not control for group membership.

\section{Variable Creation}

Centering. Centering variables creates a meaningful zero point, and eases interpretation. For within-person variables, such as daily negative social events and 
daily alcohol outcome expectancy desirability, centering the variable allows for the interpretation of an average score for each individual to be obtained (Enders \& Tofighi, 2007). By centering these variables, I am able to interpret changes in an individual's score, based on their average level of daily negative social events and daily alcohol outcome expectancy desirability. For example, I can examine changes in consumption levels when there is a unit increase in daily negative social events, compared to the average number of daily negative social events an individual experiences, instead of days when one does not experience negative social events. Centering variables does not change the scale, nor does it change the regression coefficient; the regression coefficient is interpreted as the effect variable $\mathrm{X}$ has on mean levels of variable Y (Enders \& Tofighi, 2007). For the current analysis, I personcentered the within-person variables (negative social events and alcohol outcome expectancy desirability). Thus, when interpreting the multi-level regression coefficient for negative social events predicting drinking with others, the coefficient can be interpreted as positive or negative variations from an individual's average level of daily negative social events. The same is true for the coefficient for alcohol outcome expectancy desirability predicting drinking with others.

For the between-person variables (Level-2 variables), social self-efficacy and drinking refusal self-efficacy were grand mean centered; the grand mean is the overall average of scores for all individuals in the study. By grand mean centering the Level-2 variable, I can interpret the intercept as the expected value of the outcome variable (drinking) when all predictors are at their mean levels, or in this case, on an average 
day for the participants (Hoffman, 1998; Hox, 2002). This eases interpretation of the regression coefficients. Additionally, multicolinearity, due to the interaction terms, is reduced (Enders \& Tofighi, 2007).

Lagged Variables. This dissertation examines the temporal relationships between daily negative social events, daily alcohol outcome expectancy desirability, and drinking. As previously discussed, each day, participants reported their previous night's alcohol consumption, the negative social events that occurred the day, and the current alcohol outcome expectancy desirability. In order to create a temporal ordering of variables, whereby daily negative social events and alcohol outcome expectancy desirability predict subsequent drinking, I created lagged variables; daily negative social events and alcohol outcome expectancy desirability reported on day $t$ were used to predict drinking reported on day $t+1$. In order to create the lagged variables, I used syntax in SPSS to compute lagged variables for the daily negative social events and daily alcohol outcome expectancy desirability. This syntax shifted the data for these variables down one cell in SPSS. Shifting the variable down one cell in SPSS results in adding one instance of missing data per participant, as their last report of negative social events and alcohol outcome expectancy desirability is not used to predict drinking outcomes.

Interaction Terms. Hypothesis 5 and Research Question 2 proposed a crosslevel interaction between drinking refusal self-efficacy and social self-efficacy, and daily negative social events. In order to evaluate these cross-level moderators, I created an interaction term for each variable. First, as I previously discussed, I 
centered the predictor variables in SPSS and then created the interaction terms in HLM in order to enhance interpretation (Aiken \& West, 1991).

Missing Data. Multi-level modeling allows for an unequal number of observations (Snijders \& Bosker, 1999). For daily interviews, where participants are asked to answer a survey each day, the likelihood of missing data exists; it is unlikely that every person completed every interview. The result is the potential for missing data. According to Little and Rubin (1987), there are three types of missing data: nonignorable missing (NIM), missing completely at random (MCAR), and missing at random (MAR). Data that is NIM are data that are systematically missing (Little \& Rubin, 1987). In other words, data that are NIM have intentionally been left unanswered by participants. An example is if you are surveying individuals about their socioeconomic status and well-being and you find a large amount of missing data for income level. If you look at the missing data cells for income level and there is an association between the missing data and the well-being variable, you may have found that participants intentionally did not answer questions regarding income level based on their level of well-being (e.g., people with higher well-being may have felt uncomfortable listing their income level). Information must be obtained and data must be carefully looked at in order to determine accurate parameter estimates (Allison, 2002).

Data that is MCAR assumes that missing data is completely independent from other variables. That is, participants may have unintentionally skipped a question, or not realized they did not answer a question. Similarly, perhaps an electronic 
malfunction occurred with the web-based survey and participants were unable to answer the question. In comparison, MAR is a less restrictive type of missing data; MAR assumes that information regarding the missing data can be obtained from other information in the data set.

The type of missing data most frequently found in multi-level modeling is MAR (Hox, 2002). Attrition in multi-level modeling contributes to MAR data. This data is not MCAR because some individuals may be more likely to discontinue their participation than others. According to Hox (2002), the amount of data obtained for multi-level modeling allows for researchers to assess missing data. I used several techniques to determine the amount of missing data, and understand possible patterns of missing data.

First, in order to determine the amount of missing data, I first assessed overall compliance. I computed compliance by determining the total possible number of surveys that could be completed ( $\mathrm{n}=30$ for each participant) and then counted the total number of surveys that were actually completed. I divided the total number of surveys that were actually completed by the total possible number of surveys. The total number of surveys completed was 1195 . The total possible number of interviews was 1650. Overall compliance was $72 \%$. I then investigated the missing data for individual participants. Thirty-three participants completed $70 \%$ of the interviews; four participants only completed $30 \%$ of the surveys.

I also investigated the amount of missing data for daily negative social events and alcohol outcome expectancy desirability. Thirty-one participants completed $70 \%$ 
or more of the negative social events AOED questions. Five participants completed $30 \%$ or less of the negative social events and six participants completed $30 \%$ or less of the AOED. In order to determine whether the individuals who completed less than $30 \%$ of the data differed (in terms of the number of negative social events, alcohol outcome expectancy desirability and drinking with others) from those who completed greater than $30 \%$ of the data, I investigated the means for each variable, for each group (those who completed more than $30 \%$ and those who completed less than $30 \%$ ). Using independent samples t-tests, the results showed no significant group differences in the number of negative social events $[t(53)=-.48, p=.63]$, alcohol outcome expectancy desirability $[t(53)=1.62, p=.11]$, or for drinking with others $[t(48)=-.58, p=.57]$. Thus, it appears that missing data is not related to the number of negative social events, alcohol outcome expectancy desirability or the number of drinks with others, indicating that missing data is not systematic and can be considered MAR.

Next, I assessed missing data by each predictor variable. Using dummy coding, I coded missing data and non-missing data, assigning values of zero to non-missing data and values of one to missing data. I then regressed the predictor on each dummy code, in separate regression equations. A significant relationship was not found for drinking with others $[F(1,48)=.05, p=.82]$, alcohol outcome expectancy desirability $[F(1,53)=2.80, p=.10]$ or negative social events $[F(1,53)=1.10, p=.30]$. Finally, I regressed drinking with others on the dummy coded variable for missing data for negative social events and for alcohol outcome expectancies. Missing data on the negative social events variable did not significantly predict drinking with others 
$[F(1,48)=.13, p=.72]$. Likewise, missing data on the alcohol outcome expectancy desirability variable did not predict drinking with others $[F(1,48)=.02, p=.90]$. Thus, no significant regressions were found. The missing data was not significantly related to drinking with others. Although there was evidence of missing data, the data does not seem to be systematically missing.

Finally, an additional source of missing data can originate from participants starting the daily diary late, or by quitting the study early. Based on the rotating start date for participants, participants did not start the study late. If participants missed starting the daily diary portion on the start date (Tuesday), they were able to start the diary the following week. Thus, no missing data is attributable to participants starting the protocol late. Although no specific requests were received from participants to be removed from the study, there were four participants who stopped completing surveys after day 23 (the last week of the interview time period).

When examining the missing data, it is important to remember that lagging of the within-person predictors contributes to missing data. Despite missing data, multilevel modeling does not assume an equal number of data points for each person. Furthermore, I used HLM 6.0 for my analyses, which analyzes missing data and weights data according to the number of available cases (i.e., data from individuals with fewer observations will be weighted less than individuals with more observations). 


\section{Data Structure}

The data were collected using daily interviews, and thus are hierarchically structured with 30 sets of negative social events and alcohol outcome expectancy desirability responses and reports of consumption for each of the 55 participants. That is, each participant had the opportunity to answer negative social events, desirability, and consumption-related questions each day for 30 days. As previously mentioned, there is missing data and thus, this is an unbalanced data set. In order to address the structure of the data, I analyzed the data using multi-level modeling, which allows for comparisons to be made between persons (at Level-2) and within person (at Level-1). I used HLM v6.0 (Raudenbush et al., 2000) to analyze the data because it allows for unbalanced, hierarchically structured data. In HLM I specified two equations, a within-person regression model (Level-1 variables) and a between-person regression model (Level-2 variables). The within-person equation included a within-person outcome (i.e., drinking with others), which was modeled as a function of the Level-1 predictors (i.e., daily negative social events, and daily alcohol outcome expectancy desirability). The between-person equation (Level 2) included the intercepts and slopes from Level 1 as a function of the between-person predictors (i.e., drinking refusal self-efficacy and social self-efficacy).

Trends and Serial Dependency. Following West and Hepworth's (1991) warning concerning temporally ordered data and findings showing day-of-the-week trends in alcohol consumption (e.g., Argeriou, 1975; Carney et al., 1998), I created six orthogonal dummy variables to model day of the week. Data collected via daily 
process methodology is susceptible to trends, such as differences between weekdays and weekends. Specifically, alcohol consumption may be higher on weekends compared to weekdays. Indeed, previous work has documented these trends (Argeriou, 1975; Armeli et al., 2005). These day of week covariates were included in the analyses and were modeled as fixed effects (Bryk \& Raudenbush, 1992). Argeriou (1975) suggested that Tuesday is an ideal day to hold constant for drinking variables because it does not represent the day where consumption is lowest, nor does it represent a day where consumption is highest. In order to select a day to hold constant, it is important to pick a day that represents an "average" day in terms of drinking. Argeriou (1975) and Carney et al. (2000) have found that Tuesday represents an average day for drinking. As a first step in data analysis, I regressed alcohol consumption on the day of week covariates. There were significant coefficients, indicating that there are day of week effects. Indeed, drinking was significantly higher on Thursdays $(\beta=1.28, p<.01)$, Saturdays $(\beta=1.58, p<.01)$, and Sundays $(\beta=1.42, p<.01)$, compared to Tuesdays. I controlled for the day of the week effects in all of the subsequent analyses.

Finally, the outcome variable, number of drinks consumed with others, is a count variable, and includes a larger proportion of zeros, compared to other numbers. Following guidelines in the literature, HLM v6.0 is an appropriate statistical package to handle such a distribution and allows for a Poisson distribution with a log-link function (Raudenbush \& Bryk, 2002; Armeli et al., 2005). 


\section{Chapter IX}

Results

\section{Descriptive Information}

On average, participants consumed alcohol with others on approximately five days throughout the 30-day study $(\mathrm{M}=5.13, \mathrm{SD}=3.72)$. On days when participants reported drinking alcohol with others, participants drank almost five drinks (M=4.65, $\mathrm{SD}=3.70)$. Table 1 shows the between-person descriptive statistics. Indeed, participants most commonly consumed alcohol with others on Sundays $(\mathrm{M}=1.15$, $\mathrm{SD}=2.58)$, Thursdays $(\mathrm{M}=1.22, \mathrm{SD}=3.50)$, and Saturdays $(\mathrm{M}=1.59, \mathrm{SD}=3.29)$. On average, participants consumed the least on Wednesdays $(\mathrm{M}=.44, \mathrm{SD}=1.73)$.

Throughout the study, participants reported experiencing an average of 1.27 $(\mathrm{SD}=2.51)$ negative social events per day. Participants reported experiencing the highest number of negative social events on Wednesdays ( $\mathrm{M}=1.24, \mathrm{SD}=2.99)$, Fridays $(\mathrm{M}=1.24, \mathrm{SD}=2.71)$, and Saturdays $(\mathrm{M}=1.15, \mathrm{SD}=2.37)$. A significant correlation between gender and negative social events was found $(\mathrm{r}=-.29,<.05)$, indicating that men reported experiencing more negative social events than women (Table 1).

As a first step to conducting the hierarchical models, I wanted to ensure that the intraclass correlation (ICC) was sufficiently high enough to warrant the necessity of accounting for group membership (in this case the group is the participant) in the models (Snijders \& Bosker, 1999). To calculate the ICC, the following equation is used:

Variance in intercept/(residual variance + variance in intercept) 
The resulting number can be interpreted as a percentage of the amount of variation that is attributable to group membership. The ICC for negative social events was .70, indicating that $70 \%$ of the variance in negative social events was due to group differences. Thus, the independence assumption of ordinary regression is violated the use of hierarchical linear modeling is further warranted (e.g. Snijders \& Bosker, 1999) Participants reported relatively low alcohol outcome expectancy desirability $(\mathrm{M}=1.68, \mathrm{SD}=.69)$. Specifically, the scale for alcohol outcome expectancy desirability ranged from one to seven; thus, an average of 1.68 is low. A significant and negative correlation was found between gender and AOED ( $\mathrm{r}=-.46, p<.01)$, indicating that men also reported higher AOED. Alcohol outcome expectancy desirability was significantly and positively correlated with negative social events $(\mathrm{r}=.30, p<.05)$. The correlation indicates that a higher number of negative social events is associated with higher alcohol outcome expectancy desirability. I calculated the ICC for AOED in order to determine the amount of variability due to group membership. The ICC was .42 , indicating the $42 \%$ of the variance in AOED is attributed to group membership. This means that there was greater variation between alcohol outcome expectancy desirability scores selected from two different individuals than randomly selected scores. In other words, individuals widely varied from each other in terms of desirability, and it is, therefore, important to account for group membership (here, the individual).

For the between-person measures, participants reported a moderate level of drinking refusal self-efficacy $(M=4.07, S D=.90)$, thus indicating that they felt 
relatively confident about their ability to refuse alcohol. As expected, drinking refusal self-efficacy was significantly and negatively correlated to drinking with others ( $\mathrm{r}=-$ $.39, p<.01)$. Thus, individuals who had higher drinking refusal self-efficacy also drank less with others. Similarly, participants reported moderate social self-efficacy $(\mathrm{M}=2.82, \mathrm{SD}=.77)$, indicating that they had reasonable confidence in their ability to engage in social relationships. Social self-efficacy was positively correlated with negative social events $(\mathrm{r}=.39, p<.01)$. Individuals who had high social self-efficacy also reported a greater number of negative social events.

\section{Hypothesis Testing}

In the following section, I discuss the hypothesis testing. A summary of each hypothesis and research question, as well as the result for each hypothesis or research question is found in Table 9.

\section{Hypothesis 1: Negative Social Events Predicting Drinking with Others}

Hypothesis 1 posited that negative social events would predict drinking with others. That is, on days when individuals experienced increases in negative social events, they would report drinking more with others that evening.

The following model was used to test this hypothesis:

$$
\begin{aligned}
& \text { Drink }_{\mathrm{it}}=\beta_{0 \mathrm{i}}+\beta_{1 \mathrm{it}}(\text { NEGEVENT })+\beta_{2 \mathrm{it}}(\text { Mon })+\beta_{3 \mathrm{it}}(\text { Wed })+\beta_{4 \mathrm{it}}(\text { Thurs })+\beta_{5 \mathrm{it}} \\
& (\text { Fri })+\beta_{6 \mathrm{it}}(\text { Sat })+\beta_{7 \mathrm{it}}(\text { Sun })+\mathrm{e}_{\mathrm{it}}
\end{aligned}
$$

In this model, DRINK is the number of drinks for each person $i$ on day $j$. The random intercept for person $i$ is $\beta_{0 \mathrm{i}}$, or the predicted value of daily consumption when all of the predictors are zero. As negative social events was person-centered, a zero 
represents the individual's average level of negative social events, not actually a day when the individual experiences no negative social events. That is, $\beta_{0 \mathrm{i}}$ can be considered the individual's average level of drinking with others. The random error is $\mathrm{e}_{\mathrm{it}}$. The association of negative social events on drinking with others is the $\beta_{1 \mathrm{it}}$ coefficient. The other coefficients represent the association of each day of the week on drinking with others. As previously discussed, these were modeled as fixed effects. When examining the fixed day of week effects, the results of this model show that drinking on Sundays, Thursdays, and Saturdays was significantly higher, compared to Tuesdays. Table 2 provides all of the coefficients for the model. The results showed that $\beta_{1 \mathrm{it}}$ was significant at the $\alpha=.05$ level $\left(\beta_{1 \mathrm{it}}=.23\right)$. As hypothesized, a positive relationship was found between negative social events and drinking with others, such that on days when individuals experienced more negative social events, relative to days when they experienced fewer negative social events, individuals reported greater drinking with others. Recent alcohol research uses a Poisson sampling distribution with a log-link function (e.g. Armeli, Mohr, Todd, Tennen et al., 2005). For example, for negative social events, we can used the following equation to determine a meaningful interpretation of the negative social event coefficient:

$$
\operatorname{Exp}\left(\beta_{1 i t}\right)=\text { drinks with other }
$$

Thus, $\operatorname{EXP}(.23)=1.26$. We can determine then that for every one-unit increase in negative social events, there is a $26 \%$ increase in the number of drinks consumed with others. Furthermore, when examining the variance component for negative social events, we see that there is significant variability in negative social events $(u=.05$, 
$p<.05)$. In sum, Hypothesis 1 was supported; on days when individuals experienced greater negative social events, individuals subsequently drank more with others.

Hypothesis 2: Negative Social Events Predicting Alcohol Outcome Expectancy

\section{Desirability}

Hypothesis 2 stated that alcohol outcome expectancy desirability would be higher on days with greater negative social events compared to days with fewer negative social events.

The following model was used to test this hypothesis:

$$
\begin{aligned}
& \operatorname{AOED}_{\mathrm{it}}=\beta_{0 \mathrm{i}}+\beta_{1 \mathrm{it}}(\mathrm{NEGEVENT})+\beta_{2 \mathrm{it}}(\text { Mon })+\beta_{3 \mathrm{it}}(\mathrm{Wed})+\beta_{4 \mathrm{it}}(\text { Thurs })+\beta_{5 \mathrm{it}} \\
& (\text { Fri })+\beta_{6 \mathrm{it}}(\text { Sat })+\beta_{7 \mathrm{it}}(\mathrm{Sun})+\mathrm{e}_{\mathrm{it}}
\end{aligned}
$$

In this model, $\mathrm{AOED}_{\mathrm{tj}}$ is the daily desirability of alcohol outcome expectancies for person $i . \beta_{0 \mathrm{i}}$ is the random intercept, or the desirability for an individual's average level of negative social events, and $e_{i t}$ is the random error. The coefficient $\beta_{1 \text { it }}$ is the effect of negative social events on alcohol outcome expectancy desirability. As seen in Table 3, the coefficient for negative social events $\left(\beta_{1 \mathrm{it}}\right)$ was not significant at the $\alpha=.05$ level. Thus, negative social events did not significantly predict alcohol outcome expectancy desirability. The variance component for negative social events was also not significant. In sum, Hypothesis 2 was not supported, negative social events did not predict alcohol outcome expectancy desirability. Hypothesis 3: Alcohol Outcome Expectancy Desirability and Drinking with Others

Although negative social events did not significantly predict alcohol outcome expectancy desirability, it was still possible that alcohol outcome expectancy 
desirability predicted drinking with others. I investigated this relationship, testing the following hypothesis that drinking with others would be greater on days when individual's reported increases in the desirability of alcohol outcome expectancies. This hypothesis was tested using the following model:

$$
\begin{aligned}
& \text { DRINK }_{\mathrm{it}}=\beta_{0 \mathrm{i}}+\beta_{1 \mathrm{it}}(\text { AOED })+\beta_{2 \mathrm{it}}(\text { Mon })+\beta_{3 \mathrm{it}}(\text { Wed })+\beta_{4 \mathrm{it}}(\text { Thurs })+\beta_{5 \mathrm{it}}(\text { Fri })+ \\
& \beta_{6 \mathrm{it}}(\text { Sat })+\beta_{7 \mathrm{it}}(\text { Sun })+\mathrm{e}_{\mathrm{it}}
\end{aligned}
$$

In this model, DRINK $\mathrm{it}_{\mathrm{it}}$ is the daily consumption for person $i$ when AOED is zero. The random intercept is $B_{01}$ and $e_{i t}$ is the random error. The effect of alcohol outcome expectancy desirability on drinking is found in the coefficient $\beta_{0 \mathrm{i}}$. As seen in Table 4 , the results showed a significant relationship between alcohol outcome expectancy desirability and drinking with others $\left(\beta_{1 \mathrm{it}}=.98, p<.001\right)$. Thus, on days when alcohol outcome expectancy desirability was higher, individuals drank more with others, compared to days with lower alcohol outcome expectancy desirability. Again, by exponentiating $\beta_{1 \mathrm{it}}$, we can meaningfully interpret the increase in the number of drinks. For every one-unit increase in alcohol outcome expectancy desirability, drinking with others increased by 2.66 drinks. Further, the variance component for alcohol outcome expectancy desirability was significant $(\mathrm{u}=.49, p<.001)$, indicating significant variability in alcohol outcome expectancy desirability. Thus, Hypothesis 3 was supported; drinking with others was significantly higher on days when individuals reported increases in alcohol outcome expectancy desirability. Hypothesis 4: Drinking Refusal Self-Efficacy Predicting Drinking with Others 
After determining the nature of the within-person relationships, I examined the between-person relationships. First, I examined whether drinking refusal self-efficacy (DRSE) significantly predicted drinking with others. Specifically, Hypothesis 4 stated that individuals with greater drinking refusal self-efficacy, or the ability to decline drinks, would report less drinking with others. In order to test this hypothesis, I used the following model:

$$
\begin{aligned}
& \operatorname{Drink}_{\mathrm{it}}=\beta_{0 \mathrm{i}}+\beta_{1 \mathrm{it}}(\text { Mon })+\beta_{2 \mathrm{it}}(\text { Wed })+\beta_{3 \mathrm{it}}(\text { Thurs })+\beta_{4 \mathrm{it}}(\text { Fri })+\beta_{5 \mathrm{it}}(\text { Sat })+\beta_{6 \mathrm{it}} \\
& (\text { Sun })+\mathrm{e}_{\mathrm{it}} \\
& \quad \beta_{0 \mathrm{i}}=\gamma_{00}+\gamma_{01}(\text { DRSE })+\mathrm{u}_{0 \mathrm{i}}
\end{aligned}
$$

In this equation, $\beta_{0 \mathrm{i}}$ is the random intercept of drinking. The level 1 intercepts and slopes are modeled as a function of the between-person factor of drinking refusal selfefficacy. As outlined in Table 5, the results indicated that drinking refusal self-efficacy significantly and negatively predicted drinking with others $\left(\gamma_{01}=-.53, p<.001\right)$.That is, for every one-unit increase in drinking refusal self-efficacy, drinking with others decreased by an average of .58 drinks. Thus, Hypothesis 4 was supported; as hypothesized, individuals who had higher levels of drinking refusal self-efficacy reported lower levels of drinking with others.

Hypothesis 5: Drinking Refusal Self-Efficacy as a Moderator of the Negative Social Events-Drinking with Others Relationship

Hypothesis 1 was confirmed; individuals drank more with others following increases in negative social events. Moreover, Hypothesis 4 was also confirmed, 
drinking refusal self-efficacy has a significant and negative relationship to drinking with others. Thus, I further investigated the relationship between drinking refusal selfefficacy and drinking with others by testing the cross-level moderating relationship of drinking refusal self-efficacy on negative social events and drinking with others. In order to test this hypothesis, I used the following model:

$$
\begin{gathered}
\text { Drink }_{\mathrm{it}}=\beta_{0 \mathrm{i}}+\beta_{1 \mathrm{it}}(\text { Mon })+\beta_{2 \mathrm{it}}(\text { Wed })+\beta_{3 \mathrm{it}}(\text { Thurs })+\beta_{4 \mathrm{it}}(\text { Fri })+\beta_{5 \mathrm{it}}(\text { Sat })+\beta_{6 \mathrm{it}} \\
(\text { Sun })+\beta_{7 \mathrm{it}}(\text { NEGEVENT })+\mathrm{e}_{\mathrm{it}} \\
\beta_{0 \mathrm{i}}=\gamma_{00}+\gamma_{01}(\text { DRSE })+\mathrm{u}_{0 \mathrm{i}} \\
\beta_{7 \mathrm{i}}=\gamma_{10}+\gamma_{11} \text { (DRSE) }+\mathrm{u}_{0 \mathrm{i}}
\end{gathered}
$$

In this equation, $\beta_{0 \mathrm{i}}$ is the random intercept of drinking. The level 1 intercepts and slopes are modeled as a function of the between-person factor of drinking refusal selfefficacy. The results for this model are included in Table 4 and indicate that drinking refusal self-efficacy was a marginally significant moderator of the negative social events-drinking with others relationship $\left(\gamma_{11}=-.09, p=.08\right)$. For this model, the variance component for negative social events was not significant. Thus, Hypothesis 5 was marginally supported, but was not significant. Drinking refusal self-efficacy did not significantly moderate the relationship between negative social events and drinking with others.

Research Question 1: Social Self-Efficacy Predicting Drinking with Others

After investigating the relationship between drinking refusal self-efficacy and drinking with others, I investigated the relationship between a second type of self- 
efficacy, social self-efficacy. Specifically, I wanted to investigate the relationship between social self-efficacy and drinking with others. In order to determine the relationship between social self-efficacy (SSE) and drinking, I used the following model:

$$
\begin{aligned}
& \operatorname{Drink}_{\mathrm{it}}=\beta_{0 \mathrm{i}}+\beta_{1 \mathrm{it}}(\text { Mon })+\beta_{2 \mathrm{it}}(\text { Wed })+\beta_{3 \mathrm{it}}(\text { Thurs })+\beta_{4 \mathrm{it}}(\text { Fri })+\beta_{5 \mathrm{it}}(\text { Sat })+\beta_{6 \mathrm{it}} \\
& (\text { Sun })+\mathrm{e}_{\mathrm{it}} \\
& \quad \beta_{0 \mathrm{i}}=\gamma_{00}+\gamma_{01}(\mathrm{SSE})+\mathrm{u}_{0 \mathrm{i}}
\end{aligned}
$$

In this equation, $\beta_{0 \mathrm{i}}$ is the random intercept of drinking. The level 1 intercepts and slopes are modeled as function of the between-person factor of social self-efficacy. As seen in Table 7, the results indicated a significant and positive relationship between social self-efficacy and drinking with others $\left(\gamma_{01}=.32, p<.05\right)$. Thus, individuals with higher social self-efficacy also report higher levels of drinking with others, relative to individuals with lower social self-efficacy. Thus, social self-efficacy significantly and positively predicts drinking with others.

Research Question 2: Social Self-Efficacy as a Moderator of the Negative Social Event-Drinking with Others Relationship

Given the significant and positive relationship found between negative social events and drinking with others, and the significant findings for social self-efficacy positively predicting drinking with others, a logical next step was to investigate social self-efficacy as a moderator of the negative social event/drinking with others relationship. This relationship was examined using the following model: 


$$
\begin{gathered}
\text { Drink }_{\mathrm{it}}=\beta_{0 \mathrm{i}}+\beta_{1 \mathrm{it}}(\text { Mon })+\beta_{2 \mathrm{it}}(\text { Wed })+\beta_{3 \mathrm{it}}(\text { Thurs })+\beta_{4 \mathrm{it}}(\text { Fri })+\beta_{5 \mathrm{it}}(\text { Sat })+\beta_{6 \mathrm{it}} \\
(\text { Sun })+\beta_{7 \mathrm{it}}(\text { NEGEVENT })+\mathrm{e}_{\mathrm{it}} \\
\beta_{0 \mathrm{i}}=\gamma_{00}+\gamma_{01}(\mathrm{SSE})+\mathrm{u}_{0 \mathrm{i}} \\
\beta_{7 \mathrm{i}}=\gamma_{10}+\gamma_{11}(\mathrm{SSE})+\mathrm{u}_{0 \mathrm{i}}
\end{gathered}
$$

In this equation, $\beta_{0 \mathrm{i}}$ is the random intercept of drinking. The level 1 intercepts and slopes are thereby a function of the level 2 between-person moderator of social selfefficacy. As seen in Table 8, the results indicated a non-significant moderating relationship $\left(\gamma_{11}=-.06, p=.21\right)$. That is, social self-efficacy did not moderate the negative social events-drinking with others relationship.

\section{Summary}

The purpose of this dissertation was to investigate multiple within- and between-person predictors of drinking with others among a Japanese college student sample. As discussed, drinking with others is an important social context within Japanese culture (Shimizu, 1990, 2000), and alcohol consumption is high (e.g. Higushi et al., 1996). Thus, it is important to identify possible antecedents of drinking. This study investigated negative social events, alcohol outcome expectancy desirability, and two types of self-efficacy as predictors of drinking with others. Hypothesis testing confirmed that these variables were significant predictors of drinking with others. The two types of self-efficacy, drinking refusal self-efficacy and social self-efficacy, were also investigated as cross-level moderators of the negative social event-drinking with other relationship. Hypothesis testing did not confirm the moderating relationship. 
Finally, building on the alcohol outcome expectancy literature, negative social events were investigated as a predictor of alcohol outcome expectancy desirability, but a significant relationship was not found. A summary table of the hypotheses and research questions can be found in Table 9.

First, I investigated negative social events as a predictor of subsequent drinking with others. This hypothesis was supported, indicating that individuals drink more with others on days that they experience increase in negative social events. This finding is consistent with existing research indicating the significance of negative social events in the lives of the Japanese. The results also indicate that drinking with others may be a way to cope with the experience of negative social events.

In contrast to the significant relationship found between negative social events and drinking with others, negative social events did not significantly predict increases in alcohol outcome expectancy desirability. That is, Hypothesis 2 was that alcohol outcome expectancy desirability would be higher on days when individuals experienced increases in negative social events. This relationship was not supported. Despite a lack of support for the relationship between negative social events and alcohol outcome expectancy desirability, alcohol outcome expectancy desirability did significantly predict drinking with others. Hypothesis 3 investigated the relationship between alcohol outcome expectancy desirability and drinking with others. Specifically, it was found that drinking with others was higher on days when individuals reported increase in alcohol outcome expectancy desirability. 
After investigating the within-person predictors of drinking with others, I turned to the investigation of two types of social self-efficacy. Specifically, I was interested in determining whether or not drinking refusal self-efficacy and social selfefficacy significantly predicted drinking with others. As hypothesized, drinking refusal self-efficacy significantly and negatively predicted drinking with others. That is, individuals with higher drinking refusal self-efficacy drank less than individuals with lower drinking refusal self-efficacy. I also investigated social self-efficacy as a predictor of drinking with others. Specifically, Research Question 1 asked whether social self-efficacy significantly predicted drinking with others. A significant and positive relationship was found, where higher social self-efficacy was associated with higher levels of drinking with others, compared to lower social self-efficacy.

Given the significant findings between negative social events and drinking with others, and drinking refusal self-efficacy and social self-efficacy and drinking with others, next, I tested drinking refusal self-efficacy and social self-efficacy as cross-level moderators of the negative social event-drinking with others relationship. The results indicated that neither drinking refusal self-efficacy nor social self-efficacy moderated the negative social events-drinking with others relationship.

In conclusion, Hypotheses 1, 3, 4 were supported; Hypotheses 2 and 5 were not. Results indicated that the direction of the relationship examined in Research Question 1 was significant and negative. The relationship examined in Research Question 2 was not supported. These results indicate that the hypothesized predictors of drinking with others were all confirmed. Negative social events, alcohol outcome 
expectancy desirability, social self-efficacy, and drinking refusal self-efficacy are important antecedents of drinking with others for Japanese college students. In the following chapter I discuss the findings in light of relevant theory. I also discuss limitations and potential implications. 


\section{CHAPTER X}

\section{Discussion}

Despite literature documenting relatively high levels of alcohol consumption among Japanese individuals, specifically in social contexts (e.g., Higuchi et al., 2006), few studies have investigated the proximal predictors of alcohol consumption among this population. Moreover, compared to individuals in relatively more independent cultures, Japanese individuals are embedded within a social system (e.g. Fiske, 1992; Kitayama \& Uchida, 2004), where maintaining social relationships is considered one of the central goals. In response to the occurrence of negative social interactions, Japanese individuals are likely to seek out individuals within their network, in order to make amends (Mascolo et al., 2003). Drinking with others may be one opportunity Japanese individuals have to make amends with their social network. The purpose of this dissertation was to investigate four possible predictors of alcohol use among Japanese college students. Specifically, this dissertation investigated negative social events, alcohol outcome expectancy desirability, drinking refusal self-efficacy and social self-efficacy as important predictors in the decision to drink alcohol with others. As hypothesized, each of these variables significantly predicted drinking with others. This dissertation also endeavored to develop drinking refusal self-efficacy and social self-efficacy as two possible moderators of the relationship between negative social events and drinking with others, although support for this relationship was not found. In this chapter, I discuss the findings in relation to the existing literature. I also provide limitations and implications of this work. 
Investigating the relationship between negative social events, alcohol outcome expectancy desirability, and drinking with others, it was necessary to capture these variables as they unfolded. In order to establish the temporal precedence implied by these relationships, I used web-based interview data that was collected from 57 Japanese college students participating in a study on cross-cultural adjustment and health. As previously discussed, daily interview data provides researchers the ability to create a sequence of events, by querying participants daily about events that occurred that day (e.g. Reis \& Gable, 2000). Capturing events, such as negative social interactions and drinking, as they occur throughout an individual's day reduces the amount of retrospective bias that is commonly found when investigating events that are highly influenced by meaning-making or difficulty recalling the sequence of events (e.g. Carney et al., 1998). Thus, this dissertation followed methodological recommendations for capturing daily interpersonal events and alcohol consumption, thereby reducing measurement error.

In the following sections I review the results of Hypotheses 1-5 and Research Questions 1 and 2. First, I discuss the experience of negative social events and their relationship to drinking with others (Hypothesis 1). Then I will discuss alcohol outcome expectancy desirability and the relationship between AOED and negative social events and drinking with others (Hypotheses 2 and 3). Finally, I will discuss self-efficacy, specifically drinking refusal self-efficacy and social self-efficacy, and their relationship to drinking with others (Hypotheses 4 and 5, and Research Questions 1 and 2). Following the discussion of the hypothesis testing, I provide strengths and 
limitations of this dissertation, as well as provide implications and potential areas of future research.

Negative Social Events and Drinking with Others

Culture is a foundational element of the self and thus influences the way the self interacts with and interprets one's environment and one's relationships with others (Triandis, 1989). Researchers have distinguished two broad categories of the cultural self: independent and interdependent (Triandis, 1989). Experiencing oneself as separate from others is more characteristic of the independent self, whereas experiencing oneself as embedded within an interrelated social network is more characteristic of the interdependent self. Within cultures that are characterized as relatively more interdependent, individuals strive to belong and to maintain social harmony (Triandis, 1989). Adhering to social norms and customs, and honoring one's social group are of utmost importance to the interdependent self.

Failure to maintain social relationships or failing to meet one's social expectations is particularly distressing for the interdependent self and, as a result, causes shame (e.g. Benedict, 1946). When individuals experience shame, they seek out others in order to make amends (Markus \& Kitayama, 1991; Mesquita \& Karasawa, 2002). Similarly, after experiencing socially isolating events, such as a disagreement within one's social group, the interdependent self seeks to conform to one's behavior to match those of the group around him/her (e.g., Markus, Kitayama, \& Heiman, 1997; Oishi \& Diener, 2001). 
The interdependent model is more prevalent in cultures such as Japan (Benedict, 1946; Markus \& Kitayama, 1991). Indeed, within Japanese culture, a giri is an important facilitator of social relationships. As previously discussed, giri is a set of social mores that encourage Japanese individuals to maintain social relationships and adhere to social norms. When an individual goes against giri, the individual strives to make amends for the transgression, and seeks out others to do so (Davies \& Ikeno, 2002).

One context where individuals may attempt to make amends is in drinking with others in their social group. Indeed, drinking with others is highly prevalent in Japan (e.g. Milne, 2003; Higuchi et al., 2006; Kitano et al., 1992) and is an opportunity to socially engage with others (Shimizu, 1990, 2000). Despite the theoretical justification for such a relationship, this association has not previously been studied.

The purpose of this dissertation was to examine the relationship between negative social events and drinking with others. Indeed, my results found support for the positive relationship between the experience of negative social events and subsequent drinking with others. By centering the negative social events variable, I am able to ascertain that on days when individuals experience more negative social events, compared to their average number of negative social events, individuals drank more with others. Thus, this result is consistent with the hypothesis that Japanese college students use drinking with others as a social context to make amends for previous disagreements or failure to maintain social relationships. 


\section{Alcohol Outcome Expectancy Desirability}

A second purpose of this dissertation was to investigate the relationship between alcohol outcome expectancy desirability, negative social events, and drinking with others. That is, it was posited that alcohol outcome expectancy desirability plays a crucial role in alcohol consumption. Indeed, the decision to drink is multifaceted, with a myriad of antecedents of drinking. Cox and Klinger (1988) outline motivational pathways in an individual's decision to drink. Specifically, Cox and Klinger (1988) posit that individuals drink in order to pursue an incentive, such as tension-reduction or social enhancement. As previously discussed, Cox and Klinger (1988) describe these motivations as the final pathway in the decision to drink (Figure 1).

In order to pursue certain motivations, individuals must have underlying beliefs about the outcomes of drinking. For example, if an individual chooses to drink in order to reduce stress, the individual must first believe that consuming alcohol has tension-reduction properties. Researchers have defined this concept as alcohol outcome expectancies, or the physiological, cognitive and behavioral outcomes of drinking (MacCorquodale \& Meehl, 1953; Del Boca et al., 2002). Alcohol outcome expectancies include positive outcomes, such as feeling relaxed or feeling more socially outgoing, and negative outcomes, such as experiencing a hangover or becoming sick from alcohol (e.g. Brown et al., 1987). Positive outcome expectancies have been shown to predict quantity (Carey, 1995), frequency (Fromme et al., 1993), and initiation and maintenance of alcohol consumption (Brown et al., 1987). 
Consistent with other measures of attitudes, which posit that an attitude is a combination of the belief about an outcome and the desirability of the outcome (e.g. Fishbein \& Azen, 1975), Armeli et al. (2005) posited that capturing the desirability of such alcohol outcomes was important. Indeed, Armeli et al. (2005) found support for multiple measurements of desirability of alcohol outcome expectancies, as daily fluctuations in desirability occurs. Daily desirability of alcohol outcome expectancies positively and significantly predicted subsequent drinking (Armeli et al., 2005). Despite the support for daily measurements of alcohol outcome expectancy desirability and the support for the predictability of desirability, no studies have furthered this body of research to other cultures or samples outside of U.S. college student samples.

The current study was the first to measure daily desirability in alcohol outcome expectancies among a Japanese college student sample. The results were consistent with the results found by Armeli et al. (2005). That is, on days when individuals experienced increases in the desirability of alcohol outcome expectancies, compared to their average level of desirability, individuals subsequently consumed more alcohol with others. Thus, alcohol outcome expectancy desirability is a useful measure of antecedents of drinking among Japanese college students.

Although increases in desirability predicted subsequent increases in drinking with others, no support was found for the relationship between negative social events and increases in desirability. Specifically, the current study investigated negative social events as a predictor of alcohol outcome expectancy desirability. This 
hypothesis was not supported, although the coefficient was in the hypothesized direction and a significant correlation between the two variables was found. Thus, a positive relationship exists between negative social events and alcohol outcome expectancies, where people who have more negative social events also have higher AOED. Future research should investigate other possible predictors of increases in alcohol outcome expectancy desirability. Further, future research should investigate alcohol outcome expectancies among a Japanese college student sample. It is possible that there are cultural differences in the expected outcomes of drinking alcohol, or that there are different alcohol expectancies related to increases in negative social events that were not captured on the checklist included in the daily interview in this study. Self-Efficacy

This dissertation investigated multiple predictors of drinking with others. Negative social events and alcohol outcome expectancy desirability were investigated as within-person predictors of alcohol use and were found to positively and significantly predict use. In addition to daily predictors of use, two types of selfefficacy, drinking refusal self-efficacy and social self-efficacy were investigated as between-person predictors of alcohol use. As predicted, both task-specific types of self-efficacy significantly predicted drinking with others.

Self-efficacy is an individual's perception of their ability to achieve a certain goal or to behave in a certain way (Bandura, 2004). Self-efficacy differs from an individual's actual ability, as it is their perception, which may be influenced by a variety of factors. Self-efficacy has been widely studied and has been shown to predict 
such as work performance, health behaviors and academic achievement (Bandura \& Locke, 2003). In the health psychology literature, self-efficacy has been posited as an important predictor of behavior (Ajzen, 1991; Stern et al., 1999). As a result of its power to predict health behavior, self-efficacy has been identified as a central factor for prevention and intervention materials. Prevention and interventions focus on increasing an individual's self-efficacy in order to increase healthy behavior (e.g. Bryan et al., 1996; Bandura, 1992, 1986).

Bandura (1992) distinguished between general self-efficacy and task-specific types of self-efficacy. That is, if a specific behavior is to be predicted, an equally specific measure of self-efficacy should be used (Salovey et al., 1998). Following the guidelines for use of task-specific self-efficacy, drinking refusal self-efficacy was chosen because of its relationship with drinking. Social self-efficacy was examined because of its potential relationship to negative social events and possible cultural ties to drinking with others (given the significance of drinking with others as a social mechanism in Japan).

Drinking refusal self-efficacy is the perceived ability to limit or decline alcohol use in certain drinking contexts (Baldwin et al., 1993; Lee \& Oei, 1993; Oei \& Burrow, 2000; Young et al., 2006). Drinking refusal self-efficacy has been welldocumented as a protective factor for alcohol use. Specifically, individuals with higher drinking refusal self-efficacy report lower drinking levels and fewer drinking episodes, compared to individuals with lower drinking refusal self-efficacy (Oei \& young, 1993; Young et al., 2006; Lee \& Oei, 1993). Consistent with previous research, in this study 
drinking refusal self-efficacy negatively and significantly predicted drinking with others. This finding extends the drinking refusal self-efficacy literature in multiple ways. First, previous studies of drinking refusal self-efficacy have utilized crosssectional or one-time self-reports, and have not investigated drinking with others as a context-specific type of alcohol use. Second, drinking refusal self-efficacy, as it predicts drinking with others, has not been investigated among a Japanese sample. Both of these contributions add to the evidence that drinking refusal self-efficacy has predictive validity in predicting alcohol consumption.

This study also investigated social self-efficacy as a possible predictor of drinking with others. Social self-efficacy is an individual's belief about their ability to engage in social tasks to either initiate or maintain relationships (Smith \& Betz, 2000). Research has shown that individuals with high social self-efficacy feel more confident addressing interpersonal problems and utilize more effective strategies to make amends, compared to individuals with lower social self-efficacy (Bilgin \& Akkapulu, 2007). Given the significance within Japanese culture to maintain social relationships and quickly make amends for negative social interactions, social self-efficacy was identified in the current study as a key factor in predicting alcohol use. Additionally, as drinking with others is seen as an important social activity and a way to interact with others (Shimizu, 1990, 2000), one purpose of this dissertation was to identify the relationship between social self-efficacy and drinking with others. In contrast to drinking refusal self-efficacy, which had an inverse relationship with drinking with others, social self-efficacy positively and significantly predicted drinking with others. 
That is, individuals with higher social self-efficacy also reported higher levels of alcohol use, compared to individuals with lower social self-efficacy. This finding lends support to the social self-efficacy literature and also further identifies drinking with others as an important behavior within Japanese culture. Furthermore, this finding strengthens the link between social motives and drinking (e.g. Cooper, 1994).

The prevalence of self-efficacy within health models and as a prevention and intervention tool suggests that self-efficacy may act as a buffer in the negative social event-drinking with others relationship. Drinking refusal self-efficacy and social selfefficacy were investigated as possible moderators of the negative social event-drinking with others relationship. It was hypothesized that drinking refusal self-efficacy would moderate the relationship such that the relationship between negative social events and drinking with others would be weaker for individuals with higher drinking refusal selfefficacy, compared to individuals with lower drinking refusal self-efficacy. This hypothesis was not supported, although a marginal relationship was found.

There are several possible explanations for this non-significant finding. First, it is possible that there was not enough power to detect this relationship. Although this study included multiple daily measurements of negative social events and drinking with others, there was only one measurement of drinking refusal self-efficacy and the total number of participants was low. As marginal significance was found, future studies with larger samples should investigate this relationship. An alternative explanation is that the drinking refusal self-efficacy and drinking with others relationships are complicated. As evidenced by the significant social self-efficacy 
findings, drinking with others is an important social interaction within Japanese culture. Additionally, the significant finding of negative social events predicting increased drinking with others also is consistent with the hypothesis that Japanese college students drink with others in an effort to make amends for prior social mishaps. Thus, if the cultural model dictates that drinking with others is beneficial to relationship well-being, drinking refusal self-efficacy may play a lesser role in predicting alcohol use. The direction of the correlation between social self-efficacy and drinking refusal self-efficacy ( $r=-.13)$ lends support for this alternative explanation, as it suggests that individuals with higher drinking refusal self-efficacy have lower social self-efficacy, compared to individuals with lower drinking refusal self-efficacy. Future studies should investigate drinking refusal self-efficacy more frequently, possibly daily, in order to better understand this complex relationship.

Social self-efficacy also was investigated as a moderator of the negative social events-drinking with others relationship. As social self-efficacy had not previously been investigated among this population or as a predictor of alcohol use, a directional relationship was not hypothesized. Support for social self-efficacy as a moderator of the negative social events-drinking with others relationship was not found. Similar to the complex relationship between drinking refusal self-efficacy, negative social events, and drinking with others, social self-efficacy may also have a complex relationship with negative social events and drinking with others. Specifically, individuals with higher social self-efficacy may be more critical of their relationships with others, or may be more sensitive to negative interactions. Indeed, self-criticism is 
an important characteristic within Japanese culture (Kitayama \&Markus, 1999). That is, Japanese college students are more likely to self-criticize, instead of self-enhance, compared to U.S. college students (Kitayama \& Markus, 1999). Indeed, in a recent meta-analytical review of cultural differences in self-serving bias, results indicated that mean self-serving bias within Japanese culture was zero, which was significantly lower than cultures such as the U.S. (Mezulis, Abramson, Hyde, \& Hankin, 2004). This results supports the hypothesis that Japanese individuals are more likely to selfcriticize, compared to Americans. Also, within social relationships, it is important to be critical of oneself, and evaluate one's role in social interactions (Benedict, 1946). Thus, individuals with higher social self-efficacy may report negative social events with more frequency, as they critically evaluated their social relationships. Indeed, a significant and positive correlation was found between negative social events and social self-efficacy $(r=.39, p<.01)$. Thus, individuals with relatively lower social selfefficacy, compared to those with higher social self-efficacy, may have less opportunities to drink with others to make amends for negative social events, as they may be less sensitive to the perception that a negative social event has occurred. In sum, this relationship is complicated. More in-depth research is necessary in order to identify the roles and relationships negative social events and social self-efficacy have with drinking with others within a Japanese sample. Future research should continue to investigate this complex relationship by more frequently measuring social selfefficacy, and possible fluctuations in social self-efficacy following the occurrence of negative social events. Similarly, other methodology may be appropriate for 
investigating this relationship. Interviews with Japanese college students regarding the occurrence of negative social events and the coping process may help shed light on this relationship.

\section{Limitations}

This dissertation is the first of its kind to investigate Japanese college student drinking at a daily level. Specifically, the results of this dissertation provide meaningful additions to the cultural psychology and alcohol use literature, as the results delineate multiple pathways to alcohol consumption for a sample where relatively no research on proximal predictors of alcohol use exists. Despite the potential implications and the use of a rigorous methodology, limitations exist.

One limitation is the large percentage of women in the current sample. The sample used in this study is a convenience sample. Future studies should attempt to recruit a more diverse sample of undergraduates, or focus solely on the drinking behaviors of Japanese women, as there may be unique characteristics of drinking behavior relative to gender, such as role conflict. Further, the relatively small sample size is a limitation of the current study. Particularly given the low occurrence of negative social events, a study including more participants, queried either more frequently or over a longer of period of time is warranted. The non-significant findings for the relationship between negative social events and alcohol outcome expectancy desirability, the marginally significant findings of the cross-level moderating effect of drinking refusal self-efficacy on negative social events and drinking with others, and the non-significant finding for the moderating effect of social self-efficacy on drinking 
with others may be due to a lack of power and the low occurrence of negative social events.

Further, although there was a significant relationship between negative social events and drinking with others, given the low occurrence of negative social events, there may be other antecedents of drinking that were not measured in the current study. Given the lack of empirical research investigating the proximal antecedents of alcohol consumption among Japanese individuals, future research should utilize qualitative methods to understand more about how and why Japanese individuals engage in drinking with others. Specifically, interviews or focus groups may be useful in querying individuals in the methods used for making amends with others and for passively declining drinking in social settings. Participant observation would also be helpful in describing the social drinking context and unique cultural drinking rituals that may occur within such a context. Results from both studies would inform future daily interview studies.

A second limitation is the amount of missing daily interview data. One source of missing data found in this study is the creation of temporal sequence between negative social events and alcohol outcome expectancy desirability and drinking with others. Specifically, because it was necessary to lag the negative social events and alcohol outcome expectancy desirability variables, this increases the amount of missing data by one set of missing data for each participant. Further, if participants did not consecutively complete the daily interviews, in addition to missing one day of data, it was impossible to create the temporal association, thus, for every one day of 
missing data, I missed three calculations. For example, if an individual did not complete Tuesday's interview (answering questions regarding negative social events and alcohol outcome expectancy desirability for Tuesday and reporting the number of drinks consumed with others on Monday night), I was unable to create the necessary temporal sequence to calculate the relationship between Monday's negative social events and alcohol outcome expectancy desirability and drinking with others on Monday night, and Tuesday's negative social events and alcohol outcome expectancy desirability and drinking with others on Tuesday night. Figure 6 demonstrates how missing one interview day affects missing data for two time periods. Additionally, the amount of missing data likely contributed to the non-significant findings for Hypothesis 2, and possibly Research Question 2. As the occurrence of negative social events was already low, missing data added to the infrequency of reports of negative social events.

Although there was missing data, the simple regressions I conducted to determine if missing data was associated with certain variables were non-significant. The non-significant results indicate that the missing data was missing at random, and was not related to participants intentionally not reporting certain activities. Thus, it is likely that on some days participants simply forgot to answer the survey, or were not near a computer and thus unable to complete the survey. One way to enhance future data collection and potentially diminish the amount of missing data would be to create a daily interview that participants could complete using their cell phone or other portable electronic device. At the time of the current study, this technology was not 
available. Future studies may also consider having a "check-in" meeting with participants throughout the 30-day study. This meeting could serve to answer any questions participants might have and to trouble-shoot problems the participants may have encountered.

A third limitation is the reliance on once-daily reports of negative social events and alcohol outcome expectancy desirabilities. Although the daily diary method is an improvement over one-time self-reports (e.g., Tennen et al., 2000), it is possible that there were fluctuations within day that may have occurred with these variables. As this is the first study to look at this relationship for a Japanese sample, future studies may want to include multiple measurements per day.

A possible fourth limitation is the use of the Drinking Refusal Self-Efficacy Questionnaire (DRSEQ; Oei et al., 2005). Although the Drinking Refusal SelfEfficacy Questionnaire-Revised (Oei et al., 2005) is the most widely used task-specific measure of self-efficacy related to alcohol consumption among U.S. samples, it has not been previously validated among a Japanese sample. Oei et al. (2005) found that this measure had concurrent and predictive validity of alcohol consumption, and recent studies have found validity for the scale among broadly defined Asian samples (i.e., Malaysian, Indonesian participants; AlMarri, Oei, \& AbRahman, 2009). The Asian participants included in the AlMarri et al. (2009) differ from Japanese drinkers because the participants in AlMarri et al. (2009) were predominantly Muslim, thus drinking was not as socially acceptable for these participants, as it is in Japan. Further, studies that have utilized the DRSEQ report very high ranges of internal consistency 
among the subscales (ranging from .85-.95; e.g., AlMarri et al., 2009, Oei et al., 2005; Christensen, Vik, \& Jarckow, 2002). These high levels of internal consistency between the subscales may indicate one common factor, as was found in this study. This suggests that further research may be necessary to determine the exact facets of drinking that this scale measures. Indeed, no studies have examined the face validity of the scale, and it may be measuring more about drinking in specific contexts, or in response to certain moods, than an individuals' actual perceived ability to refuse drinks, as the name suggests. Future studies should include testing of the face validity of the measure and use qualitative methods to examine specific behaviors used to decline drinks.

Finally, a fifth limitation is that drinking refusal self-efficacy and social selfefficacy were only measured one time throughout the study (i.e., during the initial assessment). Bandura (1997, 1986) suggests that self-efficacy fluctuates based on contextual influences, affect, and experience. It is possible that there were fluctuations in the task-specific self-efficacy that were not captured by the single measurement. For example, drinking refusal self-efficacy may have been higher in certain contexts compared to others. Further, given the daily fluctuations in negative social interactions, social self-efficacy may likely fluctuate as well. Future studies should incorporate daily measurements of self-efficacy in an attempt to capture fluctuations.

\section{Theoretical and Practical Implications}

Despite some limitations, the current study makes several notable contributions to the field, as it confirmed negative social events, alcohol outcome expectancy 
desirability, drinking refusal self-efficacy, and social self-efficacy as predictors of drinking with others. Additionally, the meaningful contributions of this work include identifying within-person variation in drinking among Japanese college students. This work also provides a thorough description of negatively-appraised social events. This is a significant contribution, as the current study investigated the appraisals of events, thus reducing researcher bias in regards to what was considered a negative compared to a positive event.

The significant relationship between the experience of negative social events and subsequent drinking has implications for efforts to reduce alcohol consumption. In particular, for universities, where there are high levels of alcohol consumption (Baer, 2002), the significant results of this study indicate that drinking with others serves a purpose in helping Japanese college student maintain social relationships, which is an important goal of the relatively more interdependent self. Moreover, the results of this study provide support for tailoring interventions to address the unique cultural antecedents of drinking. Specifically, the evidence that individuals in this study drank with others in response to negative interpersonal events differs from U.S. models that show increases in solitary drinking on days with higher negative interpersonal events among college students (Mohr et al., 2005).

Thus, interventions that simply seek to eliminate opportunities where students can engage in drinking with others may be limited in their scope, and have potential negative effects on student's social relationships. In conjunction with the negative significant relationship found between drinking refusal self-efficacy and drinking with 
others, college student drinking interventions may want to focus on providing contexts where alcohol plays a secondary role in the activities, or where students feel able to drink less. Future studies should investigate other methods Japanese college students utilize to maintain social relationships and to make amends.

\section{Conclusion}

This study provides a unique investigation into the cultural, contextual, and individual components of alcohol consumption. Evidence of a relationship between the experience of negative social events and subsequent drinking was found. Alcohol outcome expectancy desirability also predicted subsequent drinking. Moreover, drinking refusal self-efficacy was a significant protective factor for drinking with others and marginal support of drinking refusal self-efficacy as a buffer in the negative social event and drinking with others relationship was found. Further, this study was the first to document social self-efficacy as a positive and significant predictor of drinking with others. Despite some possible limitations, the present study makes several significant contributions to the alcohol and cultural literature. Among the strengths of this study is the use of daily process methodology to examine drinking behavior among a Japanese college-student sample. Specifically, to my knowledge, this is the first study to examine day-to-day variations in alcohol consumption and proximal predictors of alcohol consumption among a Japanese sample. This data provides a more thorough description of alcohol use among Japanese college students, beyond simple self-reports of drinking behavior. 
Moreover, this study was the first to investigate daily fluctuations in the desirability of alcohol outcome expectancies among a Japanese sample. The significant relationship found between increases in daily desirability and drinking with others supports current research that has documented the value of desirability of alcohol outcome expectancies in the prediction of daily alcohol use (Armeli et al., 2005). 


\section{Endnotes}

${ }^{1}$ It should be noted that although descriptions provide many important differences that may exist within cultural systems, however, these distinctions must be regarded as general tendencies of a group as a whole; individual variations exist (Markus \& Kitayama, 1991).

${ }^{2}$ Austria, Finland, Argentina, Germany, Norway, Japan, Hungary, the Czech Republic, France, Italy, the United Kingdom, Switzerland, Sweden, Iceland, The Netherlands, Mexico, Israel, the U.S., Costa Rica, Spain, Brazil, Sri Lanka, and Uganda were included in the analysis.

${ }^{3}$ The Miyagi prefecture is located in the northeastern region of Japan, approximately 3 hours, by car, from Tokyo, the capital of Japan. 
Figure 1. Proposed theoretical model of alcohol use.

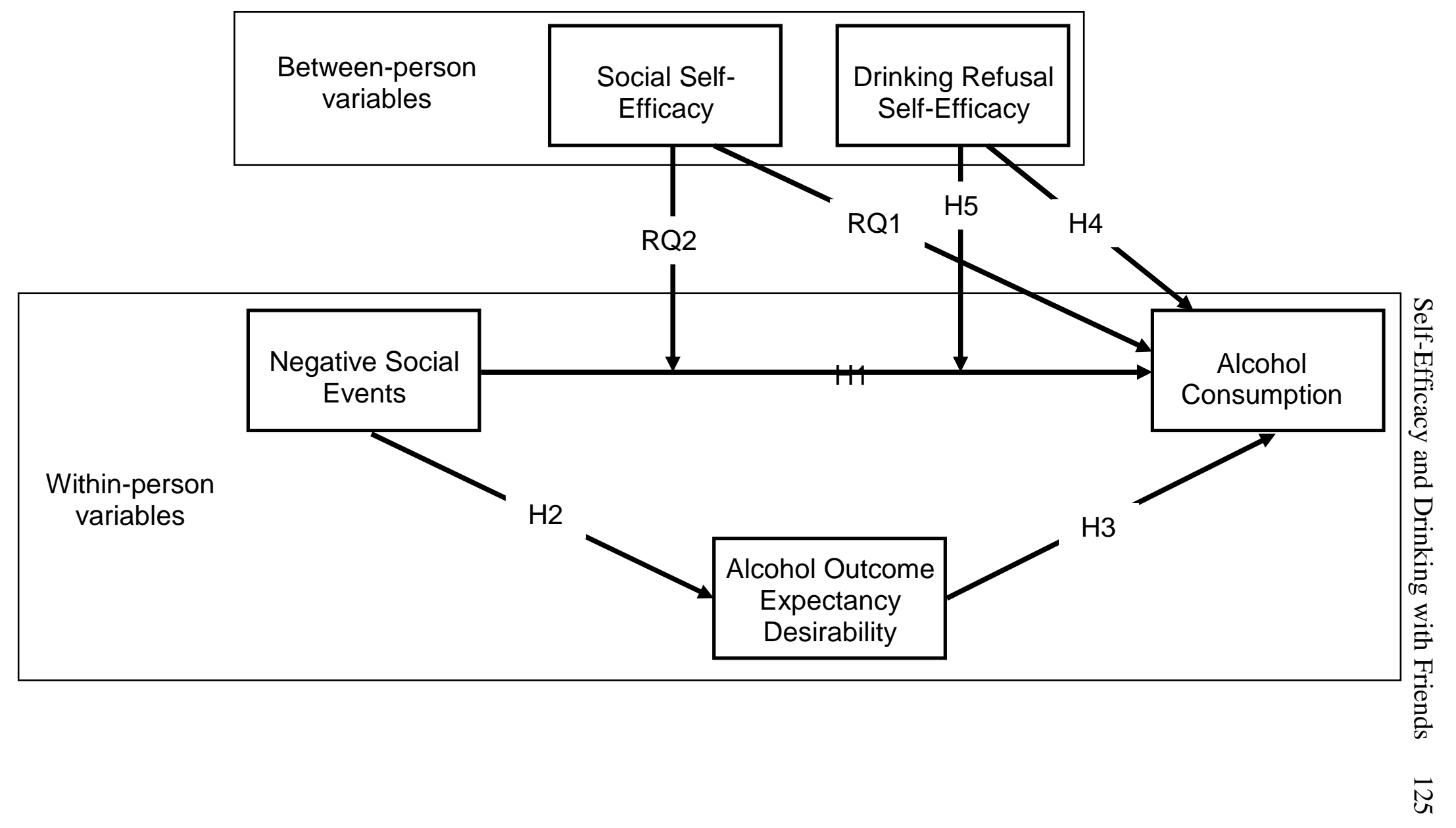


Figure 2. Predictors of alcohol consumption.

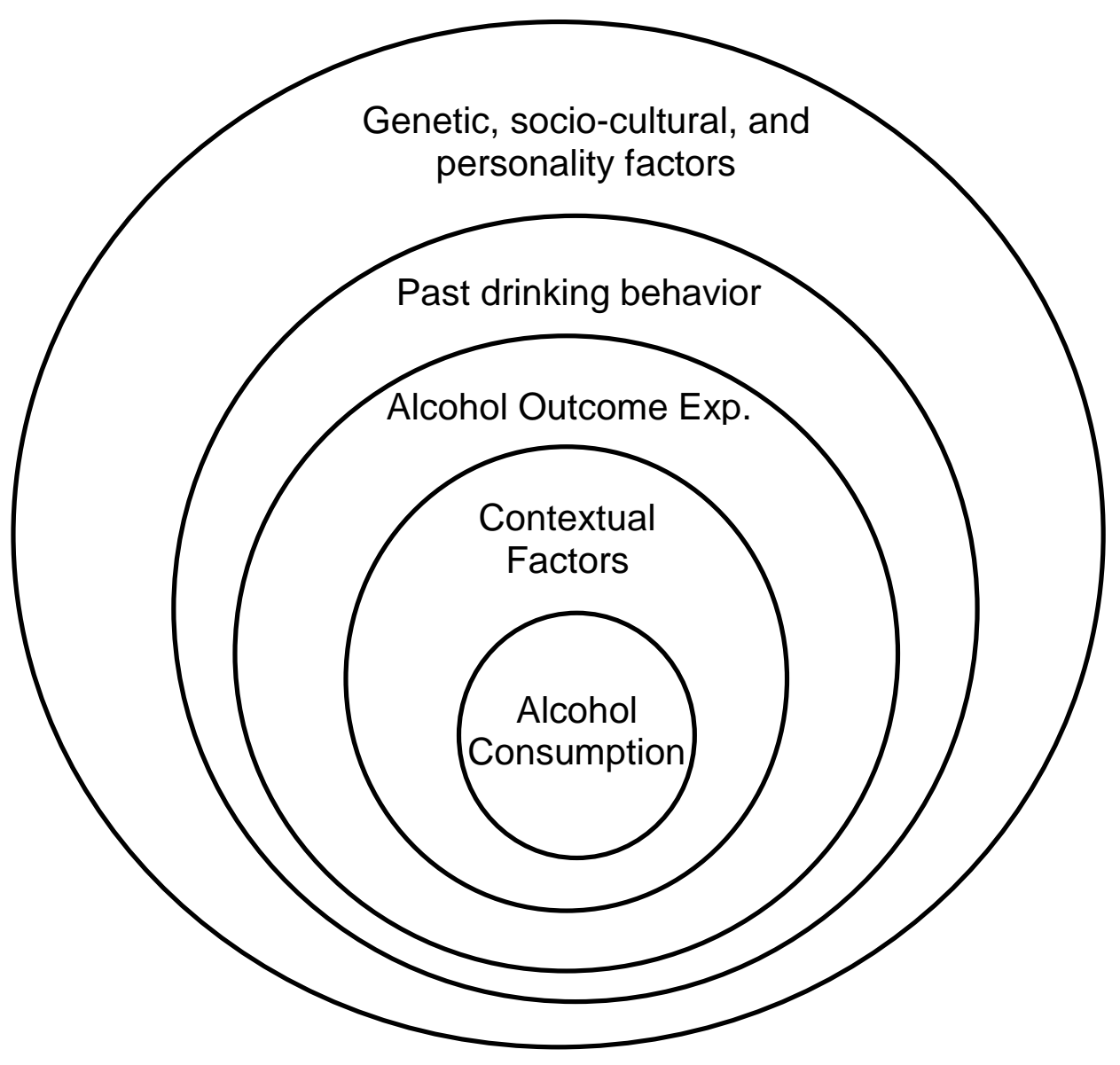


Figure 3. Transactional model of stress, drinking, and coping.

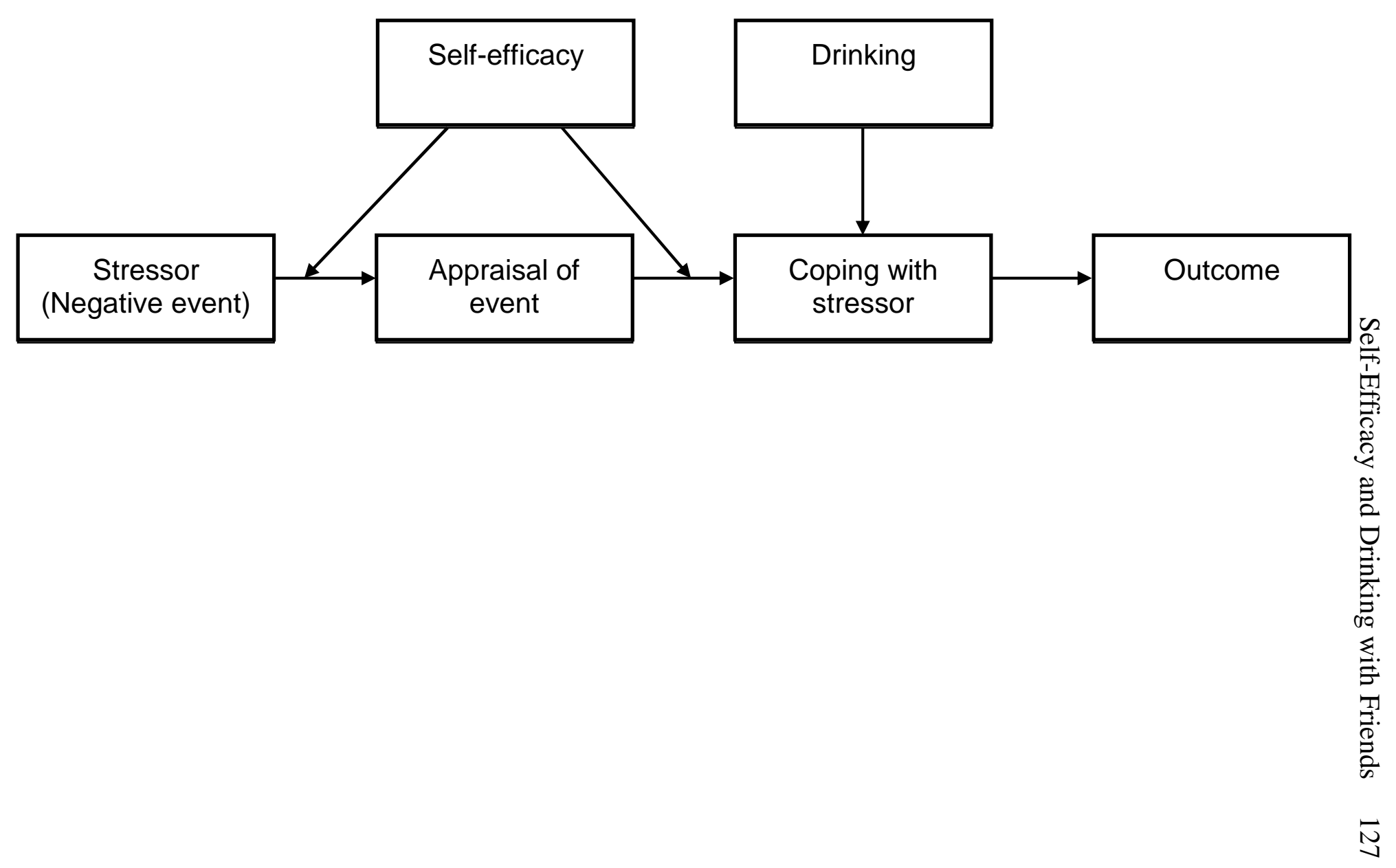


Figure 4. Key measurements.

\begin{tabular}{|l|l|}
\hline Measure & Timing \\
\hline \hline Drinking Refusal Self-Efficacy & Initial Assessment \\
\hline Social Self-Efficacy & Initial Assessment \\
\hline Daily Negative Social Events & Daily Survey (since the last interview) \\
\hline Alcohol Outcome Expectancy Desirability & Daily Survey (current) \\
\hline Daily Consumption & Daily Survey (last night) \\
\hline
\end{tabular}


Figure 5. Daily interview protocol

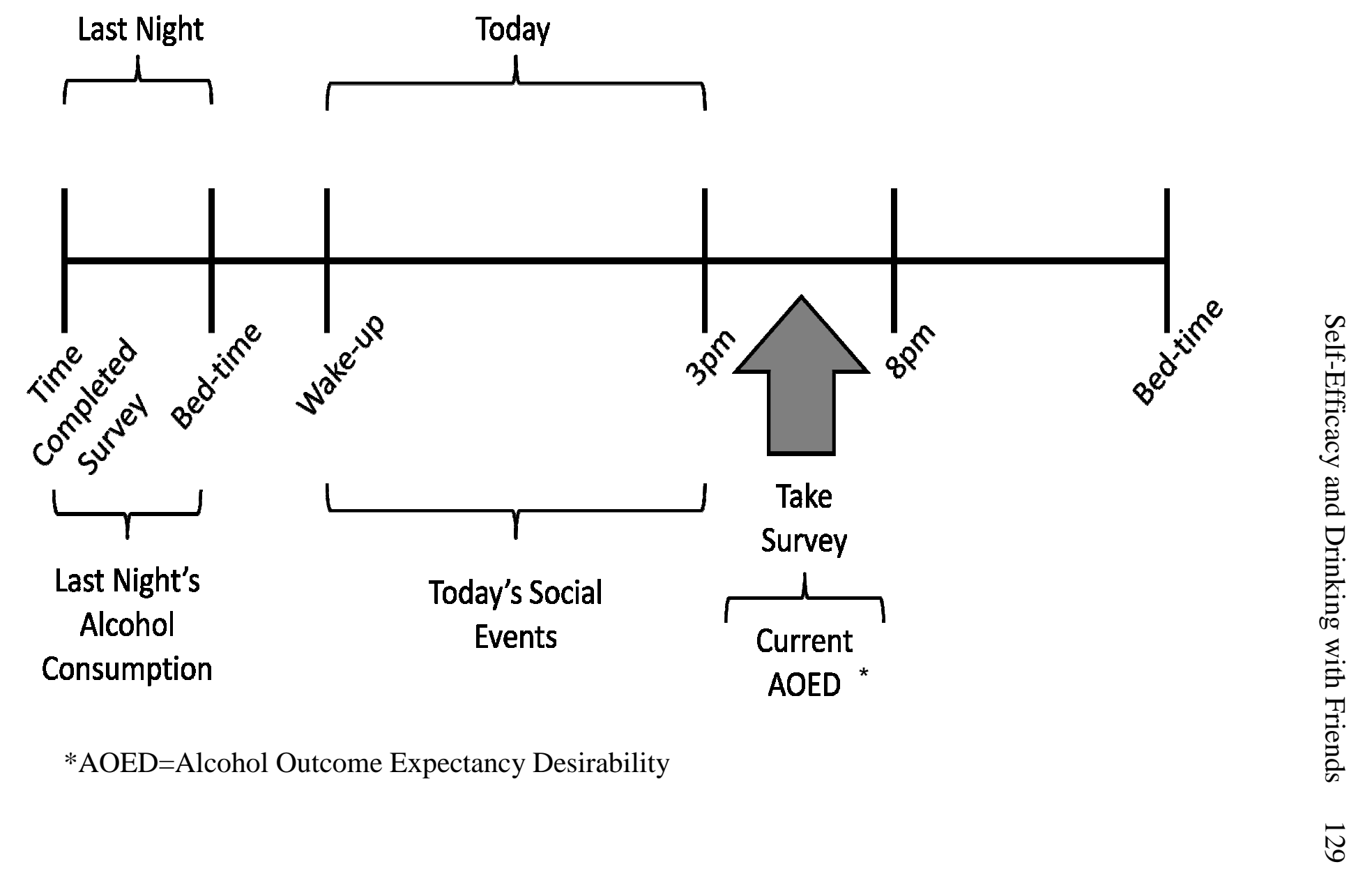


Figure 6. Example of missing data

\begin{tabular}{|c|c|c|c|}
\hline Study Day & $\begin{array}{c}\text { Today's Negative } \\
\text { Social Events } \\
\text { (As reported) }\end{array}$ & $\begin{array}{c}\text { Today's Negative } \\
\text { Social Events } \\
\text { (Lagged) }\end{array}$ & $\begin{array}{c}\text { Last Night's } \\
\text { Drinking with } \\
\text { Others }\end{array}$ \\
\hline 1 & 7 & - & 4 \\
\hline 2 & 6 & 7 & 4 \\
\hline 3 & 8 & 6 & 5 \\
\hline 4 & 1 & 8 & 0 \\
\hline 5 & missing & 1 & missing \\
\hline 6 & 5 & 5 & 4 \\
\hline 7 & 3 & 3 & missing \\
\hline 8 & missing & missing & 0 \\
\hline 9 & 1 & 2 & 1 \\
\hline 10 & 2 & & \\
\hline
\end{tabular}

Note: Shaded cells are pairs that can be used for data analysis. 


\begin{tabular}{|c|c|c|c|c|c|c|c|}
\hline \multicolumn{8}{|c|}{ Table 1. Between-person correlations } \\
\hline & $\begin{array}{c}\text { Mea } \\
\mathrm{n}\end{array}$ & SD & $\begin{array}{c}\text { Drinking With } \\
\text { Others }\end{array}$ & $\begin{array}{c}\text { Negative } \\
\text { Social Events }\end{array}$ & $\begin{array}{c}\text { Alcohol } \\
\text { Outcome } \\
\text { Expectancy } \\
\text { Desirability }\end{array}$ & $\begin{array}{l}\text { Drinking } \\
\text { Refusal Self- } \\
\text { Efficacy }\end{array}$ & $\begin{array}{c}\text { Social Self- } \\
\text { Efficacy }\end{array}$ \\
\hline Age & $\begin{array}{c}23.1 \\
2 \\
\end{array}$ & 2.96 & -.19 & .03 & -.25 & -.08 & -.11 \\
\hline Gender & & & -.24 & $-.29 *$ & $-.46^{* *}$ & .00 & -.13 \\
\hline $\begin{array}{l}\text { Internation } \\
\text { al Student }\end{array}$ & & & -.20 & .22 & $-.25^{\dagger}$ & .00 & .20 \\
\hline $\begin{array}{l}\text { Drinking } \\
\text { With } \\
\text { Others } \\
\end{array}$ & & & 1.00 & & & & \\
\hline Neg. Event & 1.27 & 2.51 & -.11 & 1.00 & & & \\
\hline AOED & 1.68 & .69 & .21 & $.30 *$ & 1.00 & & \\
\hline DRSE & 4.07 & .90 & $-.39 * *$ & .14 & -.17 & 1.00 & \\
\hline SSE & 2.82 & .77 & .22 & $.39 * *$ & .19 & -.13 & 1.00 \\
\hline
\end{tabular}

Note: Between-person correlations. Within-person variables (Drinking with Others, Negative Social Events, and Alcohol Outcome Expectancy Desirability) are aggregated across the 30 study days. Gender is coded as $0=$ male, $1=$ female. International student is coded as $0=$ student studying in Japan and $1=$ student studying in the U.S.

$\dagger p<.08$

$* p<.05$

$* * p<.01$ 


\begin{tabular}{|c|c|c|c|c|}
\hline Variable & Coefficient & SE & p-value & Confidence Interval \\
\hline Intercept & -1.16 & .45 & .01 & $.13, .78$ \\
\hline Negative Social Event & .23 & .04 & .00 & $1.16,1.37$ \\
\hline Sunday & 1.41 & .52 & .007 & $1.49,11.20$ \\
\hline Monday & .48 & .56 & .39 & $.54,4.80$ \\
\hline Wednesday & .29 & .55 & .59 & $.46,3.92$ \\
\hline Thursday & 1.30 & .46 & .005 & $1.48,9.00$ \\
\hline Friday & .28 & .50 & .57 & $.50,3.53$ \\
\hline Saturday & 1.57 & .52 & .003 & $1.73,13.34$ \\
\hline \multicolumn{5}{|l|}{ Variance Components } \\
\hline Intercept & \multicolumn{4}{|l|}{$.75 * * *$} \\
\hline Negative Social Event & \multicolumn{4}{|l|}{$.05^{*}$} \\
\hline
\end{tabular}

Note: Dependent variable is Drinking with Others

${ }^{*} p<.05$

$* * p<.01$

$* * * \mathrm{p}<.001$ 


\begin{tabular}{|l|c|c|c|}
\hline Table 3. Hypothesis 2 & Coefficient & SE & p-value \\
\hline Variable & 1.62 & .14 & .00 \\
\hline Intercept & .02 & .02 & .21 \\
\hline Negative Social Events & .18 & .15 & .25 \\
\hline Sunday & -.08 & .14 & .59 \\
\hline Monday & -.17 & .10 & .09 \\
\hline Wednesday & -.07 & .13 & .57 \\
\hline Thursday & .00 & .11 & .99 \\
\hline Friday & .17 & .16 & .28 \\
\hline Saturday & \multicolumn{5}{l}{} \\
\hline \multicolumn{5}{|l|}{} \\
\hline Variance Components & $.27^{* * *}$ \\
\hline Intercept & .05 \\
\hline Negative Social Events
\end{tabular}

Note: Dependent Variable is Alcohol Outcome Expectancy Desirability

$* p<.05$

$* * p<.01$

$* * * \mathrm{p}<.001$ 


\begin{tabular}{|l|c|c|c|c|}
\hline Table 4: Hypothesis 3 & Coefficient & SE & $\begin{array}{c}\text { p- } \\
\text { value }\end{array}$ & $\begin{array}{c}\text { Confidence } \\
\text { Interval }\end{array}$ \\
\hline Variable & -1.12 & .23 & .001 & $.21, .52$ \\
\hline Intercept & .98 & .15 & .001 & $1.98,3.56$ \\
\hline $\begin{array}{l}\text { Alcohol Outcome Expectancy } \\
\text { Desirability }\end{array}$ & 1.00 & .30 & .001 & $1.50,4.95$ \\
\hline Sunday & .26 & .34 & .76 & $.67,2.50$ \\
\hline Monday & -.04 & .01 & .001 & $.95, .97$ \\
\hline Wednesday & 1.07 & .34 & .002 & $1.49,5.71$ \\
\hline Thursday & .13 & .33 & .71 & $.59,2.17$ \\
\hline Friday & 1.07 & .35 & .003 & $1.50,5.81$ \\
\hline Saturday & & & \\
\hline & & & \\
\hline Variance Components & $1.01 * * *$ \\
\hline Intercept & $.49 * * *$ \\
\hline $\begin{array}{l}\text { Alcohol Outcome Expectancy } \\
\text { Desirability }\end{array}$ &
\end{tabular}

Note: Dependent variable is Drinking with Others $* * * \mathrm{p}<.001$ 


\begin{tabular}{|l|c|c|c|c|}
\hline Table 5. Hypothesis 4 & Coefficient & SE & $\begin{array}{c}\text { p- } \\
\text { value }\end{array}$ & $\begin{array}{c}\text { Confidence } \\
\text { Interval }\end{array}$ \\
\hline Level-1 Variable & -1.23 & .44 & .009 & $.12, .72$ \\
\hline Intercept & 1.38 & .51 & .008 & $1.45,10.83$ \\
\hline Sunday & .44 & .55 & .42 & $.53,4.52$ \\
\hline Monday & .06 & .57 & .92 & $.35,3.22$ \\
\hline Wednesday & 1.26 & .47 & .008 & $1.41,8.77$ \\
\hline Thursday & .56 & .53 & .30 & $.61,4.97$ \\
\hline Friday & 1.57 & .53 & .004 & $1.70,13.57$ \\
\hline Saturday & \multicolumn{5}{|l|}{} \\
\hline \multicolumn{5}{|l|}{} \\
\hline Level-2 Variable & -.53 & .13 & .001 & $.45, .76$ \\
\hline \multicolumn{5}{|l|}{} \\
\hline Varinking Refusal Self-Efficacy & Intercept Components & $.54 * * *$ \\
\hline
\end{tabular}

Note: Dependent variable is Drinking with Others $* * * \mathrm{p}<.001$ 


\begin{tabular}{|l|c|c|c|c|}
\hline Table 6. Hypothesis 5 & Coefficient & SE & p-value & $\begin{array}{c}\text { Confidence } \\
\text { Interval }\end{array}$ \\
\hline Level-1 Variable & -1.25 & .46 & .009 & $.11, .72$ \\
\hline Intercept & .23 & .05 & .001 & $1.13,1.41$ \\
\hline Negative Social Events & 1.40 & .53 & .009 & $1.44,11.56$ \\
\hline Sunday & .46 & .58 & .42 & $.51,4.95$ \\
\hline Monday & .31 & .57 & .59 & $.45,4.12$ \\
\hline Wednesday & 1.29 & .48 & .007 & $1.42,9.20$ \\
\hline Thursday & .26 & .52 & .61 & $.47,3.62$ \\
\hline Friday & 1.56 & .53 & .004 & $1.68,13.59$ \\
\hline Saturday & & & \\
\hline \multicolumn{5}{|l|}{} \\
\hline Level-2 Variable & -.56 & .12 & .001 & $.44, .73$ \\
\hline Drinking Refusal Self-Efficacy & -.09 & .05 & .08 & $.83,1.01$ \\
\hline $\begin{array}{l}\text { Drinking Refusal Self-Efficacy X } \\
\text { Negative Event Interaction }\end{array}$ & \multicolumn{5}{|l}{} \\
\hline \multicolumn{4}{|l|}{} \\
\hline Variance Components & $.54^{* * *}$ \\
\hline Intercept & $.05^{*}$ \\
\hline
\end{tabular}

Note: Dependent variable is Drinking with Others $* * * \mathrm{p}<.001$ 


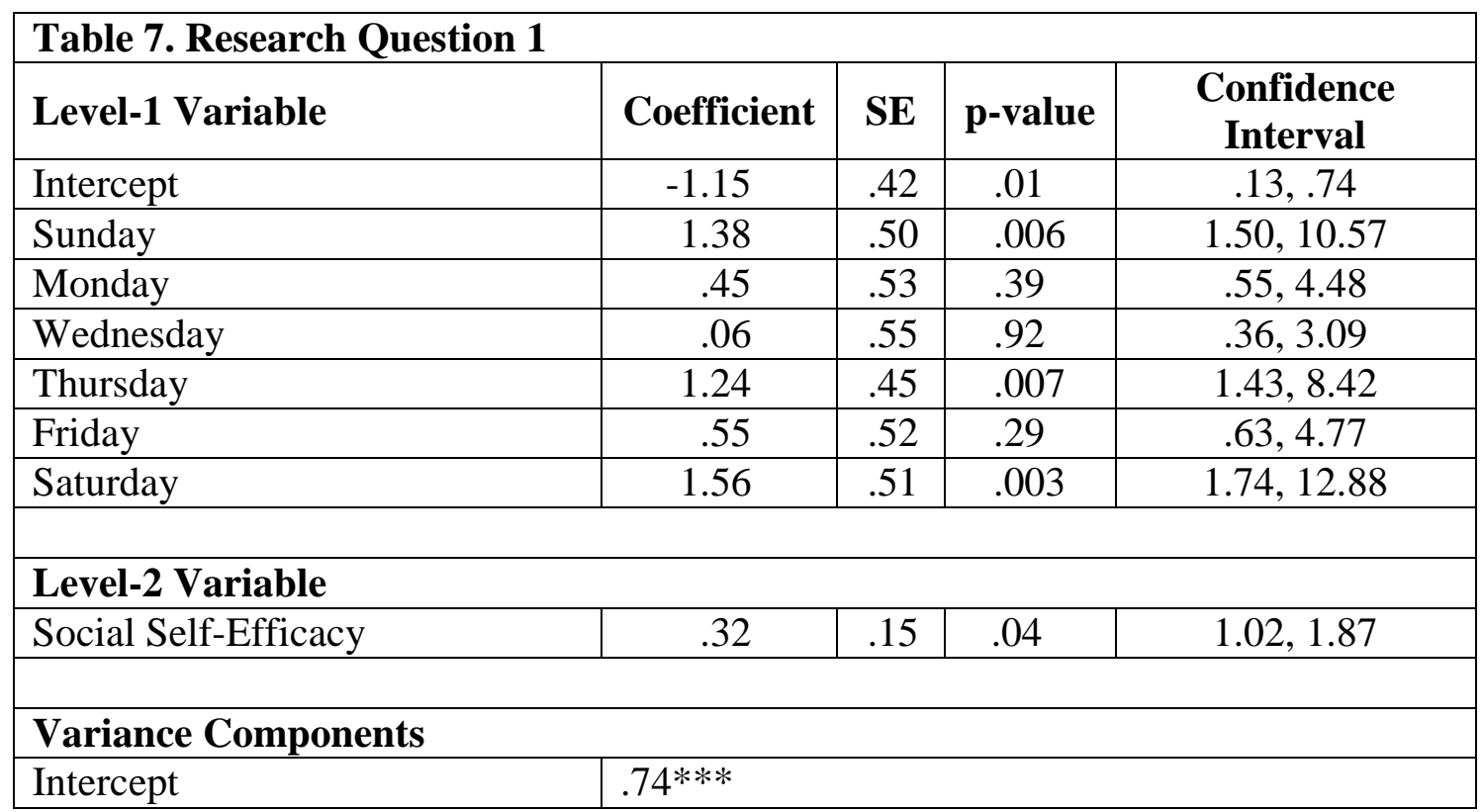

Note: Dependent variable is Drinking with Others $* * * \mathrm{p}<.001$ 
Self-Efficacy and Drinking with Friends 138

\begin{tabular}{|l|c|c|c|c|}
\hline Table 8. Research Question 2 & Coefficient & SE & p-value & $\begin{array}{c}\text { Confidence } \\
\text { Interval }\end{array}$ \\
\hline Level-1 Variable & -1.17 & .43 & .009 & $.13, .73$ \\
\hline Intercept & .23 & .03 & .001 & $1.18,1.35$ \\
\hline Negative Social Events & 1.40 & .51 & .006 & $1.51,10.95$ \\
\hline Sunday & .49 & .54 & .37 & $.57,4.71$ \\
\hline Monday & .26 & .54 & .63 & $.45,3.75$ \\
\hline Wednesday & 1.29 & .45 & .005 & $1.50,8.75$ \\
\hline Thursday & .26 & .49 & .60 & $.50,3.38$ \\
\hline Friday & 1.56 & .51 & .003 & $1.74,12.91$ \\
\hline Saturday & & & \\
\hline \multicolumn{4}{|l|}{} \\
\hline Level-2 Variable & .37 & .14 & .01 & $1.09,1.95$ \\
\hline Social Self-Efficacy & -.06 & .06 & .21 & $.86,1.03$ \\
\hline $\begin{array}{l}\text { Social Self-Efficacy X Negative } \\
\text { Events }\end{array}$ & & & \\
\hline \multicolumn{4}{|l|}{} \\
\hline Variance Components & $.76^{* * *}$ \\
\hline Intercept & $.04^{\dagger}$ \\
\hline Negative Social Events
\end{tabular}

Note: Dependent variable is Drinking with Others

$\dagger p<.08$

$* * * \mathrm{p}<.001$ 


\begin{tabular}{|c|c|c|}
\hline Hypothesis & & Supported? \\
\hline $\begin{array}{l}\text { Hypothesis } \\
\quad 1\end{array}$ & $\begin{array}{l}\text { Students will drink more with others on days } \\
\text { characterized by greater negative social events } \\
\text { compared to days with fewer negative social events. }\end{array}$ & Supported \\
\hline $\begin{array}{l}\text { Hypothesis } \\
2\end{array}$ & $\begin{array}{l}\text { The desirability of alcohol outcome expectancies } \\
\text { related to relationship building and tension reduction } \\
\text { will be higher on days with greater negative social } \\
\text { events, compared to days with fewer negative social } \\
\text { events. }\end{array}$ & $\begin{array}{c}\text { Not } \\
\text { Supported }\end{array}$ \\
\hline $\begin{array}{l}\text { Hypothesis } \\
3\end{array}$ & $\begin{array}{l}\text { Drinking with others will be higher on days when } \\
\text { alcohol outcome expectancies related to tension } \\
\text { reduction and relationship building are rated as more } \\
\text { desirable, compared to days with lower rated } \\
\text { desirability. }\end{array}$ & Supported \\
\hline $\begin{array}{l}\text { Hypothesis } \\
\quad 4\end{array}$ & $\begin{array}{l}\text { Drinking refusal self-efficacy will significantly and } \\
\text { negatively predict drinking with others. }\end{array}$ & Supported \\
\hline $\begin{array}{l}\text { Hypothesis } \\
5\end{array}$ & $\begin{array}{l}\text { Negative social events will have less of an effect on } \\
\text { drinking for individuals who have high perceived } \\
\text { drinking refusal self-efficacy, compared to those with } \\
\text { low drinking refusal self-efficacy. }\end{array}$ & $\begin{array}{c}\text { Not } \\
\text { Supported }\end{array}$ \\
\hline $\begin{array}{l}\text { Research } \\
\text { Question 1 }\end{array}$ & $\begin{array}{l}\text { What is the relationship between social self-efficacy } \\
\text { and drinking with others? }\end{array}$ & Supported \\
\hline $\begin{array}{l}\text { Research } \\
\text { Question } 2\end{array}$ & $\begin{array}{l}\text { Does social self-efficacy moderate the relationship } \\
\text { between negative social events and drinking? If so, } \\
\text { what is the direction of this relationship? }\end{array}$ & $\begin{array}{c}\text { Not } \\
\text { Supported }\end{array}$ \\
\hline
\end{tabular}


References

Abrams, D. B., \& Niara, R. S. (1987). Social learning theroy of alcohol use and abuse. In H. Blane, \& K. Leonard, Psychological theories of drinking and alcoholism (pp. 131-178). New York: Guildford Press.

Affleck, G., Zautra, A., Tennen, H., \& Armeli, S. (1999). Multilevel daily process designs for consulting and clinical psychology: A preface for the perplexed. Journal of Consulting and Clinical Psychology, 67, 746-754.

Aiken, L. S., \& West, S. G. (1991). Multiple regression: Testing and interpreting interactions. Newbury Park, CA: Sage.

Ajzen, I. (1991). The theory of planned behavior. Oganizational Behavior and Human Decision Processes, 50, 179-211.

Akechi, T., Iwasaki, M., Uchitomi, Y., \& Tsugane, S. (2006). Alcohol consumption and suicide among middle-aged men in Japan. The British Journal of Psychiatry, 188, 231-236.

Allison, A. (1994). Nightwork: Sexuality, pleasure, and corporate masculinity in a Tokyo hostess club. Chicago: University of Chicago Press.

Allison, P. D. (2002). Missing Data. Thousand Oaks, CA: Sage.

AlMarri, T.S.K., Oei, T.P.S., AbRahman, R.M. (2009). Validation of the drinking refusal self-efficacy questionaniare in Arab and Asian samples, Psychology of Addictive Behaviors, 34, 776-778. 
Argeriou, M. (1975). Daily alcohol consumption patterns in Boston: Some findings and a partial test of the Tuesday hypothesis, Journal of Studies on Alcohol, 36, 1578-1583.

Armeli, S., Mohr, C., Todd, M., Maltby, N., Tennen, H., Carney, M. A., et al. (2005). Daily evaluation of anticipated outcomes from alcohol use among college students. Journal of Social and Clinical Psychology, 24, 767-792.

Arnett, J. J. (2000). Emerging Adulthood: A theory of development from the late teens through the twenties. American Psychologist, 55, 469-480.

Babor, T. F., Hofmann, M., DelBoca, F. K., Hesselbrock, V. M., Meyer, R. E., Dolinsky, Z. S., et al. (1992). Types of alcoholics: Evidence for an empirically derived typology based on indicators of vulnerability and severity. Archives of General Psychiatry, 49, 599-608.

Baer, J. (2002). Student factors: Understanding individual variation in college drinking. Journal of Studies on Alcohol, Supplement No. 14, 40-53.

Baer, J., \& Carney, M. (1993). Biases in the perceptions of the consequences of alcohol use among college students. Journal of Studies on Alcohol, 54, 54-60.

Baldwin, A.R., Oei, T.P.S., \& Young, R. (1993). To drink or not to drink: the differential role of alcohol expectancies and drinking refusal self-efficacy in quantity and frequency of alcohol consumption, Cognitive Therapy and Research, 17, 511-530.

Bandura, A. (2004). Health promotion by social cognitive means. Health Education \& Behavior, 31, 143-164. 
Bandura, A. (1997). Self-efficacy: The exercise of control. New York: Freeman. Bandura, A. (1992). Self-efficacy mechanism in psychobiologic functioning. In R. Schwarzer, Self-efficacy: Thought control of action (pp. 355-394). Washington, D.C.: Hemisphere.

Bandura, A. (1986). Social foundations of thought and action: A social cognitive theory. Englewood Cliffs, NJ: Prentice-Hall.

Bandura, A. (1977). Social learning theory. Englewood Cliffs, NJ: Prentice Hall. Bandura, A., \& Locke, E. (2003). Negative self-efficacy and goal effects revisited. Journal of Applied Psychology, 31, 87-99.

Bandura, A., Pastorelli, C., Barbaranelli, C., \& Caprara, G. V. (1999). Self-efficacy pathways to childhood depression. Journal of Personality and Social Psychology, 75, 258-269.

Benedict, R. (1946). The chrysanthemum and the sword: Patterns of Japanese culture. Boston: Houghton-Mifflin.

Bilgin, M., \& Akkapulu, E. (2007). Some variables predicting social self-efficacy expectation. Social Behavior and Personality, 35, 777-7888.

Bloomfield, K., Allamani, A., Beck, F., Helmersson-Bergmark, K., Csemy, L., Eisenback-Stangl, I., et al. (2005). Gender, culuture, and alcohol problems: A multi-national study. Project Final Report. Institute for Medical Informatics, Biometrics, \& Epidemiology. Berlin: Charite Universitatsmedizin.

Bolger, N., Davis, A., \& Rafaeli, E. (2003). Diary methods: Capturing life as lived, Annual Review of Psychology, 54, 579-616. 
Bolger, N., DeLongis, A., Kessler, R. C., \& Schilling, E. A. (1989). Effects of daily stress on negative mood. Journal of Personality and Social Psychology , 57 (5), 808-818.

Bond, J. C., Greenfield, T. K., Roberts, S. C., \& Korcha, R. (2010, May). Alcohol consumption and alcohol-related problems: A cross-national multilevel GENACIS analysis. 36th Annual Epidemiological Symposium of the Kettil Bruun Society for Social and Epidemiological Study of Alcohol. Lausanne, Switzerland.

Borsari, B., \& Carey, K. (2001). Peer influences on college drinking: A review of the research. Journal of Substance Abuse, 13, 391-424.

Brewer, M. (2000). Research Design and Issues of Validity. In H. Reis, \& C. Judd, Handbook of Research Methods in Social and Personality Psychology. Cambridge: Cambridge University Press.

Brown, S. A., Christiansen, B. A., \& Goldman, M. S. (1987). The alcohol expectancy questionnaire: An instrument for the assessment of adolescent and adult alcohl expectancies. Journal of Studies on Alcohol, 48, 483-491.

Bryan, A.D., Aiken, L.S., \& West, S.G. (1996). Increasing condom use: Evaluation of a theory-based intervention to prevent sexually transmitted diseases in young women, Health Psychology, 15, 371-382.

Bryk, A. S., \& Raudenbush, S. W. (1992). Hierarchical linear models: Applications and data analysis methods. Newbury Park, CA: Sage. 
Campbell, J. M., \& Oei, T. (2010). The intergenerational transference of alcohol use behaviour from parent to offspring: A test of the cognitive model. Psychology of Addictive Behaviors, 35, 714-716.

Carey, K. (1995). Alcohol-related expectancies predict quantity and frequency of heavy drinking among college students. Psychology of Addictive Behaviors, 9, 236-241.

Carney, M. A., Tennen, H., Affleck, G., Del Boca, F. K., \& Kranzler, H. R. (1998). Levels and patterns of alcohol consumption using timeline follow-back, daily diaries, and real-time "electronic interviews", Journal of Studies on Alcohol, 59, 447-454.

Christiansen, M., Vik, P.W., Jarchow, A. (2002). College student heavy drinking in social contexts versus alone, Psychology of Addictive Behaviors, 27, 393-404.

Coe, C. L., \& Levine, S. (1986). Psychoimmunology: An old idea whose time has come. In P. Barchas, Social physiology of social relations. Oxford, England: Oxford University Press.

Cohen, S., \& Wills, T. A. (1985). Stress, social support, and the buffering hypothesis. Psychological Bulletin, 98, 310-357.

Cooper, M. L., Russell, M., Skinner, J. B., \& Windle, M. (1992). Developmnet and validation of a three-dimensional measure of drinking motives. Psychological Assessment, 4, 123-132.

Cooper, M. (1994). Motivations for alcohol use among adolescents: Development and validation of a four-factor model. Psychological Assessment, 6, 117-128. 
Cox, W., \& Klinger, E. (1988). A motivational model for alcohol use. Journal of Abnormal Psychology, 97, 168-180.

Csikszentmihalyi, M. (1997). Finding flow: The Psychology of Engagement with Everyday Life. New York: Basic Books.

Darkes, J., \& Goldman, M. (1993). Expectancy challenge and drinking reduction: Experimental evidence for a mediational process. Journal of Consulting and Clinical Psychology, 61, 344-353.

Darkes, J., \& Goldman, M. (1998). Expectancy challenge and drinking reduction: Process and structure in the alcohol expectancy network. Experimental and Clinical Psychopharamcology, 6, 64-76.

Davies, R. J., \& Ikeno, O. (2002). The Japanese Mind: Understanding Contemporary Japanese Culture. Boston, MA: Turtle Publishing.

Dearing, R. L., Stuewig, J., \& Tangney, J. P. (2005). On the importance of distinguishing shame from guilt: Relations to problematic alcoholic and drug use. Addictive Behaviors, 1392-1404.

DelBoca, F., Darkes, J., Goldman, M. S., \& Smith, G. T. (2002). Advancing the expectancy concept via the interplay between theory and research. Alcoholism: Clinical and Experinmental Research, 26, 926-935.

DeLongis, A., Folkman, S., \& Lazarus, R. S. (1988). The impact of daily stress on health and mood: Psychological and social resources as mediators. Journal of Personality and Social Psychology, 54, 486-495. 
DeMente, B. (1995). Japan made easy: All you need to know to enjoy Japan (2nd ed.). McGraw-Hill.

DeSivilya, H. S., \& Eizen, D. (2005). Conflict management in work teams: The role of social self-efficacy and group identification. International Journal of Conflict Management, 16, 193-208.

DeWitz, S. J., \& Walsh, W. B. (2002). Self-efficacy and college student satisfaction. Journal of Career Assessment, 10, 315-325.

Enders, C. K., \& Tofighi, D. (2007). Centering predictor variables in cross-sectional multilevel models: A new look at an old issue. Psychological Methods, 12, 121-138.

Fishbein, M., \& Ajzen, I. (1975). Belief, attitude, intention, and behavior: An introduction to theory and research. Reading, MA: Addison-Wesley.

Fiske, A. P. (1992). The four elementary forms of sociality: Framework for a unified theory of social relations. Psychological Review, 99, 689-723.

Fiske, A., Kitayama, S., Markus, H. R., \& Nisbett, R. E. (1998). The cultural matrix of social psychology. In D. Gilbert, S. Fiske, \& G. Lindzey, The Handbook of Social Psychology (pp. 915-981). San Francisco, CA: McGraw-Hill.

Fiske, S. (2004). Social beings: Core motives in social psychology. New York: Wiley.

Fromme, K., Stroot, E., \& Kaplan, D. (1993). Comprehensive effects of alcohol: Development and psychometric assessment of a new expectancy questionnaire. Psychological Assessment, 5, 19-26. 
Frone, M. (1999). Developmental consequences of youth employment. In J. Barling, \& E. Kelloway, Young workers: Varieties of experience (pp. 89-128). Washington, D.C.: American Psychological Association.

Frone, M. (2003). Predictors of overall and on-the-job substance use among young workers. Journal of Occupational Health Psychology, 8, 39-54.

Gable, S., Reis, H., \& Elliot, A. J. (2000). Behavioral activation and inhibition in everyday life. Journal of Personality and Social Psychology, 78, 1135-1149.

Gecas, V. (1989). The social psychology of self-efficacy. Annual Review of Sociology, 291-316.

Goldman, M. S. (1994). The alcohol expectany concept: Applications to assessment, prevention, and treatment of alcohol abuse. Applied and Preventative Psychology, 3, 131-144.

Goldman, M., Del Boca, F., \& Darkes, J. (1999). Alcohol expectancy theory: The application of cognitive neuroscience. In K. Leonard, \& H. Blane, Psychological Theories of Drinking and Alcoholism (pp. 203-246). New York: Guilford Press.

Gong, Y., \& Fan, J. (2006). Longitudinal examination of the role of goal orientation in cross-cultural adjustment. Journal of Applied Psychology, 91, 176-184.

Green, A. S., Rafaeli, E., Bolger, N., Shrout, P. E., \& Reis, H. T. (2006). Paper or plastic? data equivalence in paper and electronic diaries. Psychological Methods, 11, 87-105. 
Greenfield, T. K., Bond, J., Roberts, S. C., Polcin, D., Korcha, R., Knibbe, R., et al. (2010, May). Alcohol harms, pressure to drink less and considering seeking help: A cross-national multilevel GENACIS analysis. Lausanne, Switzerland: 36th Annual Epidemiological Symposium of the Kettil Bruun Society for Social and Epidemiological Study of Alcohol.

Gresham, F. M., Evans, S., \& Elliott, S. N. (1988). Academic and social self-efficacy scale: Development and initial validation. Journal of Psychoeducational Assessment, 6, 125-138.

Grittner, U., Kuntsche, S., \& Bloomfield, K. (2010, May). Alcohol consumption and social inequality at individual and country level: Results from the GENACIS study. Lusanna, Switzerland: 36th Annual Alcohol Epidemiology Symposium of the Kettil Bruun Society for Social and Epidemiological Research on Alcohol.

Grube, J. W., Gruenewald, P. J., \& Chen, M. J. (2010). Community alcohol outlet density and underage drinking. Addiction, 105, 270-278.

Hasking, P. A., \& Oei, T. P. (2002). The differential role of alcohol expectancies, drinking refusal self-efficacy and coping resources in predicting alcohol consumption in community and clinical samples. Addiction Research and Theory, 10, 465-494.

Heine, S. J., Kitayama, S., Lehman, D. R., Takata, T., Ide, E., \& Leung, C. (2001). Divergent consequences of success and failure in Japan and North America: 
An investigation of self-improving motivations and malleable selves. Journal of Personality and Social Psychology, 81, 599-615.

Hermann, K., \& Betz, N. E. (2006). Path models of the relationships of instrumentality, social self-efficacy, and self-esteem to depression and loneliness. Journal of Social and Clinical Psychology, 25, 1086-1106.

Higuchi, S., \& Kono, H. (1994). Early diagnosis and treatment of alcoholism: The Japanese experience. Alcohol and Alcoholism, 29, 363-373.

Higuchi, S., Matsushita, S., \& Osaki, Y. (2006). Drinking practices, alcohol policiy and prevention programs in Japan, International Journal of Drug Policy, 102, 358-366.

Higuchi, S., Matsushita, S., Muramatsu, T., Murayama, M., \& Hayashida, M. (1996). Alcohol and aldehyde dehydrogenase genotypes and drinking behavior in Japanese, Journal of Clinical and Experimental Research, 20, 493-497.

Hirayama, F., Lee, A. H., Binns, C. W., Okumura, C., \& Yamamoto, S. (2009). Alcohol consumption by older adults in central and southern Japan. Asia Pacific Journal of Public Health, 21, 170-176.

Hoffman, D. (1998). Centering decisions in hierarchical linear models: Implications for research in organizations. Journal of Management, 24, 623-641.

Holtzman, M. T., \& DeLongis, A. (2007). One day at a time: The impact of daily spouse support on pain, negative affect, and catastrophizing among individuals with rheumatoid arthritis. Pain, 202-213. 
House, J. W., Umberson, D., \& Landis, K. R. (1988). Structures and processes of social support. Annual Review of Sociology, 14, 293-318.

Howell, D. C. (2002). Statistical methods for psychology (5th ed.). Pacific Grove, CA: Wadsworth.

Hox, J. (2002). Multilevel Analysis: Techniques and Applications. Mahwah, NJ. Institute of Medicine. (1998). Protecting youth at work. Washington, D.C.: National Academy Press.

Jerusalem, M., \& Schwarzer, R. (1992). Self-efficacy as a resource factor in stress appraisal processes. In R. Schwarzer, Self-efficacy: Thought control of action (pp. 195-213). Washington, D.C.: Hemisphere.

Johnson, C. N., \& Fromme, K. (1994). An experimental test of affect, subjective craving, and alcohol outcome expectancies as motivators of young adult drinking. Psychology of Addictive Behaviors, 19, 631-641.

Jones, E. (1998). Major developments in social psychology during the past five decades. In D. T. Gilbert, S. T. Fiske, \& G. Lindzey, Handbook of social psychology (4th ed., Vol. 1, pp. 3-57). Boston: McGraw-Hill.

Jones-Webb, R., Toomey, T., Miner, K., Wagenaar, A. C., Wolfson, M., \& Poon, R. (1997). Why and in what context adolescents obtain alcohol from adults: A pilot study. Substance Use and Misuse, 32, 219-228.

Judd, C. M., \& Kenny, D. (1981). Estimating the effects of social interventions. Cambridge: Cambridge University Press. 
Karasawa, M. (2001). A Japanese mode of self-making: Self-criticism and other enhancement. Japanese Journal of Psychology, 72, 198-209.

Kim, H., \& Markus, H. (1999). Deviance or uniqueness, harmony or conformity?: A cultural analysis. Journal of Personality and Social Psychology, 77, 785-800.

Kitano, H., Chi, I., Rhee, S., Law, C., \& Lubben, J. (1992). Norms and alcohol consumption: Japanese in Japan, Hawaii, and California, Journal of Studies on Alcohol, 22, 270-275.

Kitayama, S., \& Markus, H. R. (1999). Yin and yang of the Japanese self: The cultural psychology of personality coherence. In D. Cervone, \& Y. Shoda, The coherence of personality: Social cognitive bases of personality consistency, variability, and organization (pp. 242-302). New York: Guilford Press.

Kitayama, S., \& Markus, H. (2000). The pursuit of happiness and the realization of sympathy: Cultural patterns of self, social relations, and well-being. In E. Diener, \& E. M. Suhs, Cultural and Subjective Well-beign (pp. 113-161). Cambridge, MA: MIT Press.

Kitayama, S., \& Park, H. (2007). Cultural shaping of self, emotion and well-being: How does it work? Social and Personality Psychology Compass, 1, 202-222.

Kitayama, S., \& Uchida, Y. (2004). Interdependent agency: An alternative system for action. In R. Sorrentino, D. Cohen, J. M. Ison, \& M. P. Zanna, Culture and Social Behaviour: The Ontario Symposium (pp. 165-198). Mahwah, NJ: Lawrence Erlbaum Associates. 
Kitayama, S., Duffy, S., \& Uchida, U. (2007). Self as cultural mode of being. In S. Kitayama, \& D. Cohen, Handbook of Cultural Psychology (pp. 137-164). New York: Guilford Press.

Kitayama, S., Markus, H. R., \& Kurokawa, M. (2000). Culture, emotion, and wellbeing: Good feelings in Japan and the United States. Cognition and Emotion, $14,93-124$.

Kitayama, S., Markus, H., \& Matsumoto, H. (1995). Culture, self, and emotion: A cultural perspective on 'self-conscious' emotions. In J. P. Tangney, \& K. W. Fischer, Self-conscious emotions: The Psychology of Shame, Guilt, Embarassment, and Pride (pp. 439-464). New York: Guilford Press.

Kuntsche, E., Knibbe, R., Gmel, G., \& Engels, R. (2006). Who drinks and why? A review of socio-demographic, personality, and contextual issues behind the drinking motives in young people. Psychology of Addictive Behaviors, 31, 1844-1857.

Kwan, V. S., Bond, M. H., \& Singelis, T. M. (1997). Pancultural explanation for life satisfaction: Adding relationship harmony to self-esteem. Journal of Personality and Social Psychology, 73, 1038-1051.

Lazarus, R. S., \& Folkman, S. (1988). Transactional theory and research on emotions and coping. European Journal of Personality, 1, 141-169.

Lazarus, R., \& Cohen, J. (1977). Environmental stress. In I. Altman, \& J. Wohlwill, Human behavior and environment: Current theory and research (Vol. 2, pp. 89-128). New York: Plenum Press. 
Lebra, T. (1976). Japanese patterns of behavior. Honolulu, HI: University of Hawaii Press.

Lee, N., \& Oei, T. (1993). The importance of alcohol expectancies and drinking refusal self-efficacy in teh quantity and frequency of alcohol consumption. Journal of Substance Abuse, 5, 379-390.

Leigh, B. C. (1989). In search of the seven dwarves: Issues of measurement and meaning in alcohol expectancy research, Psychological Bulletin, 105, 361-373.

Leigh, B. C., \& Stacy, A. W. (1993). Alcohol outcome expectancies: Scale construction and predictive utility in higher order confirmatory models, Psychological Assessment, 5, 216-229.

Little, R., \& Rubin, D. (1987). Statistical analysis with missing data. New York: John Wiley.

Luczak, S. E., Glatt, S. J., \& Wall, T. L. (2006). Meta-analyses of ALDH2 and ADH1B and alcohol dependence in Asians: Examining models of influence, Psychological Bulletin, 132, 607-621.

MacCorquodale, K., \& Meehl, P. (1953). Preliminary suggestions as to a formalization of expectancy theory, Psychological Review, 60, 55-63.

Madson, L., \& Trafimow, D. (2001). Gender comparisons in the private, collective, and allocentric selves. Journal of Social Psychology, 141, 551-559.

Markus, H. R., \& Kitayama, S. (1991). Culture and the self: Implications for cognition, emotion, and motivation. Psychological Review, 98, 224-253. 
Markus, H. R., Kitayama, S., \& Heiman, R. (1997). Culture and "basic" psychological principles. In E. T. Higgins, \& A. W. Kruglanski, Social psychology: Handbook of basic principles (pp. 857-913). New York: Guilford Press.

Markus, H. R., Uchida, Y., Omoregie, H., Townsend, S. S., \& Kitayama, S. (2006). Going for the gold: Models of agency in Japanese and American contexts. Psychological Science, 17, 103-112.

Marlatt, G. A., \& Rosenhow, D. (1980). Cognitive processes in alcohol use: Expectancy and the balanced placebo design. In N. Emilo, Advances in substance abuse: Behavioral and biological reserach (pp. 159-199). Greenwich, CT: Jai Press.

Mascolo, M., Fischer, K., \& Li, J. (2003). Dynamic development of component systems of emotions: Pride, shame, and guilt in China and the United States. In R. J. Davidson, K. Schere, \& H. H. Goldsmith, Handbook of affective science (pp. 375-408). Oxford, U.K.: Oxford University Press.

Matsushima, R., \& Shiomi, K. (2003). Social self-efficacy and interpersonal stress in adolescence. Social Behavior and Personality, 31, 323-332.

Mertler, C., \& Vannatta, R. A. (2005). Advanced and Multivariate Statistical Methods. California: Pyrczak Publishing.

Mesquita, B. (2001). Emotions in Collectivist and Individualist contexts. Journal of Personality and Social Psychology, 80, 68-74.

Mesquita, B., \& Albert, D. (2007). The cultural regulation of emotions. In J. Gross, The handbook of emotion regulation (pp. 486-503). New York: Guilford Press. 
Mesquita, B., \& Karasawa, M. (2002). Different emotional lives. Cognition and Emotion, 16, 127-141.

Mesquita, B., \& Karasawa, M. (2004). Self-conscious emotions as dynamic cultural processes, Psychological Inquiry, 15, 161-166.

Mesquita, B., \& Leu, J. (2007). The cultural psychology of emotion. In S. Kitayama, \& D. Cohen, Handbook of Cultural Psychology (pp. 734-759). New York: Guilford Press.

Mezulins, A.H., Abramson, L.Y., Hyde, J.S., \& Hankin, B.L. (2004). Is there a universal positivity bias in attributions? A meta-analytic review of individual, developmental, and cultural differences in self-serving attributional bias, Psychological Bulletin, 130, 711-747.

Miller, P., Smith, G., \& Goldman, M. (1990). Emergence of alcohol expectancies in childhood: A possible critical period. Journal of Studies on Alcohol, 51, 343349.

Milne, D. (2003). Japan grapples with alcoholism crisis. Psychiatric News, 38, 12.

Miyamoto, Y., \& Kitayama, S. (2002). Cultural variation in correspondence bias: The critical role of attitude diagnosticity of socially constrained behavior. Journal of Personality and Social Psychology, 83, 1239-1248.

Mohr, C. D., Armeli, S., Ohannessian, C. M., Tennen, H., Carney, A., Affleck, G., et al. (2003). Daily interpersonal experiences and distress: Are women more vulnerable? Journal of Social and Clinical Psychology, 22, 393-423. 
Mohr, C. D., Armeli, S., Tennen, H., Temple, M., Todd, M., Clark, J., et al. (2005). Moving beyond the key party: A daily process investigation of college student drinking motivations. Psychology of Addictive Behaviors, 34, 222-254.

Mohr, C., Brannan, D., Mohr, J., Armeli, S., \& Tennen, H. (2008). Evidence for positive mood buffering among college student drinkers. Personality and Social Psychology Bulletin, 34, 1249-259.

Morling, B., Kitayama, S., \& Miyamoto, Y. (2002). Cultural practices emphasize influence in the United States and adjustment in Japan. Personality and Social Psychology Bulletin, 28, 311-323.

Nagoshi, C., Nakata, T., Sasano, K., \& Wood, M. D. (1994). Alcohol norms, expectancies, and reasons for drinking and alcohol use in a U.S. versus Japanese college sample. Alcoholism: Clinical and Experimental Research, 18, 671-678.

Nakamura, K., Tanaka, A., \& Takano, T. (1993). The social cost of alcohol abuse in Japan. Journal of Studies on Alcohol, 54, 618-625.

Nakaya, N., Kikuchi, N., Shimazu, T., Ohmori, K., Kakizaki, M., Sone, T., et al. (2007). Alcohol consumption and suidcide mortality among Japanese men: the Ohsaki study. Alcohol, 41, 503-510.

National Institute on Alcohol Abuse and Alcoholism. (2007). NIH Publication Number 03-5340. National Institute on Alcohol Abuse and Alcoholism. Bethesda: National Institutes of Health. 
National Tax Agency. (2004). Report prepared for a special meeting on retails of alcoholic beverages. Tokyo: Japan.

Oei, T. P., \& Burrow, T. (2000). Alcohol expectancy and drinknig refusal selfefficacy: A test of specificity theory. Addictive Behaviors, 25, 499-507.

Oei, T. P., \& Morawska, A. (2004). A cognitive model of binge drinking: The influence of alcohol expectancies and drinking refusal self-efficacy. Addictive Behaviors, 29, 159-179.

Oei, T. P., \& Young, R. M. (1993). Grape expectations: The role of alcohol expectations in the understanding and teratment of problem drinking. International Journal of Psychology, 28, 337-364.

Oei, T. P., Hasking, P. A., \& Young, R. (2005). Drinking refusal self-efficacy questionnaire revised (DRSEQ-R): A new factor strucutre with confirmatory factor analysis. Drug and Alcohol Dependence, 78, 297-307.

Oei, T. P., Hasking, P., \& Phillips, L. (2007). A comparison of general self-efficacy and drinking refusal self-efficacy in predicting drinking behaviour. American Journal of Drug and Alcohol Abuse, 33, 833-841.

Oei, T., Fergusson, S., \& Lee, N. (1998). The differential role of alcohol expectancies and drinking refusal self-efficacy in problem and nonproblem drinkers. Journal of Studies on Alcohol, 59, 704-711.

Oishi, S., \& Diener, E. (2001). Goals, culture, and subjective well-being. Personality and Socialy Psychology Bulletin, 27, 1674-1682. 
O'Leary, A. (1992). Self-efficacy and health: Behavioral and stress-physiological mediation. Cognitive Therapy and Research, 16, 229-245.

O'Malley, P. M., \& Johnston, L. D. (2002). Epidemiology of alcohol and other drug use among American college students. Journal of Studies on Alcohol, 14, 2339.

Osaki, Y., Matsushita, S., Shirasaka, T., Hiro, H., \& Higuchi, S. (2005). Nationwide survey of alcohol drinking and alcoholism among Japanese adults. Japanese Journal of Alcohol and Drug Dependence, 40, 455-470.

Osaki, Y., Tanihata, T., Ohida, T., Kanda, H., Suzuki, K., Higuchi, S., et al. (2008). Decrese in the prevalence of adolescent alcohol use and its possible causes in Japan: Periodical nationwide cross-sectional surveys. Alcohol: Clinical and Experimental Research, 33, 247-254.

Parrish, K. M., Higuchi, S., \& Lucas, L. J. (1993). Increased alcohol-relatd esophageal cancer mortality rates in Japanese men. International Journal of Epidemiology, 22, 600-605.

Parrish, K. M., Higuchi, S., Muramatsu, T., Stinson, F. S., \& Harford, T. C. (1991). A method for estimating alcohol-related cirrhosis mortality in Japan. International Journal of Epidemiology, 20, 921-926.

Perkins, H. (2002). Surveying the damage: A reivew of research on consequences of alcohol misuse in college populations. Journal of Studies on Alcohol, Supplement 14, 91-100. 
Perkins, H. W., \& Berkowitz, A. D. (1986). Perceiving the community norms of alcohol use among students: Some research implications for campus alcohol education programming. International Journal of Addictions, 21, 961-976.

Perrine, M. W., Mundt, J. C., Searles, J. S., \& Lester, L. S. (1995). Validation of daily self-report alcohol consumption using interaction voice response (IVR) technology. Journal of Studies on Alcohol, 56, 487-490.

Perry, D. G., Perry, L. C., \& Rasmussen, P. (1986). Cognitive and social learning mediators of agression. Child Development, 45, 55-62.

Pittman, D. J. (1967). International overview: Social and cultural factors in drinking patterns, pathological and nonpathological. In D. J. Pittman, Alcoholism (pp. 320). New York: Harper \& Row.

Raudenbush, S. W., Bryk, A. S., Cheong, Y. F., \& Congdon, R. T. (2000). HLM 5: Hierarchical linear and nonlinar modeling. Chicago: Scientific Software International.

Reis, H. (1994). Domains of experience: Investigating relationship processes from three perspectives. In R. Erber, \& R. Gilmour, Theoretical frameworks for personal relationships (pp. 87-110). Hillsdale, NJ: Lawrence Erlbaum Associates, Inc.

Reis, H., \& Gable, S. (2000). Event-sampling and other methods for studying everyday experience. In H. Reis, \& C. Judd, Handbook of Research Methods in Social and Personality Psychology (pp. 190-222). New York: Cambridge University Press. 
Reis, H., \& Gable, S. (2003). Toward a positive psychology of relationships. In C. L. Keyes, \& J. Haidt, Flourishing: The positive person and the good life (pp. 129159). Washington, DC: American Psychological Association.

Robinson, M. D., \& Clore, G. L. (2002). Belief and feeling: Evidence for an accessibility model of emotional self-report. Pychological Bulletin, 128, 934960.

Robinson, M. D., \& Clore, G. L. (2002). Episodic and semantic knowledge in emotional self-report: Evidence for two judgement processes. Journal of Personality and Social Psychology, 83, 198-215.

Rohsenow, D. J. (1983). Social anxiety, daily moods, and alcohol use over time among heavy social drinking men. Journal of Addictive Behaviors, 7, 311-315.

Rosenstock, I. M. (1966). Why people use health services. Milbank Memorial Fund Quarterly, 44, 94-127.

Sadakane, A., Gotoh, T., Ishikawa, S., Nakamura, Y., \& Kayaba, K. (2009). Amount and frequency of alcohol consumption and all-cause mortality in a Japanese population: The JMS cohort study. Journal of Epidemiology, 19, 107-115.

Salovey, P., Rothman, A., \& Rodin, J. (1998). Health behavior. In D. Gilbert, S. Fiske, \& G. Lindzey, The handbook of social psychology (4th ed., Vol. 2, pp. 633683). New York: McGraw-Hill.

Saltzman, K., \& Holahan, C. (2002). Social support, self-efficacy, and depressive symptoms: An integrative model. Journal of Social and Clinical Psychology, 21, 309-322. 
Sampson, E. (1988). The debate on individualism: Indigenous psychologies of the individual and their role in personal and societal functioning. American Psychologist, 43, 15-22.

Scollon, C., Diener, E., Oishi, S., \& Biswas-Diener, R. (2004). Emotions across cultures and methods. Journal of Cross-Cultural Psychology, 35, 304-326.

Sears, D. O. (1987). College sophomores in the laboratory: Influences of a narrow database on social psychology's view of human nature. Journal of Personality and Social Psychology, 51, 515-530.

Shadish, W., Cook, T., \& Campbell, D. (2002). Experimental and quasi-experimental designs for generalized causal inference. Boston: Houghton Mifflin.

Sher, K. J., Wood, M. D., Wood, P. K., \& Raskin, G. (1996). Alcohol outcome expectancies and alcohol use: A latent variable cross-lagged panel study. Journal of Abnormal Psychology, 105, 561-574.

Shiffman, S. (2000). Real-time self-report of momentary states in the natural environment: Computerized ecological momentary assessment. In A. A. Stone, J. S. Turkkan, C. A. Bachrach, J. B. Jobe, H. S. Kurtzman, \& V. S. Cain, The science of self-report: Implications for research and practice (pp. 277-296). Mahwah, NJ: Lawrence Erlbaum Associates Publishers.

Shimizu, H. (2000). Japanese culturla psychology and empathic understanding: Implications for academic and cultural psychology. Ethos, 28, 224-247.

Shimizu, S. (1990). An alcoholic social system: Drinking culutre and drinking behaviors in Japan. Japanese Journal of Mental Health, 36, 85-100. 
Smith, G., Goldman, M. S., Greenbaum, P., \& Christiansen, B. (1995). Expectancy for social facilitation from drinking: The divergent paths of high-expectancy and low-expectancy adolescents. Journal of Abnormal Psychology, 104, 32-40.

Smith, H., \& Betz, N. (2000). Development and evaluation of a measure of social selfefficacy in college students. Journal of Career Assessment, 56, 35-52.

Smith, H., \& Betz, N. E. (2002). Examination of efficacy and esteem pathways to depression. Journal of Counseling Psychology, 49, 438-448.

Snijders, T., \& Bosker, R. (1999). Multilevel analysis: An introduction to basic and advanced multilevel modeling. Thousand Oaks, CA: Sage Publications.

Snyder, M., \& White, P. (1982). Moods and memories: Elation, depression, and the remembering of the events of one's life. Journal of Personality, 50, 149-167.

Stacy, A. W., Widaman, K. F., \& Marlatt, A. G. (1990). Expectancy models of alcohol use. Journal of Personality and Social Psychology, 58, 918-928.

Stern, P., Dietz, T., Abel, T., Guagnano, G. A., \& Kalof, L. (1999). A value-beliefnorm theory of support for social movements: The case of environmental concern. Human Ecology Review, 6, 81-97.

Stone, A. A., \& Shiffman, S. (1994). Ecological momentary assessment (EMA) in behavioral medicine. Annals of Behavioral Medicine, 16, 199-202.

Stone, A. A., Shiffman, S. S., \& DeVries, M. W. (1999). Ecological momentary assessment. In D. Kahneman, E. Diener, \& N. Schwarz, Well-being: The foundations of hedonic psychology (pp. 26-29). New York: Russell Sage Foundation. 
Stroebe, W., \& Stroebe, M. (1996). The social psychology of Social support. In Social psychology: Handbook of basic principles. Guilford Publications, Inc.

Suh, E. M., Diener, E., Oishi, S., \& Triandis, H. C. (1998). The shifting basis of life satisfaction judgments across cultures: Emotions versus norms. Journal of Personality and Social Psychology, 74, 484-493.

Tabachnick, B. G., \& Fidell, L. S. (1996). Using Multivariate Statistics (3rd ed.). New York: Harper Collins.

Takano, T., Nakamura, K., \& Watanabe, M. (1996). Increased female drinking in accoradance with pos-industrial urbanization in Japan. Alcohol and Alcoholism, 31, 41-49.

Takeshita, T., \& Morimoto, K. (1999). Self-reported alcohol-associated symptoms and drinking behavior in three ALDH2 genotypes among Japanese university students. Alcohol: Clinical and Experimental Research, 23, 1065-1069.

Tennen, H., \& Affleck, G. (1996). Daily process in coping with chronic pain: Methods and analytic strategies. In M. Zeidner, \& N. Endler, Handbook of Coping: Theory, Research, Applications (pp. 151-177). New York: Wiley.

Tennen, H., Affleck, G., Armeli, S., \& Carney, M. A. (2000). A daily process approach to coping: Linking theory, research, and practice. American Psychologist, 55, 626-636.

Tennen, H., Affleck, G., Coyne, J. C., Larsen, R. J., \& DeLongis, A. (2006). Paper and plastic in daily diary research. Psychological Methods, 11, 112-118. 
Tennen, H., Suls, J., \& Affleck, G. (1991). Personality and daily expeirence: The promise and the challenge. Journal of Personality (Special Issue), 59, 313-337.

Thavorncharoensap, M., Teerawattananon, Y., Yothasamut, J., Lertpitakpong, C., \& Chaikledkaew, U. (2009). The economic impact of alcohol consumption: A systematic review. Substance Abuse Treatment, Prevention, and Policy, 4.

Thomas, D. L., \& Diener, E. (1990). Memory accuracy in the recall of emotions. Journal of Personality and Social Psychology, 59, 291-297.

Thomas, D., \& Diener, E. (1990). Memory accuracy in the recall of emtions. Journal of Personality and Social Psychology, 59, 291-297.

Triandis, H. (1989). The self and social behavior in differing cultural contexts. Psychological Review, 96, 506-520.

Uchida, Y., \& Kitayama, S. (2009). Happiness and unhappiness in east and west: Themes and variations. Journal of Emotion, 9, 441-456.

Uehata, T., Suzuki, K., Wada, K., Yamaguchi, N., Minowa, M., \& Oida, T. (2001). Report on the 2000 nationla survey on adolescent drinking behavior. Tokyo: Japan: Study Group for the 2000 National Survey on Adolescent Drinking Behavior.

Vieno, A., Santinello, M., Pastore, M., \& Perkins, D. (2007). Social support, sense of community in school, and self-efficacy as resources during early adolescence: An integrative model. American Journal of Community Psychology, 39, 177190. 
Wada, K., Price, R. K., \& Fukui, S. (1998). Reflecting adult drinking culture: Prevalence of alcohol use and drinking situations among Japanese junior high school students in Japan. Journal of Studies on Alcohol, 59, 381-386.

Wang, M., Liu, S., Zhan, Y., \& Shi, J. (2010). Daily work-family conflict and alcohol use: Testing the cross-level moderation effects of peer drinking norms and social support, Journal of Applied Psychology, 95, 377-386.

Wechsler, H., Nelson, T. F., Lee, J. E., Seibring, M., Lewis, C., \& Keeling, R. P. (2002). Perception and reality: A national evaluation of social norms marketing interventions to reduce college students' heavy alcohol use. Quarterly Journal of Studies on Alcohol, 64, 484-494.

West, S. G., \& Hepworth, J. T. (1991). Statistical issues in the study of temporal data: Daily experiences. Journal of Personality, 59, 609-662.

Wheeler, L., \& Reis, H. (1991). Self-recording of events in everyday life: Origins, types, and uses. Journal of Personality , 59 (3), 339-354.

Wie, M., Russell, D. W., \& Zakalik, R. A. (2005). Adult attachment, social selfefficacy, sefl-disclosure, loneliness, and subsequent depression for freshmen college students: A longitudinal study. Journal of Counseling Psychology, 52, 602-614.

Wigfield, A. (1994). Expectancy-value theory of achievement motivation: A developmental perspective. Educational Psychology Review , 6 (1), 49-78.

Wortman, C. B., Abbey, A., Holland, A. E., Silver, R. L., \& Janoff-Bulman, R. (1980). Transitions from the laboratory to the field: Problems and progress. In 
L. Bickman, Applied social psychology annual (pp. 197-233). Beverly Hills, CA: Sage.

Xie, D. (2007). Buffering or strengthening: The moderating effect of self-efficacy on stressor-strain relationship. Journal of Career Assessment , 15 (3), 351-366.

Yeh, C., Inose, M., Kobori, A., \& Chang, T. (2001). Self and coping among college students in Japan. Journal of College Student Development , 42 (3), 242-256.

Young, R. M., Connor, J. P., Ricciardelli, L. A., \& Saunders, J. B. (2006). The role of alcohol expectancy and drinking refusal self-efficacy beliefs in university student drinking. Alcohol and Alcoholism , 41 (1), 70-75. 
Appendix A. Initial Assessment Measures

\section{Social Self-Efficacy (8-items)}

Directions: Please read each statement carefully. Then decide how much confidence you have that you could perform each of these activities successfully. Please use the following scale to rate your confidence, and circle the number corresponding to your confidence level after each activity.

1-No Confidence at all

2-Little Confidence

3-Moderate Confidence

4-Much Confidence

5-Complete Confidence

How much confidence do you have that you could

1. Start a conversation with someone you don't know very well

2. Put yourself in a new and different social situation

3. Ask a group of people who are planning to engage in a social activity (e.g., go to a movie) if you can join

4. Get invited to a party that is being given by a prominent or popular individual

5. Go to a party or social function where you probably won't know anyone

6. Join a lunch or dinner table where people are already sitting and talking

7. Make friends in a group where everyone else knows each other

8. Ask someone out after he/she was busy the first time you asked

\section{Drinking Refusal Self-Efficacy (19-items)}

Please indicate how sure or unsure you are that you would drink in the following situations:

$1=\mathrm{I}$ am very sure I would drink

$2=I$ am somewhat sure I would drink

$3=\mathrm{I}$ am a little sure I would drink

4=I am a little sure I would not drink

$5=$ I am somewhat sure I would not drink

$6=\mathrm{I}$ am very sure I would not drink

Questions:

1. When I am out to dinner

2. When someone offers me a drink

3 . When my spouse or partner is drinking

4. When my friends are drinking 
5. When I am at a pub or a club

6. When I am angry

7. When I am frustrated

8. When I am worried

9. When I feel upset

10. When I feel down

11. When I feel nervous

12. When I feel sad

13. When I am watching TV

14. When I am at lunch

15. When I am on the way home from work

16. When I am listening to music or reading

17. When I am by myself

18. When I have just finished playing sports

19. When I first arrive home 
Appendix B. Daily interview measures

\section{Alcohol consumption last night (6-Items)}

1. How many alcoholic drinks did you have AT HOME (e.g., in your apartment or dorm room) last night?

$0123456789101112131415>15$

2. When drinking at HOME last night, how many drinks did you have with others?

$0123456789101112131415>15$

3. When drinking at HOME last night, how many drinks did you have alone?

$0123456789101112131415>15$

4. How many alcoholic drinks did you have AWAY FROM HOME (e.g., at bar, friend's apartment or dorm room) last night?

$0123456789101112131415>15$

5. When drinking AWAY FROM HOME last night, how many drinks did you have with others?

$0123456789101112131415>15$

6. When drinking AWAY FROM HOME last night, how many drinks did you have alone?

$0123456789101112131415>15$

\section{Daily Social Events (13-items)}

Below you will find a list of different types of events that may or may not have occurred to you TODAY. Please indicate whether or not each event occurred TODAY. If yes, please click on the appropriate rating.

\section{SOCIAL Occurred? Extremely Extremely Undesirable Desirable}

Spent pleasant or relaxing time with friend(s) or

$\begin{array}{lllllllllll}\text { boyfriend/girlfriend. } & \text { YES } & \text { NO } & 1 & 2 & 3 & 4 & 5 & 6 & 7\end{array}$

Had a disagreement or conflict with friend(s) or

$\begin{array}{lllllllllll}\text { boyfriend/girlfriend } & \text { YES } & \text { NO } & 1 & 2 & 3 & 4 & 5 & 6 & 7\end{array}$

Received a compliment on my physical appearance.

$\begin{array}{lllllllll}\text { YES NO } & 1 & 2 & 3 & 4 & 5 & 6 & 7\end{array}$


Something happened that made me feel awkward or

embarrassed in public

YES NO

Did something special for friend(s) or boyfriend/

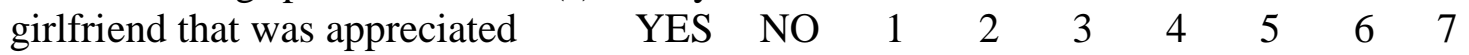

Had especially good interactions with friend(s),

$\begin{array}{llllllll}\text { Boyfriend/girlfriend or acquaintance(s).YES NO } & 1 & 2 & 3 & 4 & 5 & 6 & 7\end{array}$

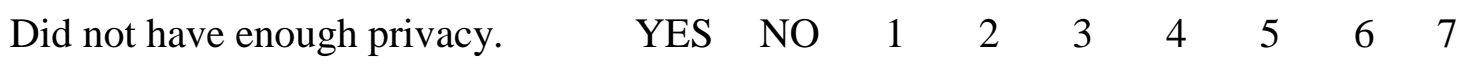

Had a an unpleasant interaction with someone other than a friend, boyfriend/girlfriend or family member.

$\begin{array}{lllllllll}\text { YES } & \text { NO } & 1 & 2 & 3 & 4 & 5 & 6 & 7\end{array}$

Sent or received an enjoyable letter/e-mail/phone call

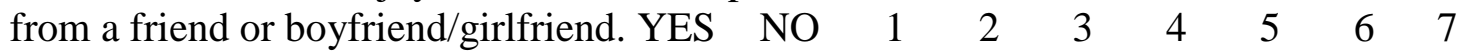

Went out socializing with friend(s) or boyfriend/

$\begin{array}{llllllllll}\text { girlfriend (e.g., party, club). } & \text { YES } & \text { NO } & 1 & 2 & 3 & 4 & 5 & 6 & 7\end{array}$

Friends were not available when I wanted to

$\begin{array}{llllllllll}\text { socialize. } & \text { YES } & \text { NO } & 1 & 2 & 3 & 4 & 5 & 6 & 7\end{array}$

Others did not do something that I wanted them to

$\begin{array}{llllllllll}\text { do. } & \text { YES } & \text { NO } & 1 & 2 & 3 & 4 & 5 & 6 & 7\end{array}$

$\begin{array}{lllllllll}\text { Provided support for someone I care for. YES NO } & 1 & 2 & 3 & 4 & 5 & 6 & 7\end{array}$

\section{Alcohol Outcome Expectancy Desirability (9-items)}

Please rate how DESIRABLE or UNDESIRABLE you think the following experiences would be TONIGHT, by clicking on the appropriate rating.

Not at all Desirable Very Desirable

1. A sense of accomplishment from studying.

$\begin{array}{lllllll}1 & 2 & 3 & 4 & 5 & 6 & 7\end{array}$

2. Reduced tension from drinking alcohol.

$\begin{array}{lllllll}1 & 2 & 3 & 4 & 5 & 6 & 7\end{array}$

3. A sense of accomplishment from exercise/physical

$\begin{array}{lllllll}1 & 2 & 3 & 4 & 5 & 6 & 7\end{array}$ activity

4. Physical impairment (becoming clumsy or

1223455667 uncoordinated) from drinking alcohol.

5. Losing myself in TV or a movie.

$\begin{array}{lllllll}1 & 2 & 3 & 4 & 5 & 6 & 7\end{array}$

6. A sense of carelessness from drinking alcohol.

$\begin{array}{lllllll}123 & 4 & 5 & 6 & 7\end{array}$ 
7. Losing myself in video/computer games.

$\begin{array}{lllllll}1 & 2 & 3 & 4 & 5 & 6 & 7\end{array}$

8. A feeling of closeness from hanging out with friends.

$\begin{array}{lllllll}1 & 2 & 3 & 4 & 5 & 6 & 7\end{array}$

9. A pleasant feeling from drinking alcohol with friends.

$\begin{array}{lllllll}1 & 2 & 3 & 4 & 5 & 6 & 7\end{array}$

